\title{
Twenty-Vertex model with Domain Wall Boundaries and Domino tilings
}

\author{
Philippe Di Francesco ${ }^{1,2} * \quad$ Emmanuel Guitter ${ }^{2} \dagger$ \\ 1. Department of Mathematics \\ University of Illinois \\ Urbana, IL 61821, U.S.A.
}

2. Université Paris Saclay, CNRS, CEA

Institut de physique théorique

91191 Gif-sur-Yvette, France

philippe@illinois.edu emmanuel.guitter@ipht.fr

Submitted: Jun 20, 2019; Accepted: Mar 28, 2020; Published: May 1, 2020

(C) The authors. Released under the CC BY-ND license (International 4.0).

\begin{abstract}
We consider the triangular lattice ice model (20-Vertex model) with four types of domain-wall type boundary conditions. In types 1 and 2, the configurations are shown to be equinumerous to the quarter-turn symmetric domino tilings of an Azteclike holey square, with a central cross-shaped hole. The proof of this statement makes extensive use of integrability and of a connection to the 6-Vertex model. The type 3 configurations are conjectured to be in same number as domino tilings of a particular triangle. The four enumeration problems are reformulated in terms of four types of Alternating Phase Matrices with entries 0 and sixth roots of unity, subject to suitable alternation conditions. Our result is a generalization of the ASM-DPP correspondence. Several refined versions of the above correspondences are also discussed.
\end{abstract}

Mathematics Subject Classifications: 05B45, 05A15, 82B20, 82B23

\section{Introduction}

Few combinatorial objects have fostered as many and as interesting developments as Alternating Sign Matrices (ASM). As described in the beautiful saga told by Bressoud [Bre99], these had a purely combinatorial life of their own from their discovery in the 80's

\footnotetext{
*Partially supported by the Morris and Gertrude Fine endowment, the NSF grant DMS18-02044 and the Simons Fellowship No 617036.

${ }^{\dagger}$ Supported by the grant ANR-14-CE25-0014 (ANR GRAAL)
} 
by Mills, Robbins and Rumsey [MRR83] to their enumeration [Zei96a, Zei96b] in relation to other intriguing combinatorial objects such as plane partitions with maximal symmetries [MRR86] or descending plane partitions (DPP)[And80, Lal03, Kra06, BDFZJ12, BDFZJ13], until they crashed against the tip of the iceberg of two-dimensional integrable lattice models of statistical physics [Bax89]. This allowed not only for an elegant proof of the so-called ASM conjecture [Kup96] and its variations by changing its symmetry classes [Kup02], but set the stage for future developments, with some new input from physics of the underlying six-vertex $(6 \mathrm{~V})$ "ice" model in the presence of special domain wall boundary conditions (DWBC), and its relations to a fully-packed loop gas, giving eventually rise to the Razumov-Stroganov conjecture [RS04], finally proved by Cantini and Sportiello in 2010 [CS11].

The ice model involves configurations on a domain of square lattice, obtained by orienting each individual edge in such a way that the ice rule is obeyed at each node, namely that exactly two edges point in and two edges point out of the node. It is now recognized that the statistical model for two-dimensional ice, solved in [GAL72] is at the crossroads of many combinatorial wonders, in relation with loop gases, osculating paths, rhombus and domino tiling problems, and even equivariant cohomology of the nilpotent matrix variety. Moreover, due to its inherent integrable structure, the model offers a panel of powerful techniques for solving, such as transfer matrix techniques, the various available Bethe Ansätze, Izergin and Korepin's determinant, the quantum Knikhnik-Zamolodchikov equation, etc. [GAL72, Bax89, ICK92, Che92, DFZJ05a, DFZJ05b].

In the present paper, we develop and study the combinatorics of the ice model on the regular triangular lattice, known as the Twenty-vertex (20V) model [Kel74, Bax89]. Focussing on the combinatorial content, we introduce particular "square" domains $(n \times$ $n$ rhombi of the triangular lattice) with special boundary conditions meant to create domain walls, i.e. separations between domains of opposite directions of oriented edges, in an attempt to imitate the $6 \mathrm{~V}$ situation. Among the many possibilities offered by the triangular lattice geometry, we found two particularly interesting classes of models, which we refer to as 20V-DWBC1,2 (where DWBC1 and DWBC2 are two flavors of the same class viewed from different perspectives) and 20V-DWBC3. These are respectively enumerated by the sequences:

$$
\begin{aligned}
& A_{n}=1,3,23,433,19705,2151843,561696335,349667866305 \ldots(\mathrm{DWBC} 1,2) \\
& B_{n}=1,3,29,901,89893,28793575 \ldots(\mathrm{DWBC} 3)
\end{aligned}
$$

for $n=1,2, \ldots$

From their definition, both models can be interpreted as generalizations of ASMs, in which non-zero entries may now belong to the set of sixth roots of unity, and we shall refer to them as Alternating Phase Matrices (APM) of types 1,2,3 respectively. To further study both sets of objects, we use the integrable version of the 20V model [Kel74, Bax89] to (i) decorate the model's configurations with Boltzmann weights parameterized by complex spectral parameters; and (ii) reformulate whenever possible the partition function. In this paper, we succeed in performing this program in the case of 20V-DWBC1,2, which is eventually reformulated as an ordinary $6 \mathrm{~V}$-DWBC model on a square grid, but with 
non-trivial Boltzmann weights $(a, b, c)=(1, \sqrt{2}, 1)$. The case of DWBC3 is more subtle as the model can be rephrased as a $6 \mathrm{~V}$ model with staggered boundary conditions.

Among other possibilities, the $20 \mathrm{~V}$ configurations may be represented as configurations of some non-intersecting paths with steps along the edges of the triangular lattice, with the possibility of double or triple kissing (osculation) points at vertices. Individually, the same paths, once drawn on a straightened triangular lattice equal to the square lattice supplemented with a second diagonal edge on each face, are nothing but the Schröder paths on $\mathbb{Z}^{2}$, with horizontal, vertical and diagonal steps $(1,0),(0,-1)$ and $(1,-1)$. These are intimately related to problems of tiling of domains of the square lattice by means of $1 \times$ 2 and $2 \times 1$ dominos, such as the celebrated Aztec diamond domino tiling problem [CEP96], or the more recently considered Aztec rectangles with boundary defects [BK18, DFG19].

Looking for candidates in the domino tiling world for being enumerated by the sequences $A_{n}$ or $B_{n}$, we found the two following remarkable models.

In the case of $A_{n}$, the associated domino tiling problem is a natural generalization of the descending plane partitions introduced by Andrews [And80] and reformulated as the rhombus tiling model of a hexagon with a central triangular hole, with $2 \pi / 3$ rotational symmetry [Lal03, Kra06], which allows to interpret it also as rhombus tilings of a cone. In the same spirit, we find that $A_{n}$ counts the domino tiling configurations of an Aztec-like, quasi-square domain, with a cross-shaped central hole, and with $\pi / 2$ rotational symmetry, or equivalently the domino tilings of a cone. The fact that $20 \mathrm{~V} \mathrm{DWBC} 1,2$ configurations and quarter-turn symmetric domino tilings of a holey square are enumerated by the sane sequence $A_{n}$ is proved in the present paper, together with a refinement, which parallels the refinement in the so-called ASM-DPP conjecture of Mills, Robbins and Rumsey [MRR83]. We use similar techniques to those of [BDFZJ12, BDFZJ13], namely identify both counting problems as given by determinants of finite truncations of infinite matrices, whose generating functions are simply related.

In the case of $B_{n}$, a natural candidate was found by using the online encyclopedia of integer sequences (OEIS). The number of domino tilings of a square of size $2 n \times$ $2 n$ is $2^{n} b_{n}^{2}$ [Kas63, $\left.\mathrm{FMP}^{+} 15\right]$, where $b_{n}$ itself counts the number of domino tilings of a triangle obtained by splitting the square into two equal domains [Pac97]. We found perfect agreement between our data for $B_{n}$ and $b_{n}$, which we conjecture to be equal for all $n$. Despite many interesting properties of the model, we have not been able to prove this correspondence.

The paper is organized as follows.

In Section 2, we introduce the 20V model and define a first class of models in its two flavors of domain wall boundary conditions DWBC1,2, conveniently expressed in terms of osculating Schröder paths. We also define the special integrable weights, parameterized by triples of complex spectral parameters $z, t, w$, and obeying the celebrated Yang-Baxter relation.

These models are mapped onto the $6 \mathrm{~V}$-DWBC model (Theorem 1) with anisotropy parameter $\Delta=1 / \sqrt{2}$ in Section 3, by use of the integrability property, leading to compact formulas for the partition function and in particular the numbers $A_{n}$, in the form of some simple determinant, with known asymptotics leading to the free energy $f=\frac{3}{2} \log \frac{4}{3}$ per 
site. The integrability of the model allows us moreover to keep track of a refinement of the number $A_{n}$ according to the number of occupied vertical edges in the last column of the domain, in the osculating Schröder path formulation (Theorem 2). The generating polynomial of the refined numbers is also given by some simple determinant.

Section 4 is devoted to the definition and enumeration of the domino tiling problem of a cone corresponding to the sequence $A_{n}$, which generalizes the notion of Andrews' descending plane partitions in domino terms. After defining the tiling problem, we perform enumeration using a non-intersecting Schröder path formulation and Gessel-Viennot determinants (Theorem 4). We also introduce refinements in the same spirit as the refinements of the DPP conjecture of [MRR83] (Theorem 5).

The equivalence between the enumerations in Sections 3 and 4 is proved in Section 5 , in the same spirit as the refined ASM-DPP proof of [BDFZJ12]. We first evaluate the homogeneous limit of the Izergin-Korepin determinantal formula for the $6 \mathrm{~V}$-DWBC partition function, and write it in the form of the determinant of the finite $n \times n$ truncation of an infinite matrix independent of $n$. We then identify this determinant with the GesselViennot determinant of Section 4 (Theorem 7). We also work out the refined version of this result, by keeping one non-trivial spectral parameter in the $20 \mathrm{~V}$ model, and identifying it in a special weighting of the Schröder path configurations for the domino tiling of the cone of Section 4 (Theorem 8).

We turn to other possible domain wall boundary conditions in Section 6. We first define the 20V-DWBC3 model and formulate the Conjecture 10 that its configurations are also enumerated by the domino tilings of the triangle of [Pac97]. We extend this to a sequence of models corresponding to half-hexagonal shapes with the same boundary conditions, conjecturally enumerated by the domino tilings of Aztec-like extensions of the former triangle (Conjecture 11). We complete the section with another possible DWBC4 for which no general conjecture was formulated.

In Section 7, we identify the various 20V-DWBC configurations considered in this paper with sets of matrices with entries made of triples of elements in $\{0,1,-1\}$, or equivalently taking values among 0 and the sixth roots of unity, that generalize the notion of Alternating Sign Matrix.

We gather a few concluding remarks in Section 8.

\section{The 20V model with Domain Wall Boundary Conditions}

\subsection{Definition of the model: ice rule and osculating paths}

The combinatorial problem that we wish to address corresponds to a particular instance of the $20 \mathrm{~V}$ model on a finite regular domain with specific boundary conditions. The corresponding geometry is directly inspired from that of the $6 \mathrm{~V}$ model on a portion of square lattice with Domain Wall Boundary Conditions (DWBC) [Kor82], suitably adapted to the triangular lattice as follows: we first straighten the triangular lattice into a square lattice supplemented with a second diagonal within each face. In this setting, the regular 

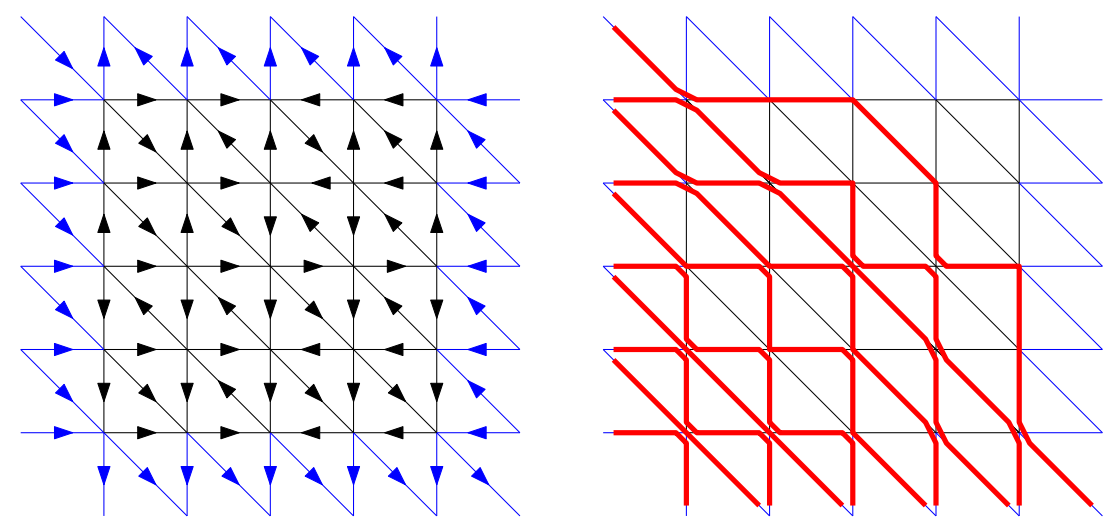

Figure 1: Left: A sample configuration of a 20V model configuration with DWBC1. Right: The equivalent osculating path configuration.
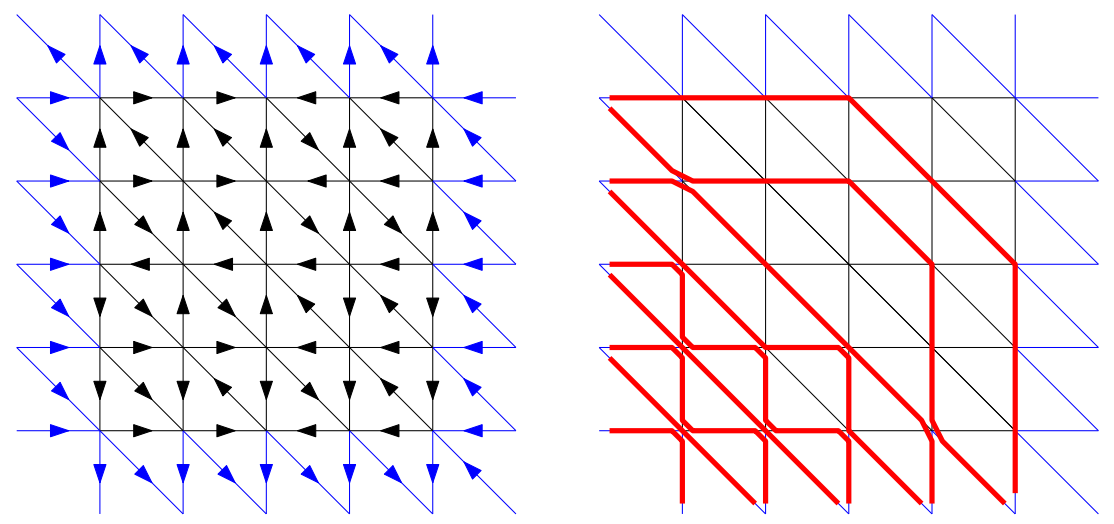

Figure 2: Left: A sample configuration of a $20 \mathrm{~V}$ model configuration with DWBC2. The boundary condition differs from that of Fig. 1 by the orientation imposed on the upper-left and lower-right edges. Right: The equivalent osculating path configuration.

domain underlying our $20 \mathrm{~V}$ model is an $n \times n$ square portion of lattice ${ }^{1}$, whose vertices occupy all points with integer coordinates $(i, j)$ for $i, j=1,2, \ldots, n$. Its set of inner edges is made of all the elementary horizontal segments $(i, j) \rightarrow(i+1, j)(i<n)$ and vertical segments $(i, j) \rightarrow(i, j+1)(j<n)$ joining neighboring vertices, as well as all the second diagonals $(i, j+1) \rightarrow(i+1, j)(i, j<n)$. This edge set is completed by a set of oriented boundary edges, with the following prescribed orientations:

- West boundary: horizontal edges oriented from $(0, j)$ to $(1, j), j=1,2, \ldots, n$ and diagonal edges oriented from $(0, j+1)$ to $(1, j)$ for $j=1,2, \ldots n-1$;

- South boundary: vertical edges oriented from $(i, 1)$ to $(i, 0), i=1,2, \ldots, n$ and diagonal edges oriented from $(i, 1)$ to $(i+1,0)$ for $i=1,2, \ldots n-1$;

\footnotetext{
${ }^{1}$ Before straightening, this domain is an $n \times n$ rhombus of the triangular lattice.
} 


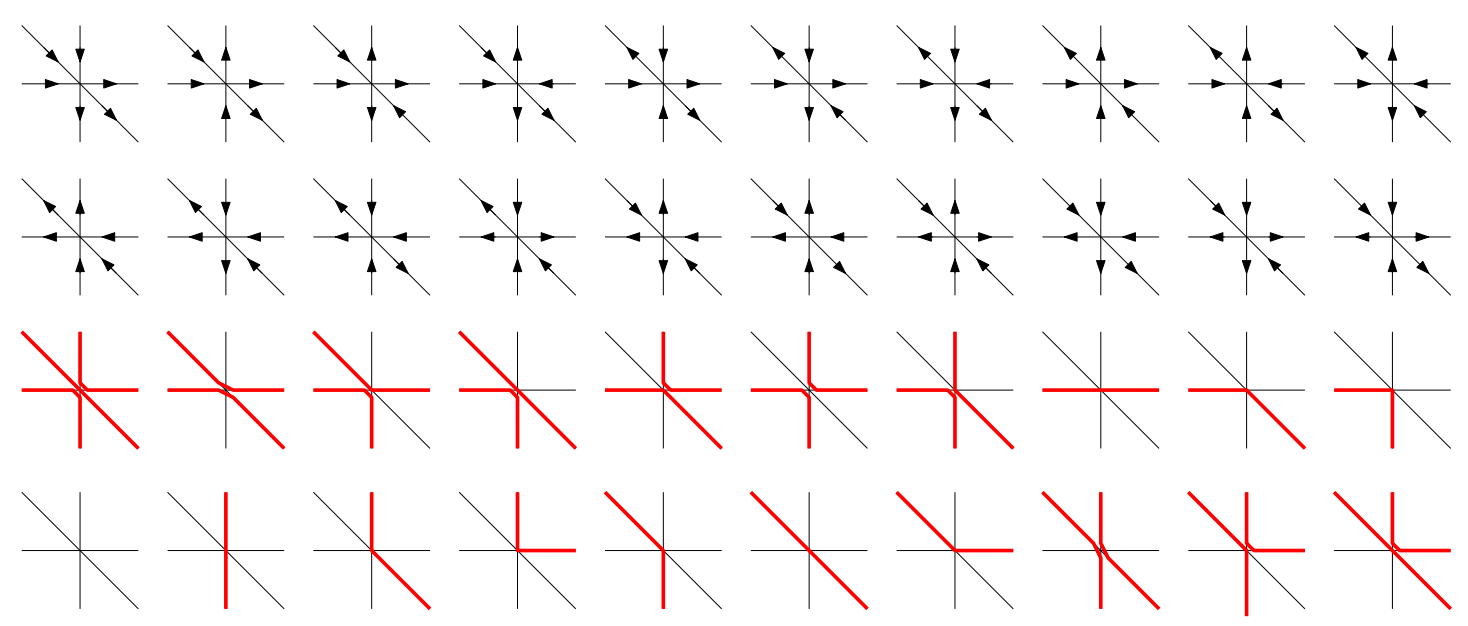

Figure 3: Top: The 20 possible environments allowed by the ice rule for a node of the triangular lattice. Bottom: the equivalent 20 vertices in the osculating path language. A path edge is drawn whenever the underlying orientation runs from North, Northwest or West to East, Southeast or South. Path steps are then concatenated into non-crossing paths.

- East boundary: horizontal edges oriented from $(n+1, j)$ to $(n, j), j=1,2, \ldots, n$ and diagonal edges oriented from $(n+1, j)$ to $(n, j+1)$ for $j=1,2, \ldots n-1$;

- North boundary: vertical edges oriented from $(i, n)$ to $(i, n+1), i=1,2, \ldots, n$ and diagonal edges oriented from $(i+1, n)$ to $(i, n+1)$ for $i=1,2, \ldots n-1$.

The boundary edge set itself is finally completed by two diagonal corner edges, and we distinguish two types of DWBC, referred to as DWBC1 and DWBC2 respectively, depending on the orientation of these corner edges:

- DWBC1: the diagonal edge oriented from $(0, n+1)$ to $(1, n)$ and the diagonal edge oriented from $(n, 1)$ to $(n+1,0)$;

or

- DWBC2: the diagonal edge oriented from $(1, n)$ to $(0, n+1)$ and the diagonal edge oriented from $(n+1,0)$ to $(n, 1)$.

The domain thus defined is clearly a portion of triangular lattice where each inner node $(i, j), i, j=1,2, \ldots, n$ is incident to six edges. A configuration of the $20 \mathrm{~V}$ model on this domain consists in the assignment of an orientation to all the inner edges satisfying the (triangular) ice rule condition that each node is incident to three outgoing and three incoming edges. Figures 1 and 2 show examples of configurations corresponding to the DWBC1 and DWBC2 ensembles respectively. The ice rule gives rise to exactly 20 possible environments around a given node, as displayed in Fig. 3, hence the name of the model. In the following, unless otherwise stated, we will be interested in enumerating the $20 \mathrm{~V}$ 
configurations without discrimination on the possible node environments. In other words, we attach the same weight 1 to each of the 20 vertices of the model.

As in the case of the $6 \mathrm{~V}$ model, the edge orientations of a configuration of the $20 \mathrm{~V}$ model may be coded bijectively by configurations of so-called osculating paths visiting the edges of the lattice and obtained as follows: we first assign to each edge of the lattice a natural orientation, namely from West to East for the horizontal edges, from North to South for the vertical edges, and from Northwest to Southeast for the diagonal edges. Each edge of the lattice is then covered by a path step if and only of its actual orientation matches the natural orientation. Note that the path steps are de facto naturally oriented from North, Northwest or West (NW for short) to East, Southeast or South (SE) and the ice rule ensures that the number of paths steps coming from NW at each node is equal to that leaving towards SE. This allows to concatenate the path steps into global paths. When four or six path steps are incident to a given node, the prescription for concatenation is the unique choice ensuring that the paths do not cross each other, even though they meet at the node at hand. Such paths are called "osculating". Note that no two paths can share a common edge. By construction, the path configuration consists of $2 n$ (respectively $2 n-1$ ) paths in the DWBC1 (respectively DWBC2) ensemble, connecting the $2 n$ edges of the West boundary plus the upper-left corner edge (respectively the $2 n-1$ edges of the West boundary) to the $2 n$ edges of the South boundary plus the lower-right corner edge (respectively the $2 n-1$ edges of the South boundary) without crossing. Figures 1 and 2 show examples of such osculating path configurations.

The simplest question we may ask about $20 \mathrm{~V}$ configuration with DWBC is that of the number $A_{n}=Z^{20 V}(n)$ of configurations for a given $n$. First we note that this number is the same for the prescriptions DWBC1 and DWBC2 due to a simple duality between the two models: performing a rotation by $180^{\circ}$ in the plane sends a configuration of arrows obeying the DWBC1 prescription to one obeying the DWBC2 and conversely (the symmetry being an involution). Indeed, the ice rule is invariant under this rotation and the boundary conditions are unchanged, except for the orientation of the corner edges which are reversed. This gives a bijection between the configurations in the two ensembles which thus have the same cardinality. In the osculating path language, the DWBC2 path configuration is obtained by taking the complement of the DWBC1 path configuration (i.e. covering uncovered edges and conversely) and rotating it by $180^{\circ}$. The configuration in Fig. 2 is the image of that of Fig. 1 by this bijection. The distinction between the two ensembles will still be significant when we address a more refined question in the next sections. The sequence of the first values of the numbers $A_{n}=Z^{20 V}(n)$ for $n$ up to 8 are listed in (1.1). The 23 configurations for $n=3$ are represented for illustration in Fig. 4

\subsection{General properties}

Following Baxter [Bax89], we may transform the 20V model into an ice model on the Kagome lattice as follows: starting from our portion of triangular lattice and splitting each node into a small triangle, say by slightly sliding each horizontal line to the North, results in a portion of Kagome lattice, as shown in Fig. 5. Clearly, any orientation of the edges of the Kagome lattice satisfying the ice rule (i.e. with two incoming and two 

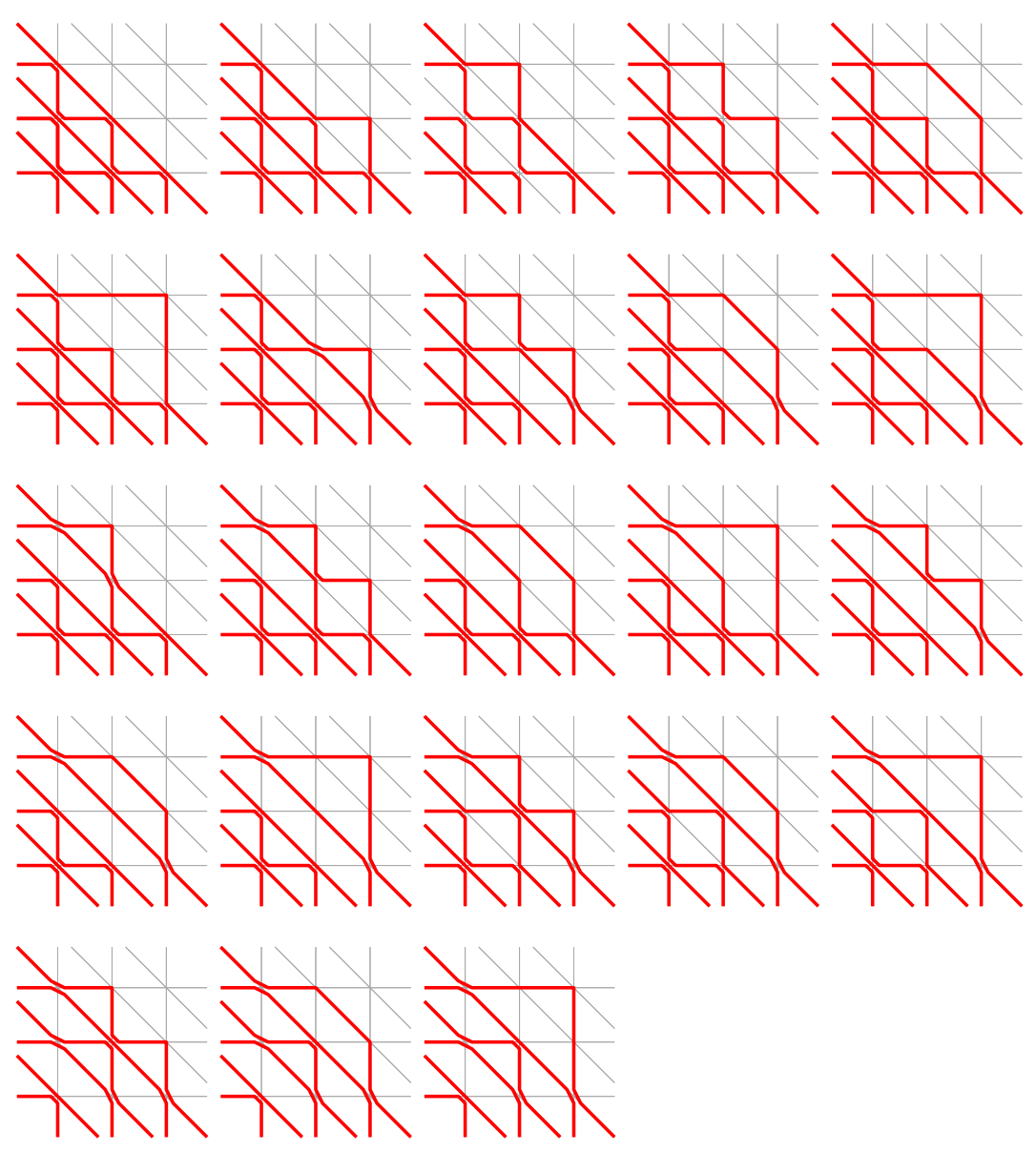

Figure 4: The 23 configurations of the $20 \mathrm{~V}$ model with DWBC1 for $n=3$, represented in the osculating path language.

outgoing edges incident to each node) results into a configuration where the six edges of the original triangular lattice satisfy the ice rule around any small triangle replacing an original node. Conversely, any orientation satisfying the ice rule on the original triangular lattice may be completed via some appropriate choice of orientation of the newly formed edges so as to create an ice model configuration on the Kagome lattice (note that the choice of orientation for the new edges is not unique in general). This construction allows to rephrase our $20 \mathrm{~V}$ model in terms of an ice model on the Kagome lattice. Let us now discuss how to recover the desired weight 1 per vertex of the $20 \mathrm{~V}$ model by some appropriate weighting of the vertex configurations around each node of the Kagome lattice. The Kagome lattice is naturally decomposed into three sublattices, denoted 1, 2 and 3 with the following choice:

- lattice 1: vertices at the crossing of a horizontal and a vertical line;

- lattice 2: vertices at the crossing of a horizontal and a diagonal line; 

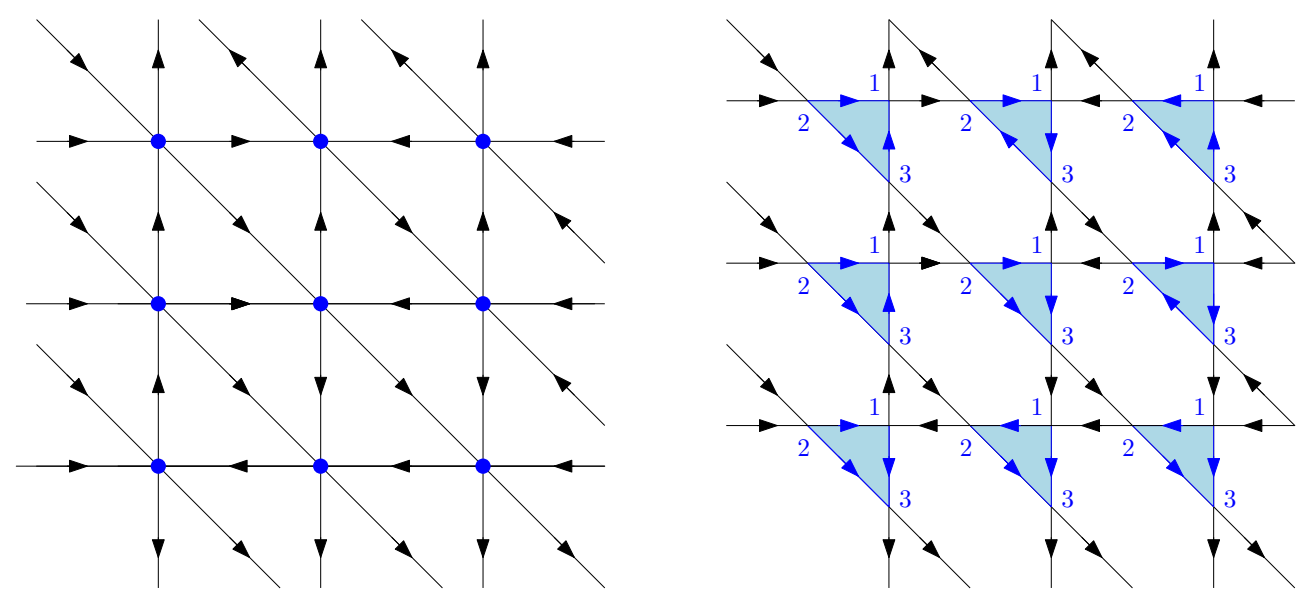

Figure 5: The deformation of the triangular lattice into the Kagome lattice by sliding the horizontal lines to the North, splitting each node into a small triangle (colored in light blue). The Kagome lattice is naturally split into three sublattices denoted 1, 2 and 3 as shown. Each configuration of the $20 \mathrm{~V}$ model (left) may be completed into a configuration satisfying the ice rule at each vertex of the Kagome lattice by some (non-unique) appropriate choice of orientation for the newly created edges (blue arrows).

- lattice 3: vertices at the crossing of a vertical and a diagonal line.

Due to the ice rule, each vertex of sublattice 1 (respectively 2 and 3) matches one of the six vertex configurations of the $6 \mathrm{~V}$ model and we may naturally weight these configurations with three weights $a_{1}, b_{1}, c_{1}$ (respectively $a_{2}, b_{2}, c_{2}$ and $a_{3}, b_{3}, c_{3}$ ) according to the rules of Fig. 6. In order to recover the desired weight 1 for each vertex of the $20 \mathrm{~V}$ model, the weights of the Kagome model must satisfy the following 10 relations $^{2}$

$$
\begin{aligned}
1 & =a_{1} a_{2} a_{3}=b_{1} a_{2} b_{3}=b_{1} a_{2} c_{3}=c_{1} a_{2} a_{3}=b_{1} c_{2} a_{3}=b_{1} b_{2} a_{3} \\
& =a_{1} b_{2} c_{3}+c_{1} c_{2} b_{3}=a_{1} b_{2} b_{3}+c_{1} c_{2} c_{3}=c_{1} b_{2} b_{3}+a_{1} c_{2} c_{3}=c_{1} b_{2} c_{3}+a_{1} c_{2} b_{3}
\end{aligned}
$$

For instance, the relation $a_{1} b_{2} c_{3}+c_{1} c_{2} b_{3}=1$ comes from the summation over the two possible orientations in the small triangle shown in Fig. 7. A possible choice of solution for the system of equations (2.1) is

$$
\left(a_{1}, b_{1}, c_{1}\right)=\frac{\alpha}{2^{1 / 3}}(1, \sqrt{2}, 1),\left(a_{2}, b_{2}, c_{2}\right)=\frac{\beta}{2^{1 / 3}}(\sqrt{2}, 1,1),\left(a_{3}, b_{3}, c_{3}\right)=\frac{\gamma}{2^{1 / 3}}(\sqrt{2}, 1,1)
$$

for any choice of $\alpha, \beta$ and $\gamma$ such that $\alpha \beta \gamma=1$. A very efficient tool in solving the $6 \mathrm{~V}$ model is the use of the so called Yang-Baxter relations which allow to deform and eventually unravel the underlying lattice into a simpler graph. In the above Kagome lattice setting, denoting by $\alpha_{1}, \cdots, \alpha_{6}$ the six orientations around a small triangle as

\footnotetext{
${ }^{2}$ Note that our definition of the weights on the Kagome lattice differ from that of Baxter in [Bax89] upon the exchange $a_{1} \leftrightarrow b_{1}$. The 20 relations for the 20 possible vertices reduce to 10 by symmetry.
} 


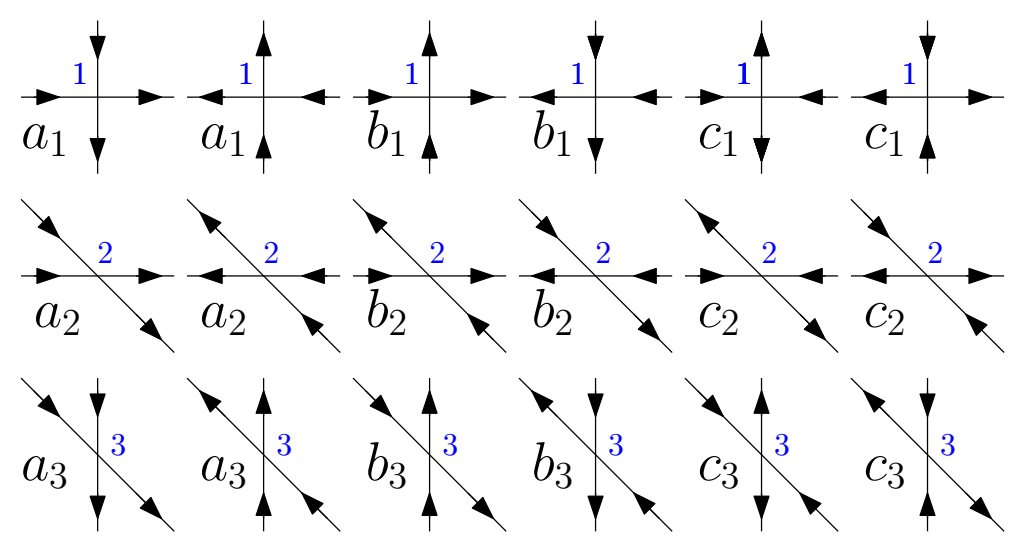

Figure 6: The weight denomination for the three copies of $6 \mathrm{~V}$ models on the Kagome lattice. The index of the weights corresponds to that of the underlying sublattice. Vertices related by a global reversing of all arrows are chosen to have the same weight.

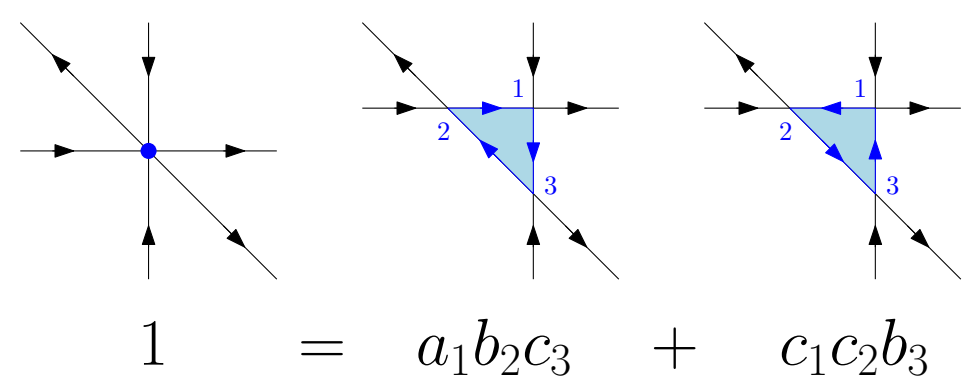

Figure 7: The relation $a_{1} b_{2} c_{3}+c_{1} c_{2} b_{3}=1$ ensuring a weight 1 for the vertex of the $20 \mathrm{~V}$ model shown on the left, as obtained by summing over the two possible orientations for the edges of the central small triangle in the equivalent Kagome lattice setting (right).
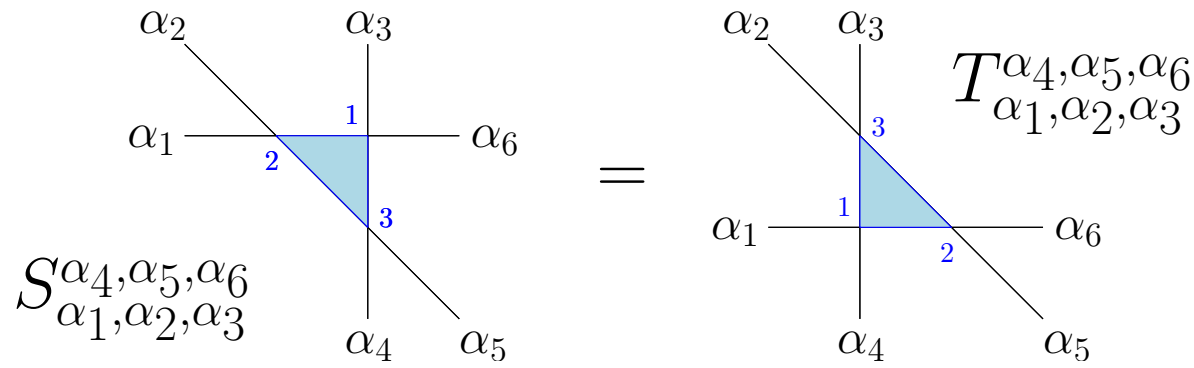

Figure 8: A schematic picture of the Yang-Baxter equation $S_{\alpha_{1}, \alpha_{2}, \alpha_{3}}^{\alpha_{4}, \alpha_{5}, \alpha_{6}}=T_{\alpha_{1}, \alpha_{2}, \alpha_{3}}^{\alpha_{4}, \alpha_{5}, \alpha_{6}}$. For fixed orientations $\alpha_{1}, \cdots, \alpha_{6}, S_{\alpha_{1}, \alpha_{2}, \alpha_{3}}^{\alpha_{4}, \alpha_{5}, \alpha_{6}}$ and $T_{\alpha_{1}, \alpha_{2}, \alpha_{3}}^{\alpha_{4}, \alpha_{5}}$ are obtained by summing over the orientations of the edges of the central triangle allowed by the ice rule on the Kagome lattice, with their associated weights of Fig. 6 . 


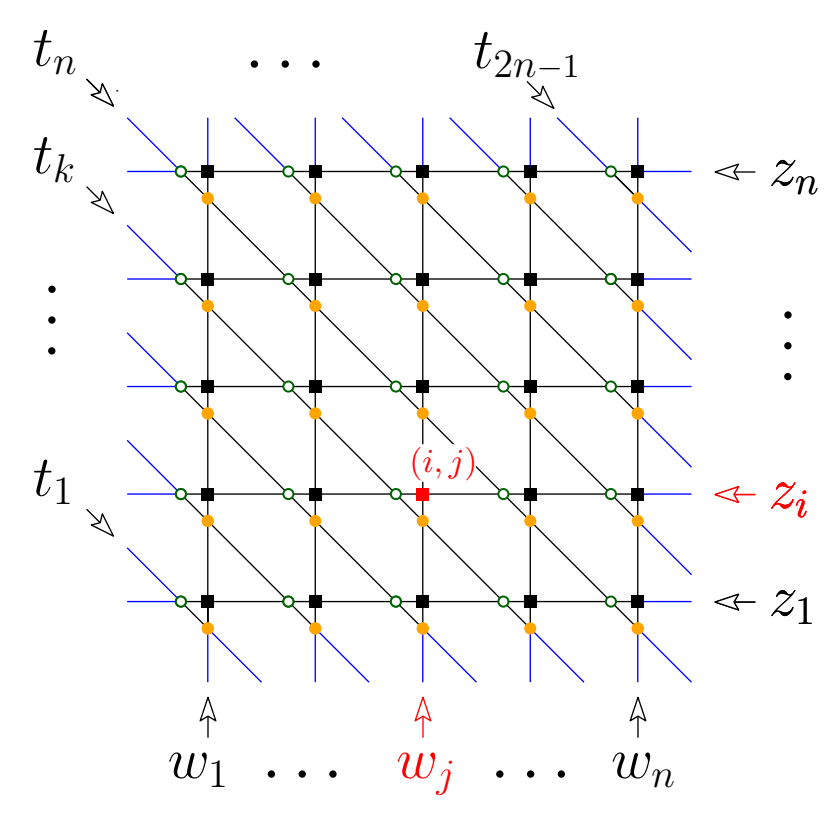

Figure 9: Spectral parameters for the Kagome lattice version of the 20V model.

shown in Fig. 8, with $\alpha_{i}=1$ if the orientation matches the natural (from NW to SE) orientation and 0 otherwise, these relations ensure that the weight $S_{\alpha_{1}, \alpha_{2}, \alpha_{3}}^{\alpha_{4}, \alpha_{6}}$ obtained by summing over the possible orientations of the edges of the small triangle is equal, for any choice of the $\alpha_{i}$ 's, to that, $T_{\alpha_{1}, \alpha_{2}, \alpha_{3}}^{\alpha_{4}, \alpha_{5}, \alpha_{6}}$, obtained by sliding the diagonal line (passing through vertices of sublattices 2 and 3 ) to the other side of the node of the sublattice 1 (see Fig. 8). In terms of the weights $\left(a_{i}, b_{i}, c_{i}\right)$, it is easily checked that this equality holds if and only if we have the three relations:

$\left(a_{1} b_{2}-b_{1} a_{2}\right) c_{3}+c_{1} c_{2} b_{3}=0, \quad\left(a_{1} b_{3}-b_{1} a_{3}\right) c_{2}+c_{1} c_{3} b_{2}=0, \quad\left(b_{2} b_{3}-a_{2} a_{3}\right) c_{1}+c_{2} c_{3} a_{1}=0$.

Note that these relations are in practice weaker than the relations $(2.1)$ in the sense that imposing (2.1) automatically implies (2.3). For instance, the relation $\left(a_{1} b_{2}-b_{1} a_{2}\right) c_{3}+$ $c_{1} c_{2} b_{3}=0$ is a direct consequence of the two identities $b_{1} a_{2} c_{3}=a_{1} b_{2} c_{3}+c_{1} c_{2} b_{3}=1$. In particular, (2.3) is satisfied by the solution (2.2), as easily verified by a direct computation.

\subsection{Integrable weight parametrization}

It is useful to introduce more general weights for our $20 \mathrm{~V}$ model, or equivalently for its Kagome reformulation, by introducing so called spectral parameters in the following way, mimicking the well known use of spectral parameters for the $6 \mathrm{~V}$ model. Let us number the horizontal lines of our lattice by $i=1,2, \ldots, n$ from bottom to top and attach a (complex) parameter $z_{i}$ to the $i$ 'th line. Similarly, we label the vertical lines by $j=1,2, \ldots, n$ from left to right and attach a parameter $w_{j}$ to the $j$ 'th line. Finally, the diagonal lines are labeled by $k=1,2 \ldots, 2 n-1$ from bottom left to top right and we attach a parameter $t_{k}$ to the $k$-th line. This labeling induces a similar labeling for the horizontal, vertical and 
diagonal lines of the Kagome lattice, see Fig. 9. Each node of the sublattice 1 is then at the crossing of a horizontal and a vertical line, hence naturally labeled by a pair $(i, j)$ with $i, j=1,2, \ldots, n$. Similarly, each node of the sublattice 2 is labeled by a pair $(i, k)$, with $i=1,2, \ldots, n$ and $k=i, i+1, \ldots, i+n-1$, while each node of the sublattice 3 corresponds to a pair $(k, j)$ with $j=1,2, \ldots, n$ and $k=j, j+1, \ldots, j+n-1$. This allows us to introduce non-homogeneous weights $\left(a_{1}(i, j), b_{1}(i, j), c_{1}(i, j)\right)$ for the configurations around vertices of the sublattice 1 according to the dictionary of Fig. 6, and similarly weights $\left(a_{2}(i, k), b_{2}(i, k), c_{2}(i, k)\right)$ and $\left(a_{3}(k, j), b_{3}(k, j), c_{3}(k, j)\right)$.

Introducing the notations

$$
A(u, v)=u-v, \quad B(u, v)=q^{-2} u-q^{2} v, \quad C(u, v)=\left(q^{2}-q^{-2}\right) \sqrt{u v},
$$

with $u, v$ and $q$ some complex numbers, we consider the following integrable weight parametrization:

$a_{1}(i, j)=A\left(z_{i}, w_{j}\right), \quad b_{1}(i, j)=B\left(z_{i}, w_{j}\right), \quad c_{1}(i, j)=C\left(z_{i}, w_{j}\right)$,

$a_{2}(i, k)=A\left(q z_{i}, q^{-1} t_{k}\right), \quad b_{2}(i, k)=B\left(q z_{i}, q^{-1} t_{k}\right), \quad c_{2}(i, k)=C\left(q z_{i}, q^{-1} t_{k}\right)$,

$a_{3}(k, j)=A\left(q t_{k}, q^{-1} w_{j}\right), \quad b_{3}(k, j)=B\left(q t_{k}, q^{-1} w_{j}\right), \quad c_{3}(k, j)=C\left(q t_{k}, q^{-1} w_{j}\right)$,

where the complex numbers $z_{i}, i-1,2, \ldots, n, w_{j}, j=1,2, \ldots, n$, and $t_{k}, k=1,2, \ldots, 2 n-1$ are arbitrarily fixed spectral parameters. The main feature of this integrable parametrization is that, for any choice of the spectral parameters, the Yang Baxter relations (2.3) are automatically satisfied for any triple $(i, j, k)$ in $(2.4)$, as easily checked by a direct computation.

The solution (2.2) of (2.1) may be recovered in this framework by choosing

$$
t_{k}=t, \quad z_{i}=q^{6} t, \quad w_{j}=q^{-6} t
$$

for all $k, i$ and $j$, leading to the homogeneous weights:

$$
\begin{array}{lll}
a_{1}=\left(q^{6}-q^{-6}\right) t, & b_{1}=\left(q^{4}-q^{-4}\right) t, & c_{1}=\left(q^{2}-q^{-2}\right) t \\
a_{2}=\left(q^{7}-q^{-1}\right) t, & b_{2}=\left(q^{5}-q\right) t, & c_{2}=\left(q^{5}-q\right) t \\
a_{3}=\left(q-q^{-7}\right) t, & b_{3}=\left(q^{-1}-q^{-5}\right) t, & c_{3}=\left(q^{-1}-q^{-5}\right) t .
\end{array}
$$

Upon taking the particular value

$$
q=\mathrm{e}^{\mathrm{i} \pi / 8},
$$

these weights reduce, using $q^{8}=-1$ and $\left(q^{4}-q^{-4}\right)=\sqrt{2}\left(q^{2}-q^{-2}\right)$, to

$$
\begin{aligned}
& \left(a_{1}, b_{1}, c_{1}\right)=\left(q^{2}-q^{-2}\right) t(1, \sqrt{2}, 1) \\
& \left(a_{2}, b_{2}, c_{2}\right)=q^{3}\left(q^{2}-q^{-2}\right) t(\sqrt{2}, 1,1) \\
& \left(a_{3}, b_{3}, c_{3}\right)=q^{-3}\left(q^{2}-q^{-2}\right) t(\sqrt{2}, 1,1)
\end{aligned}
$$

a form which matches precisely that of (2.2) with $\alpha=1, \beta=q^{3}$ and $\gamma=q^{-3}$ whenever $\left(\left(q^{2}-q^{-2}\right) t\right)^{3}=1 / 2$, namely, say

$$
t=\frac{1}{2^{1 / 3}\left(q^{2}-q^{-2}\right)}=-\frac{\mathrm{i}}{2^{5 / 6}} .
$$




\section{Mapping to a $6 \mathrm{~V}$ model with Domain Wall Boundary Condi- tions}

\subsection{Unraveling the $20 \mathrm{~V}$ configurations}

We now return to our $20 \mathrm{~V}$ model with, say the DWBC1 prescription and with a weight 1 per vertex and consider its Kagome equivalent formulation with the weights given by (2.2). As already mentioned, as solution of the equation (2.1), these weights automatically satisfy the conditions (2.3) ensuring the Yang Baxter property. This allows to deform the lattice by expelling the diagonal lines from the $n \times n$ square grid as shown in Fig. 10 . The diagonal lines with index $k \leqslant n$ are expelled towards the lower-left of the square grid and those with index $k>n$ towards the upper-right. The choice for the main diagonal $(k=n)$ is adapted to the DWBC1 prescription. For the DWBC2 prescription, the proper choice would be to move this diagonal towards the upper-right instead. In the deformed configuration, all the vertices of the sublattices 2 and 3 have been expelled outside of the $n \times n$ square grid which contains only vertices of the sublattice 1 . More interestingly, due to the ice rule and to the prescribed boundary conditions, the orientations of all the edges outside the central $n \times n$ square grid are entirely fixed (see Fig. 10), and all correspond to configurations of "type $a$ ", namely receive the weight $a_{2}$ if they belong to sublattice 2 and $a_{3}$ is they belong to the sublattice 3 . This leads to a global contribution $\left(a_{2} a_{3}\right)^{n^{2}}$ while the remaining configuration is that of a standard $6 \mathrm{~V}$ model on the $n \times n$ square grid with the celebrated Domain Wall Boundary Conditions. We immediately deduce the following:

Theorem 1. The number $A_{n}$ of configurations of the $20 \mathrm{~V}$ model with $D W B C 1,2$ on an $n \times n$ grid reads

$$
A_{n}=Z^{20 V}(n)=Z_{[1, \sqrt{2}, 1]}^{6 V}(n) .
$$

where $Z_{[a, b, c]}^{6 \mathrm{~V}}(n)$ denotes the partition function of the $6 \mathrm{~V}$ model on an $n \times n$ square grid with $D W B C$ and weights $(a, b, c)$ according to the dictionary of Fig. 11.

Proof. We indeed have

$$
\begin{aligned}
A_{n}=Z^{20 V}(n) & =\left(a_{2} a_{3}\right)^{n^{2}} Z_{\left[a_{1}, b_{1}, c_{1}\right]}^{6 V}(n)=\left(\beta \gamma\left(\frac{\sqrt{2}}{2^{1 / 3}}\right)^{2}\right)^{n^{2}} Z_{\left[\alpha \frac{1}{2^{1 / 3}}, \alpha \frac{\sqrt{2}}{2^{1 / 3}}, \alpha \frac{1}{2^{1 / 3}}\right]}^{6 V}(n) \\
& =Z_{\left[\alpha \beta \gamma\left(\frac{\sqrt{2}}{2^{1 / 3}}\right)^{2} \frac{1}{2^{1 / 3}}, \alpha \beta \gamma\left(\frac{\sqrt{2}}{2^{1 / 3}}\right)^{2} \frac{\sqrt{2}}{2^{1 / 3}}, \alpha \beta \gamma\left(\frac{\sqrt{2}}{2^{1 / 3}}\right)^{2} \frac{1}{2^{1 / 3}}\right]}^{(n)}
\end{aligned}
$$

where we used the multiplicative nature of the weights to redistribute the prefactor within the weights of the $n^{2}$ nodes of the sublattice 1 . Since $\alpha \beta \gamma=1$, the theorem follows.

Using Theorem 1 and straightforward generalizations, we will recourse to known results on the $6 \mathrm{~V}$ model with DWBC to address a number of enumeration results for the $20 \mathrm{~V}$ model. In the following, we will mainly use the osculating path formulation of the $20 \mathrm{~V}$ 


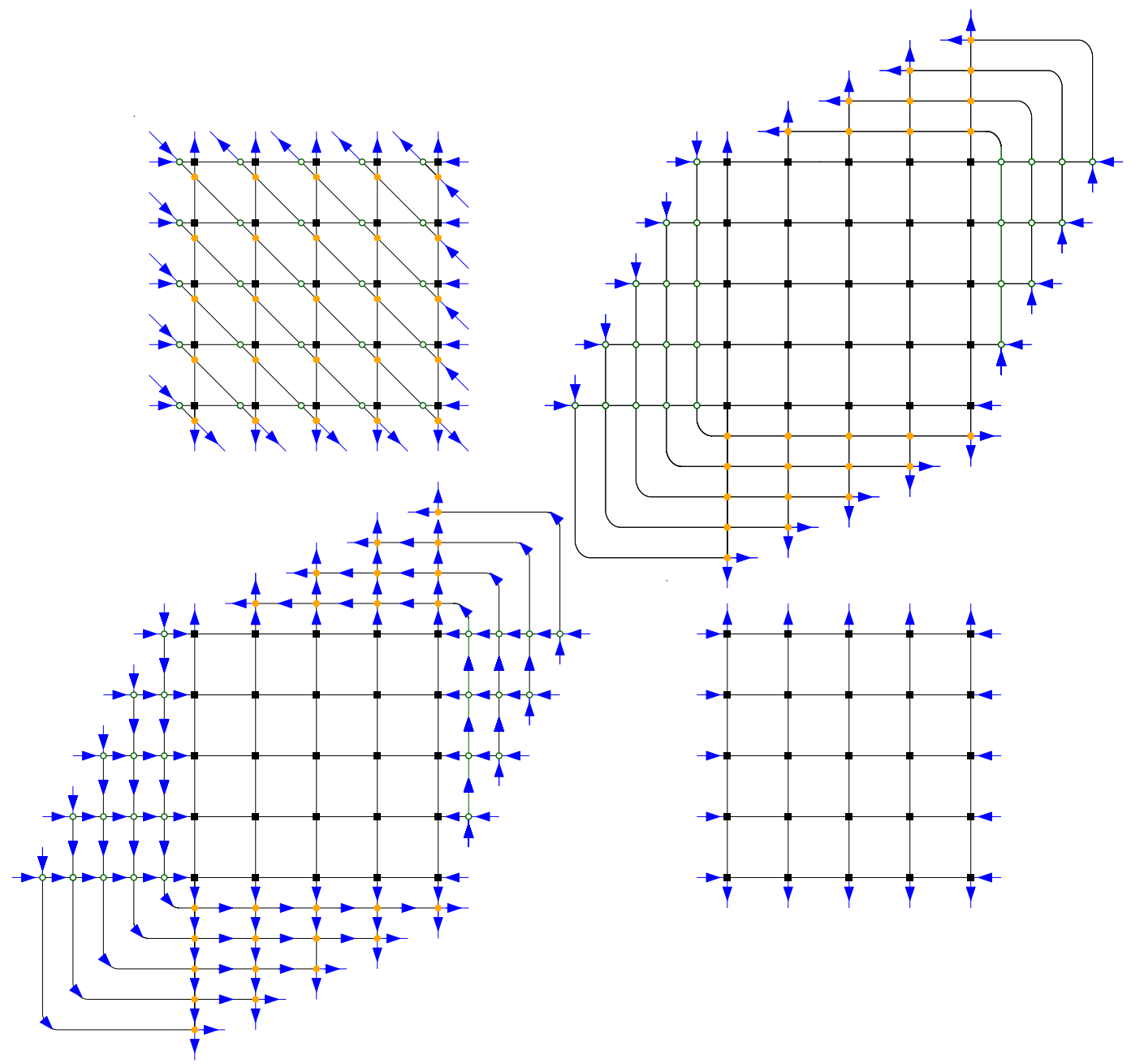

Figure 10: The unraveling of a configuration of the 20V model with DWBC1 (top left). Using the Yang Baxter property allows to deform the diagonal lines and expel them out of the central square grid (top right). Note that the main diagonal is expelled towards the lower-left of the square grid, a choice adapted to the DWBC1 prescription. Due to the ice rule and the boundary conditions, the orientations of all the edges outside of the central square grid are entirely fixed (lower left), leaving as only degrees of freedom the orientation of the edges inside the central square grid, reproducing a $6 \mathrm{~V}$ model with DWBC (lower right). 


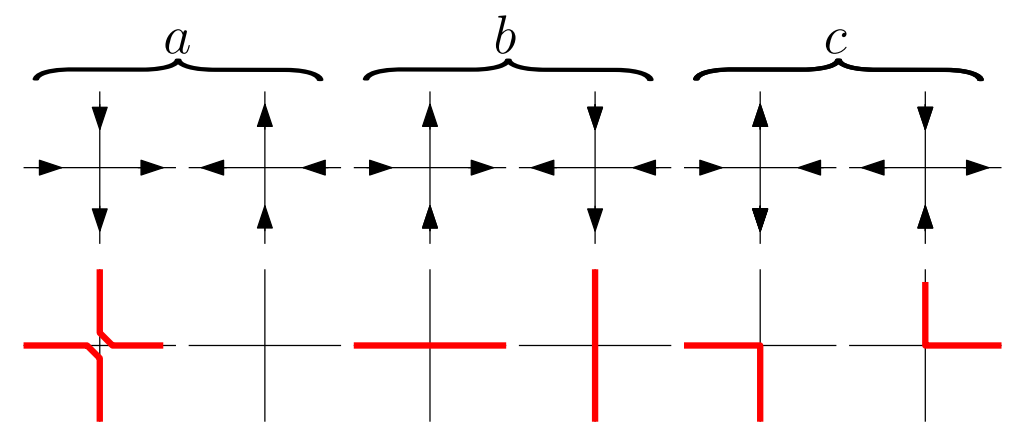

Figure 11: Vertex configurations of the $6 \mathrm{~V}$ model on the square grid and the associated weights $a, b$ and $c$ (top row) and their equivalent osculating path representation (bottom row).

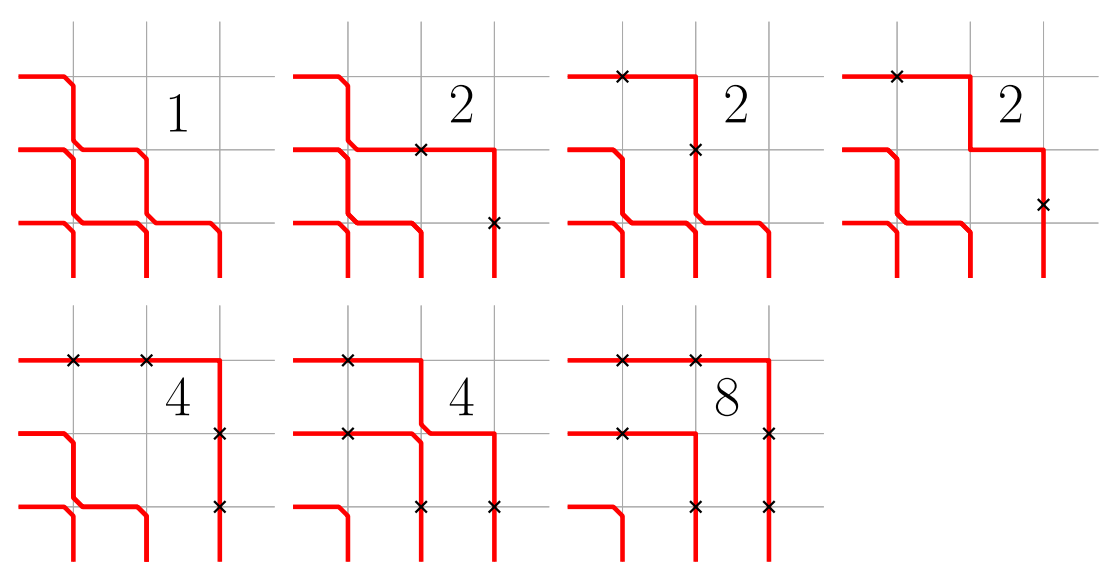

Figure 12: Illustration of the property $Z_{[1, \sqrt{2}, 1]}^{6 \mathrm{~V}}(3)=1+2+2+2+4+4+8=23$ obtained by listing all the $6 \mathrm{~V}$ model configurations with DWBC (here in the osculating path language) and their associated weight (as indicated for each configuration), corresponding to attaching a factor $\sqrt{2}$ to each node traversed vertically or horizontally by a path (as shown by cross marks). 
model and the corresponding one for the $6 \mathrm{~V}$ model according to the correspondence of Fig. 11. Fig. 12 shows how to recover the value $A_{3}=Z^{20 V}(3)=23$ from that of $Z_{[1, \sqrt{2}, 1]}^{6 V}(3)$ in the osculating path framework.

\subsection{Refined enumeration}

A refined enumeration of the $20 \mathrm{~V}$ model configurations consists, in the osculating path language, in keeping track of the position $i=\ell(\ell=1,2, \ldots, n)$ where the uppermost path $^{3}$ first hits the vertical line $j=n$. Alternatively, $\ell-1$ is the number of occupied inner vertical edges in the last column. We denote by $Z_{\ell}^{20 V_{B C 1}}=Z_{\ell}^{20 V_{B C 1}}(n)$ the number of configurations with a given $\ell$ for the DWBC1 prescription and $Z_{\ell}^{20 V_{B C 2}}$ this number for the DWBC2 prescription. These numbers are encoded in the generating functions

$$
\begin{aligned}
& \hat{Z}^{20 V_{B C 1}}(\tau)=\sum_{\ell=1}^{n} Z_{\ell}^{20 V_{B C 1}} \tau^{\ell-1} \\
& \hat{Z}^{20 V_{B C 2}}(\tau)=\sum_{\ell=1}^{n} Z_{\ell}^{20 V_{B C 2}} \tau^{\ell-1}
\end{aligned}
$$

(with an implicit $n$-dependence) which clearly satisfy $\hat{Z}^{20 V_{B C 1}}(1)=\hat{Z}^{20 V_{B C 2}}(1)=Z^{20 V}(n)$. Similarly we denote by $Z_{[1, \sqrt{2}, 1] ; \ell}^{6 V}=Z_{[1, \sqrt{2}, 1] ; \ell}^{6 V}(n)$ the number of configurations of the $6 \mathrm{~V}$ model with DWBC for which the uppermost osculating path first hits the vertical line $j=n$ at position $i=\ell$ and set

$$
\hat{Z}_{[1, \sqrt{2}, 1]}^{6 V}(\sigma)=\sum_{\ell=1}^{n} Z_{[1, \sqrt{2}, 1] ; \ell^{6 V}}^{\ell-1}
$$

with $\hat{Z}_{[1, \sqrt{2}, 1]}^{6 V}(1)=Z_{[1, \sqrt{2}, 1]}^{6 V}(n)$.

Let us now show the following:

Theorem 2. The generating polynomials $\hat{Z}^{20 V_{B C 1,2}}(\tau)$ for the refined $20 \mathrm{~V}$ model are determined by the relations

$$
\hat{Z}^{20 V_{B C 2}}(\tau)=\hat{Z}_{[1, \sqrt{2}, 1]}^{6 V}\left(\frac{1+\tau}{2}\right)=\hat{Z}^{20 V_{B C 1}}(0)+\frac{1+\tau}{2 \tau}\left(\hat{Z}^{20 V_{B C 1}}(\tau)-\hat{Z}^{20 V_{B C 1}}(0)\right) .
$$

Equivalently, coefficient-wise:

$$
\begin{aligned}
Z_{1}^{20 V_{B C 1}} & =Z_{[1, \sqrt{2}, 1] ; 1}^{6 V} \quad \text { and } \quad Z_{\ell}^{20 V_{B C 1}}=\sum_{m=\ell}^{n}\left(\begin{array}{c}
m-2 \\
\ell-2
\end{array}\right) \frac{1}{2^{m-2}} Z_{[1, \sqrt{2}, 1] ; m}^{6 V} \quad \text { for } \ell \geqslant 2, \\
Z_{\ell}^{20 V_{B C 2}} & =\sum_{m=\ell}^{n}\left(\begin{array}{c}
m-1 \\
\ell-1
\end{array}\right) \frac{1}{2^{m-1}} Z_{[1, \sqrt{2}, 1] ; m}^{6 V} .
\end{aligned}
$$

\footnotetext{
${ }^{3}$ The uppermost path corresponds to the $2 n$-th path from the bottom for DWBC1 and to the $(2 n-1)$-th path for DWBC2.
} 

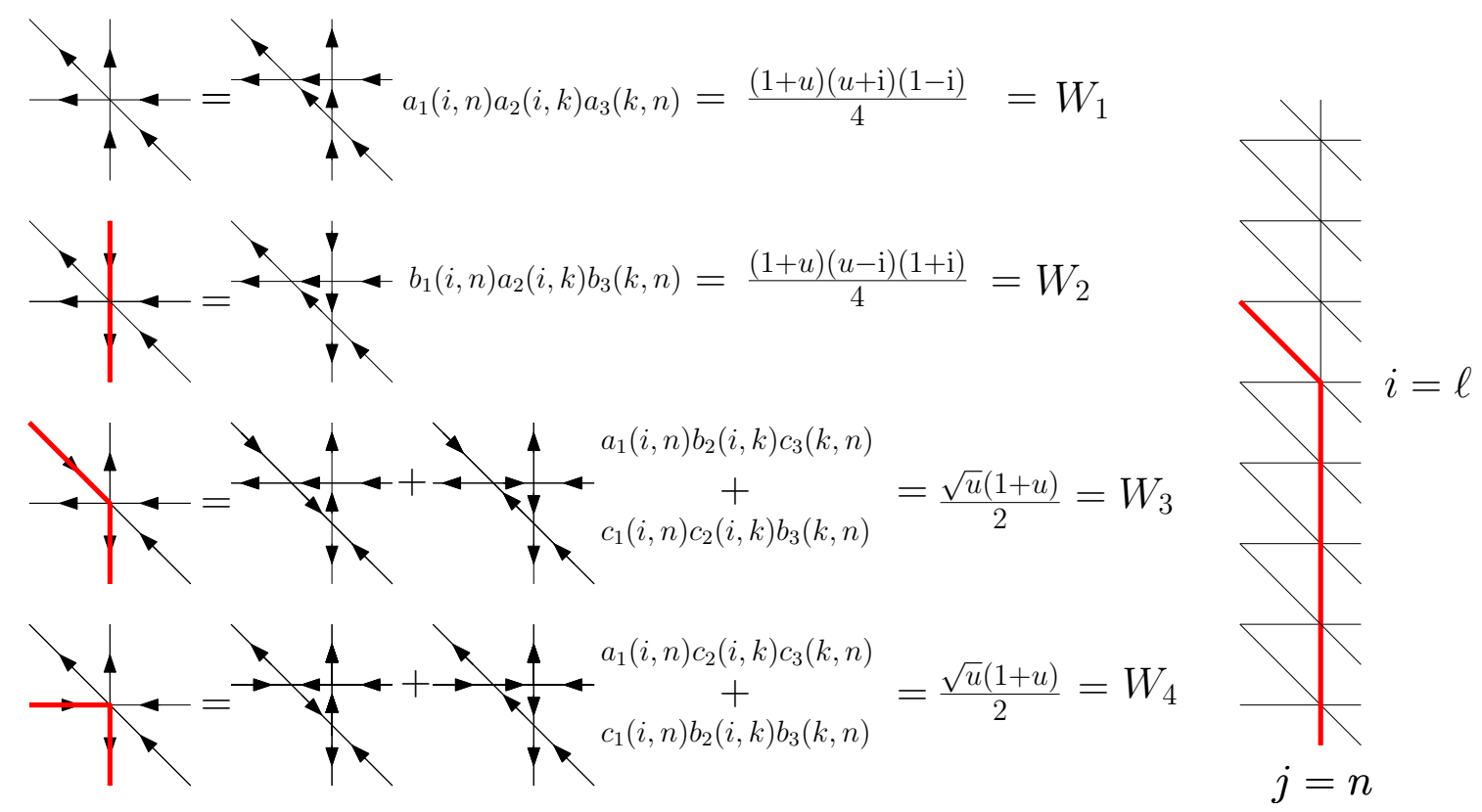

Figure 13: Left: modification of the weights of the 20V model with DWBC2 when performing the change $w_{n} \rightarrow w_{n} u$ for the spectral parameter attached to the last column from the special values (2.5), (2.6) and (2.8). We display only the four vertices which may appear in the last column due to the boundary condition at the East boundary. The weights are easily computed in the Kagome formulation, with the result $W_{1}, \ldots, W_{4}$ shown, satisfying $W_{i} \rightarrow 1$ when $u \rightarrow 1$, as required. Right: the configuration in the last column is, from bottom to top, made of a sequence of vertices weighted by $W_{2}$, then of a single vertex with weight $W_{3}$ or $W_{4}$ (hitting point) and finally of a complementary sequence of vertices weighted by $W_{1}$.

Note that the second relation in (3.2) may alternatively be rewritten as

\section{Corollary 3.}

$$
\hat{Z}^{20 V_{B C 1}}(\tau)=\frac{2 \tau}{1+\tau} \hat{Z}_{[1, \sqrt{2}, 1]}^{6 V}\left(\frac{1+\tau}{2}\right)+\frac{1-\tau}{1+\tau} \hat{Z}_{[1, \sqrt{2}, 1]}^{6 V}(0)
$$

To prove Theorem 2, let us start with the simplest case of DWBC2. The generating function $\hat{Z}^{20 V_{B C 2}}(\tau)$ may easily be obtained, in the equivalent Kagome formulation of the $20 \mathrm{~V}$ model, by slightly modifying the spectral parameter $w_{n}$ for the last column. Choosing the integrable parametrization (2.4) for the Kagome vertex weights with $q$ as in (2.6), $t_{k}=t$ as in (2.8) for all $k, z_{i}=q^{6} t$ for all $i$ and $w_{j}=q^{-6} t$ for all $j<n$ while $w_{n}=q^{-6} t u$ for some parameter $u$, only the weights $\left(a_{1}(i, n), b_{1}(i, n), c_{1}(i, n)\right)$ and $\left(a_{3}(k, n), b_{3}(k, n), c_{3}(k, n)\right)$ are modified with respect to the homogeneous values of $(2.7)$. The new values are

$$
\begin{array}{lll}
a_{1}(i, n)=\left(q^{2} u-q^{-2}\right) t, & b_{1}(i, n)=\left(q^{4}-q^{-4} u\right) t, & c_{1}(i, n)=\left(q^{2}-q^{-2}\right) \sqrt{u} t \\
a_{3}(i, n)=q^{-3}\left(q^{4}-q^{-4} u\right) t, & b_{3}(i, n)=q^{-3}\left(q^{2}-q^{-2} u\right) t, & c_{3}(i, n)=q^{-3}\left(q^{2}-q^{-2}\right) \sqrt{u} t
\end{array}
$$




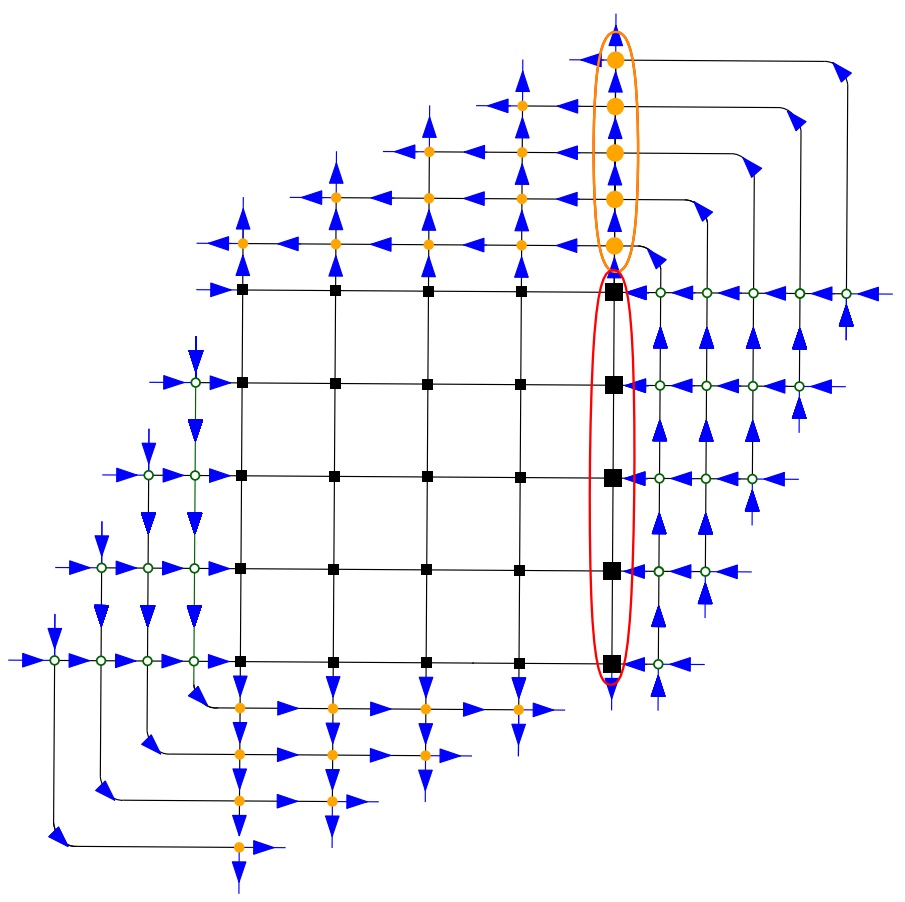

Figure 14: In the unraveled configuration (here for DWBC2, hence with the main diagonal expelled towards the upper-right corner), changing $w_{n} \rightarrow w_{n} u$ modifies only those weights corresponding to the encircled sets of nodes. The top set results into a global factor while the bottom set corresponds to a change of the $6 \mathrm{~V}$ weights in the last column (see text).

This in turns leaves all the vertex weights of the $20 \mathrm{~V}$ model equal to 1 , except for those of the last column $(j=n)$. Due to the boundary condition on the right of this column, only four vertex configurations are possible, as displayed in Fig. 13, corresponding to a vertex not visited by the uppermost path (weight $W_{1}$ ), a vertex crossed vertically by the uppermost path (weight $W_{2}$ ), or a vertex where the uppermost path hits the last column for the first time after a diagonal step (weight $W_{3}$ ) or a horizontal step (weight $W_{4}$ ). The respective new weights $W_{1}, \ldots W_{4}$ are easily computed from the new Kagome weights above (see Fig. 13), with the result:

$$
W_{1}=\frac{(1+u)(u+\mathrm{i})(1-\mathrm{i})}{4}, \quad W_{2}=\frac{(1+u)(u-\mathrm{i})(1+\mathrm{i})}{4} \quad W_{3}=W_{4}=\frac{\sqrt{u}(1+u)}{2} .
$$

Clearly, a configuration for which the uppermost path hits the last column at position $\ell$ corresponds to the last column formed (from bottom to top) of $\ell-1$ vertices with weight $W_{2}$ (below the hitting point), one vertex with weight $W_{3}$ or $W_{4}$ (the hitting point) and $(n-\ell)$ vertices with weight $W_{1}$ (above the hitting point). Note the crucial property $W_{3}=W_{4}$ which ensures that configurations, when hitting the last column, are weighted independently on the way (horizontal or diagonal) they reach this column and receive the weight $W_{2}^{\ell-1} W_{3} W_{1}^{n-\ell}$. To summarize, setting $w_{n}=q^{-6} t u$ instead of $q^{-6} t$ changes the 
partition function $Z^{20 V}(n)$ into the quantity

$$
\sum_{\ell=1}^{n} Z_{\ell}^{20 V_{B C 2}}\left(\frac{(1+u)(u-\mathrm{i})(1+\mathrm{i})}{4}\right)^{\ell-1} \frac{\sqrt{u}(1+u)}{2}\left(\frac{(1+u)(u+\mathrm{i})(1-\mathrm{i})}{4}\right)^{n-\ell} \text {. }
$$

This quantity may be computed alternatively in the $6 \mathrm{~V}$ model language by unraveling our configuration of the $20 \mathrm{~V}$ model, or more precisely its Kagome lattice equivalent, as we did in the previous section. Indeed, the Yang Baxter relations still hold with the modified value of $w_{n}$. Note that since we are now considering the DWBC2 prescription, the diagonal line $k=n$ must be moved towards the upper-right of the central square grid. As shown in Fig. 14, changing $w_{n}$ from $q^{-6} t$ to $q^{-6} t u$ generates, compared with the fully homogeneous case, the following modifications:

- a global factor $\left(\frac{A\left(q t, q^{-7} t u\right)}{A\left(q t, q^{-7} t\right)}\right)^{n}=\left(\frac{q^{4}-q^{-4} u}{q^{4}-q^{-4}}\right)^{n}=\left(\frac{1+u}{2}\right)^{n}$ for the vertices of the sublattice 3 crossing the vertical line $j=n$ (top encircled set of nodes in Fig. 14);

- a change of the weights $(1, \sqrt{2}, 1)$ for the equivalent DWBC $6 \mathrm{~V}$ model in the last column of the central square grid into weights:

$\left(\frac{A\left(q^{6} t, q^{-6} t u\right)}{A\left(q^{6} t, q^{-6} t\right)}, \frac{B\left(q^{6} t, q^{-6} t u\right)}{B\left(q^{6} t, q^{-6} t\right)}, \frac{C\left(q^{6} t, q^{-6} t u\right)}{C\left(q^{6} t, q^{-6} t\right)}\right)=\left(1 \times \frac{(u+\mathrm{i})(1-\mathrm{i})}{2}, \sqrt{2} \times \frac{1+u}{2}, 1 \times \sqrt{u}\right)$

(bottom encircled set of nodes in Fig. 14).

Gathering the weights in the last column for a configuration where the uppermost path hits the last column at position $i=\ell$ (with the same argument as for the $20 \mathrm{~V}$ model), we obtain for the modified partition function (3.4) the alternative expression

$$
\left(\frac{1+u}{2}\right)^{n} \sum_{\ell=1}^{n} Z_{[1, \sqrt{2}, 1] ; \ell}^{6 V}\left(\frac{1+u}{2}\right)^{\ell-1} \sqrt{u}\left(\frac{(u+\mathrm{i})(1-\mathrm{i})}{2}\right)^{n-\ell} .
$$

Equating (3.4) and (3.5) leads directly to the announced relation (3.2) identifying $\hat{Z}^{20 V_{B C 2}}(\tau)$ to $\hat{Z}_{[1, \sqrt{2}, 1]}^{6 V}\left(\frac{1+\tau}{2}\right)$ upon setting $\tau=\mathrm{i} \frac{u-\mathrm{i}}{u+\mathrm{i}}$.

We may now easily repeat the argument in the case of the DWBC1 prescription. The modified weights are the same as those listed in Fig. 13 but the lower right vertex $(i=1, j=n)$ involves new modified vertex weights $W_{5}, W_{6}$ and $W_{7}$ listed in Fig. 15. Again we note the crucial property $W_{5}=W_{6}$ which ensures that configurations are weighted independently on the way the penultimate (just below the uppermost) path reaches the $(i=1, j=n)$ vertex. For $\ell>1$, a configuration where the uppermost path hits the last column at position $i=\ell$ receives a weight $W_{5} W_{2}^{\ell-2} W_{3} W_{1}^{n-\ell}$ while for $\ell=1$, it receives the weight $W_{7} W_{1}^{n-1}$. The partition function $Z^{20 V}$ is now transformed into the quantity

$$
\begin{aligned}
& Z_{1}^{20 V_{B C 1}}\left(\frac{\sqrt{u}(1+u)}{2}\right)\left(\frac{(1+u)(u+\mathrm{i})(1-\mathrm{i})}{4}\right)^{n-1} \\
& +\sum_{\ell=2}^{n} Z_{\ell}^{20 V_{B C 1}}\left(\frac{1+u}{2}\right)^{2}\left(\frac{(1+u)(u-\mathrm{i})(1+\mathrm{i})}{4}\right)^{\ell-2} \frac{\sqrt{u}(1+u)}{2}\left(\frac{(1+u)(u+\mathrm{i})(1-\mathrm{i})}{4}\right)^{n-\ell} .
\end{aligned}
$$




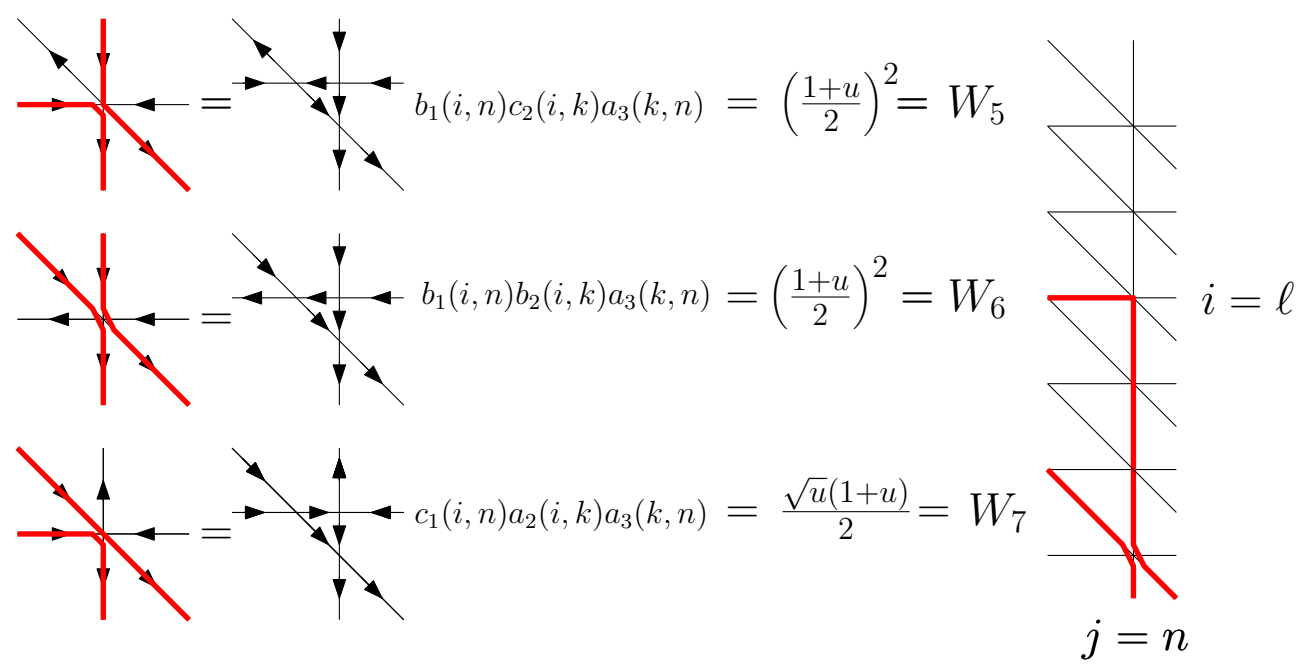

Figure 15: Left: modification of the weights of the 20V model with DWBC1 when performing the change $w_{n} \rightarrow w_{n} u$ for the spectral parameter attached to the last column from the special values (2.5), (2.6) and (2.8). We display only the three new vertices specific to DWBC1 (and thus not encountered in Fig. 13), corresponding to the three possible environments of the lowerright node. The associated values $W_{5}, W_{6}, W_{7}$ satisfy $W_{i} \rightarrow 1$ when $u \rightarrow 1$, as required. Right: a sample configuration of the last column.

As before, this quantity must be equal to that of (3.5) for the equivalent $6 \mathrm{~V}$ model after unraveling (note that the diagonal line with $k=n$ must now be moved to the lower-left of the central square grid but this does not alter the global prefactor in (3.5)). Setting $\tau=\mathrm{i} \frac{u-\mathrm{i}}{u+\mathrm{i}}$ and equating the two expressions (3.6) and (3.5) leads directly to the announced relation (3.2) between $\hat{Z}^{20 V_{B C 1}}(\tau)$ and $\hat{Z}_{[1, \sqrt{2}, 1]}^{6 V}\left(\frac{1+\tau}{2}\right)$. This completes the proof of Theorem 2 and its Corollary 3.

\subsection{Free energy and partition function from the $6 \mathrm{~V}$ solution}

From the above identifications, we may now rely on known results on the $6 \mathrm{~V}$ model with DWBC to explore the statistic of the $20 \mathrm{~V}$ model with DWBC1 or DWBC2. A first result concerns the asymptotics of $A_{n}=Z^{20 V}(n)$ for large $n$, directly given from that of $Z_{[1, \sqrt{2}, 1]}^{6 V}(n)$. For $(a, b, c)=(1, \sqrt{2}, 1)$, the value of the anisotropy parameter is $\Delta=\frac{a^{2}+b^{2}-c^{2}}{2 a b}=\frac{1}{\sqrt{2}}$, meaning that the $6 \mathrm{~V}$ model is in the so-called "disordered phase" region. Using the standard parametrization

$$
a=\rho \sin (\lambda-\phi), \quad b=\rho \sin (\lambda+\phi), \quad c=\rho \sin (2 \lambda),
$$


with $|\phi|<\lambda$, where we may take in our case $\lambda=3 \pi / 8, \phi=\pi / 8$ and $\rho=\sqrt{2}$, the exponential growth of $Z_{[1, \sqrt{2}, 1]}^{6 V}(n)$, hence of $Z^{20 V}(n)$ at large $n$ is known to be [BF06, ZJ00].

$$
A_{n}=Z^{20 V}(n)=Z_{[1, \sqrt{2}, 1]}^{6 V}(n) \underset{n \rightarrow \infty}{\sim}\left(\rho \frac{\pi(\cos (2 \phi)-\cos (2 \lambda))}{4 \lambda \cos \left(\frac{\pi t}{2 \lambda}\right)}\right)^{n^{2}}=\left(\frac{4}{3}\right)^{\frac{3}{2} n^{2}}
$$

hence a free energy per site

$$
f=\frac{3}{2} \log \frac{4}{3} .
$$

If we use for the $6 \mathrm{~V}$ model a parametrization of the form (2.4) by taking

$$
a(i, j)=z_{i}-w_{j}, \quad b(i, j)=q^{-2} z_{i}-q^{2} w_{j}, \quad c(i, j)=\left(q^{2}-q^{-2}\right) \sqrt{z_{i} w_{j}},
$$

for the weights at the nodes $(i, j)$, the homogeneous values $(a, b, c)=(1, \sqrt{2}, 1)$ correspond to choosing ${ }^{4}$

$$
q=\mathrm{e}^{\mathrm{i} \pi / 8} \quad z_{i}=z=\frac{1}{1-q^{4}}=\frac{1+\mathrm{i}}{2} \quad w_{j}=w=\frac{q^{4}}{1-q^{4}}=\frac{\mathrm{i}-1}{2}
$$

for all $i, j=1, \ldots, n$.

For arbitrary spectral parameters, the partition function of the $6 \mathrm{~V}$ model with DWBC is obtained via the celebrated so-called Izergin-Korepin determinant formula [Kor82, Ize87, ICK92]:

$$
Z^{6 V}=\frac{\prod_{i=1}^{n} c(i, i) \prod_{i, j=1}^{n}(a(i, j) b(i, j))}{\prod_{1 \leqslant i<j \leqslant n}\left(z_{i}-z_{j}\right)\left(w_{j}-w_{i}\right)} \operatorname{det}_{1 \leqslant i, j \leqslant n}\left(\frac{1}{a(i, j) b(i, j)}\right)
$$

with $a(i, j), b(i, j)$ and $c(i, j)$ as in (3.7). This expression is singular when the $z_{i}$ and $w_{j}$ tend to their homogeneous values (3.8) but we will explain in Section 5 how to circumvent this problem.

\section{Quarter-turn symmetric Domino tilings of a holey Aztec square}

Leaving the ice models aside for a while, we now turn to a different class of problems, that of domino tilings of conic domains. Our interest in these problems is motivated by the observation that their configurations are enumerated by the same sequence $A_{n}$ of (1.1). A proof of this remarkable fact will be given in Section 5 below. 


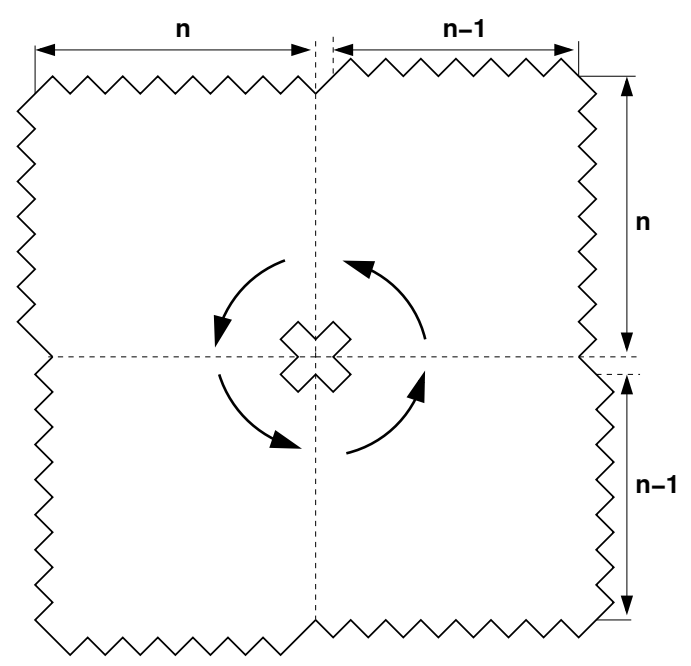

(a)

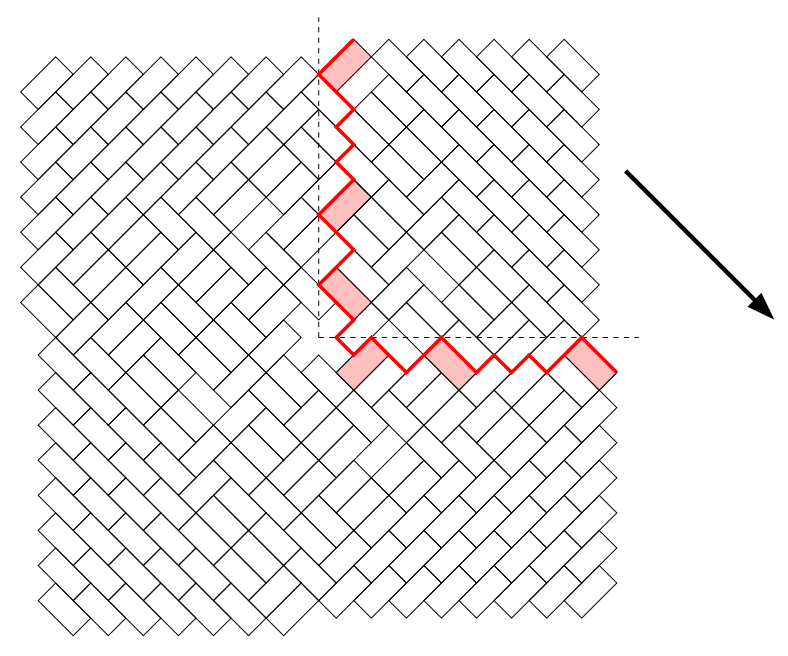

(b)

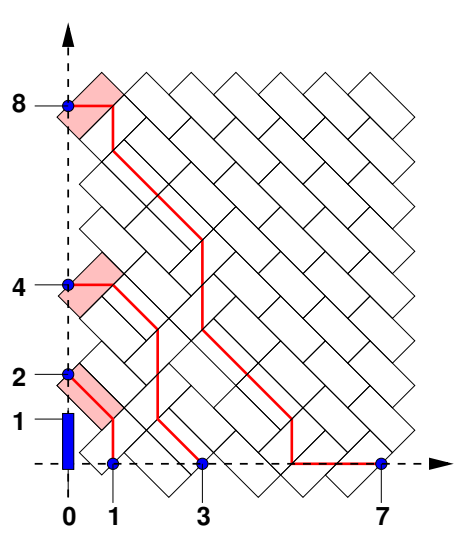

(c)

Figure 16: (a) The quasi-square Aztec-like domain $\mathcal{A}_{n}$ with a central cross-shaped hole. (b) A sample tiling configuration of $\mathcal{A}_{n}$ invariant under quarter-turn rotations around the central cross. The dashed lines identify a fundamental domain w.r.t. rotational symmetry. We have shaded the dominos from the top right domain that touch the vertical dashed line: these determine the zig-zag boundary of the fundamental domain. Summing over all positions of shaded dominos and all tiling configurations of the corresponding fundamental domain yields the desired number of tiling configurations of $\mathcal{A}_{n}$ that are quarter-turn symmetric. (c) The tiling of the fundamental domain is in bijection with configurations of non-intersecting Schröder paths with fixed ends on the shaded dominos, with symmetric positions (here $1,3,7$ and $2,4,8$ ) on the horizontal and vertical axes. 


\subsection{Definition of the model: domain and domino tilings}

We consider the domain $\mathcal{A}_{n}$ depicted in Fig. 16 (a) forming a quasi-square of Aztec-like shape of size $2 n \times 2 n$, with a cross-shaped hole in the middle. We wish to enumerate the domino tilings of this domain that are invariant under a quarter-turn rotation (i.e. of angle $\pi / 2$ ) around the center of the cross. Equivalently, identifying the domain modulo quarter-turns, the problem is equivalent to domino tilings of a cone with a hole at its apex.

The present setting can be viewed as an Aztec-like generalization of that derived in [Lal03, Kra06] to reformulate Andrews' DPP [And80]. There, the DPP were shown to be in bijection with the rhombus tiling configurations of a quasi-regular hexagon (of shape $(n, n+2, n, n+2, n, n+2))$ with a central triangular hole of size 2 , invariant under rotations of angle $2 \pi / 3$.

\subsection{Counting the tilings via Schröder paths}

To perform the desired enumeration, let us delineate a fundamental domain w.r.t. the rotational symmetry of the tiling configurations as shown in Fig. 16 (b). We draw axes centered at the center of the cross, and concentrate on the first quadrant. For any tiling configuration, we shade the dominos that touch the vertical half-axis by a corner and belong to the first quadrant. As shown in Fig. 16, these delineate a zig-zag boundary, with a "defect" protruding to the left for each shaded domino. We then draw a copy of this zig-zag boundary, obtained by rotation of $-\pi / 2$ : these delimit the fundamental domain of the tiling. Note that the fundamental domain is entirely determined by the set of shaded dominos. To characterize the tiling configurations of such a fundamental domain, we use the standard mapping to non intersecting Schröder paths (Fig. 16 (c)) obtained by first bi-coloring the underlying (tilted) square lattice so that say the center of the cross is black, and by applying the following dictionary:
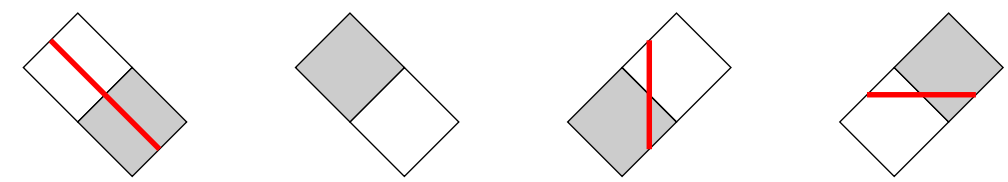

The Schröder paths are drawn on another $\mathbb{Z}^{2}$ lattice, with coordinates shifted by $1 / 2$ on both axes. When oriented from their starting point on the (new) horizontal axis to their endpoints on the (new) vertical axis, these paths have left, up and diagonal steps $(-1,0),(0,1),(-1,1)$ respectively. The endpoints of the paths belong to the shaded dominos and occupy positions $\left(0,1+i_{k}\right)$ for some integers $i_{1}, \ldots, i_{\ell} \in[1, n-1]$ since the first position $(i=0)$ is forbidden by the cross-shaped hole. The corresponding starting points occupy positions $\left(i_{k}, 0\right)$ for the same set $\left\{i_{1}, \ldots, i_{\ell}\right\}$. Moreover, from the above

\footnotetext{
${ }^{4}$ Here when computing $c$, we adopt the convention that $\sqrt{\left(q^{2}-q^{-2}\right)^{2}}=\left(q^{2}-q^{-2}\right)$. Choosing the other branch of the square root would yield $c=-1$ but, from the relation $Z_{[1, \sqrt{2}, 1]}^{6 V}(n)=(-1)^{n} Z_{[1, \sqrt{2},-1]}^{6 V}(n)$ for the $6 \mathrm{~V}$ model with DWBC, we would eventually recover for $Z_{[1, \sqrt{2}, 1]}^{6 V}(n)$ the very same expression as that (5.2) presented in Section 5 below.
} 
construction, the endpoints of the paths are on the NW border of the leftmost (white) half of the shaded dominos, and therefore the last step of all the paths can only be either left or diagonal, but cannot be up: we shall call these restricted Schröder paths. Finally, the total number of tiling configurations of $\mathcal{A}_{n}$ with quarter-turn symmetry is obtained by summing over all possible positions and orientations of the shaded dominos, i.e. over all possible positions $i_{1}, \ldots, i_{\ell}$ of the endpoints, of the number of configurations of nonintersecting restricted Schröder paths with these particular endpoints and their associated symmetric starting points.

Schröder paths are readily enumerated via the generating function

$$
S(r, s):=\sum_{i, j \geqslant 0} r^{i} s^{j} S_{i, j}=\frac{1}{1-r-s-r s}
$$

where $S_{i, j}$ is the number of Schröder paths from point $(i, 0)$ to point $(0, j)$. We may think of $r, s$ as generators of left and up steps respectively, and $r s$ as the generator of a diagonal step: in any term of the expansion of the generating function of the form $r^{\ell} s^{u}(r s)^{d}=r^{i} s^{j}$ for respectively $\ell, u, d$ left, up and diagonal steps, we indeed have $i=\ell+d$ and $j=u+d$. Restricted Schröder paths require that the last step cannot be up. If we now denote by $\tilde{S}_{i, j}$ the number of restricted Schröder paths from $(i, 0)$ to $(0, j)$, then the desired entries $\tilde{S}_{i, j+1}$ of the Gessel-Viennot matrix are generated by:

$$
\sum_{i, j \geqslant 0} \tilde{S}_{i, j+1} r^{i} s^{j}=\frac{1}{s}(r s S(r, s)+r(S(r, s)-S(r, 0)))=\frac{2 r}{(1-r)(1-r-s-r s)}
$$

where we have decomposed the paths according to their last step, respectively with weight $r s$ (if diagonal) or $r$ (if left) and preceded respectively by an arbitrary Schröder path, generated by $S(r, s)$, or by an arbitrary Schröder path with a height difference of at least one, generated by $(S(r, s)-S(r, 0))$. The global $1 / s$ is simply due to the fact that we attach a weight $s^{j}$ in our definition instead of the natural $s^{j+1}$ associated with a height difference $j+1$. Note that, for $i=0, \tilde{S}_{0, j+1}=0$ as there is no restricted Schröder path with only vertical steps, while, for $j=0, \tilde{S}_{i, 1}=2 i$.

The partition function of the tiling model is given by the following:

Theorem 4. The number $T_{4}\left(\mathcal{A}_{n}\right)$ of quarter-turn symmetric tilings of the domain $\mathcal{A}_{n}$ is given by the $n \times n$ determinant:

$$
T_{4}\left(\mathcal{A}_{n}\right)=\operatorname{det}\left(\mathbb{I}_{n}+M_{n}\right)
$$

where $\left(\mathbb{I}_{n}\right)_{i, j}=\delta_{i, j}$ and $\left(M_{n}\right)_{i, j}=\tilde{S}_{i, j+1}$ for $i, j=0,1, \ldots, n-1$.

Proof. Recall the Lindström Gessel-Viennot (LGV) determinant formula [Lin73, GV85]: the number of non-intersecting restricted Schröder paths with fixed starting points $\left(i_{k}, 0\right)$ and endpoints $\left(0,1+i_{k}\right), k=1,2, \ldots, \ell$ is given by the sub-determinant $\left|M_{n}\right|_{i_{1}, \ldots, i_{\ell}}^{i_{1}, \ldots, i_{\ell}}$ of the matrix $M_{n}$ obtained by keeping rows and columns with labels $i_{1}, \ldots, i_{\ell}$ (corresponding 
respectively to the starting and ending points). The theorem follows from the standard Cauchy-Binet identity:

$$
\operatorname{det}\left(\mathbb{I}_{n}+M_{n}\right)=\sum_{\ell=0}^{n} \sum_{0 \leqslant i_{1}<i_{2} \cdots<i_{\ell} \leqslant n-1}\left|M_{n}\right|_{i_{1}, \ldots, i_{\ell}}^{i_{1}, \ldots, i_{\ell}},
$$

which realizes the desired sum over all possible choices of symmetric starting and endpoints. Note that the sum includes paths with $i_{1}=0$, which do not contribute as $\left(M_{n}\right)_{0, j}=\tilde{S}_{0, j+1}=0$.

Using the generating function for the infinite matrix $\mathbb{I}+M$ :

$$
T_{4}(r, s)=\sum_{i, j \geqslant 0}(\mathbb{I}+M)_{i, j} r^{i} s^{j}=\frac{1}{1-r s}+\frac{2 r}{(1-r)(1-r-s-r s)}
$$

we easily generate the numbers $T_{4}\left(\mathcal{A}_{n}\right)$ from

$$
T_{4}\left(\mathcal{A}_{n}\right)=\operatorname{det}_{0 \leqslant i, i \leqslant n-1}\left(\left.\left(\frac{1}{1-r s}+\frac{2 r}{(1-r)(1-r-s-r s)}\right)\right|_{r^{i} s^{j}}\right)
$$

(here $\left.f(r, s)\right|_{r^{i} s^{j}}$ denotes the coefficient of $r^{i} s^{j}$ in a double series expansion of $f(r, s)$ in $r$ and $s)$. Remarkably, these numbers match precisely the sequence $A_{n}$ of (1.1).

\subsection{Refined enumeration}

In this section, we consider refined quarter-turn symmetric tiling configurations of $\mathcal{A}_{n}$. The origin of the refinement is best explained in the formulation as non-intersecting Schröder paths of Fig. 16 (c). Comparing these configurations to those attached to DPP in [Kra06], we are led to consider the following two statistics for restricted Schröder paths. For each such path $p$ from $(i, 0) \rightarrow(0, j)$, where $0 \leqslant i \leqslant n-1$ and $1 \leqslant j \leqslant n$, we define numbers $\ell_{1}(p)$ and $\ell_{2}(p)$ as:

- if $j<n, \ell_{1}(p)=\ell_{2}(p)=0$.

- if $j=n, \ell_{1}(p)$ is the $\mathrm{x}$ coordinate of the last point with y coordinate $\leqslant n-1$.

- if $j=n, \ell_{2}(p)$ is the $\mathrm{x}$ coordinate of the first point with y coordinate equal to $n$.

This allows to define the refined numbers $T_{4, k}^{(m)}\left(\mathcal{A}_{n}\right), m=1,2$ of non-intersecting restricted Schröder path configurations $\mathcal{P}$ with $^{5} \ell_{m}(\mathcal{P}):=\sum_{p \in \mathcal{P}} \ell_{m}(p)=k$, for some $k=0,1, \ldots, n-1$. We refer to such models as type 1 or 2 according to the value of $m$. In turn, these correspond to a refinement of the quarter-turn symmetric tiling configurations of $\mathcal{A}_{n}$ :

\footnotetext{
${ }^{5}$ Note that at most one path contributes to this sum, namely the topmost one if it hits the height $n$.
} 
Type 1: $\ell_{1}(\mathcal{P})=k$ iff the top row of the fundamental domain corresponds to the following tiling pattern (with $k$ or $k-1$ up-right dominos):
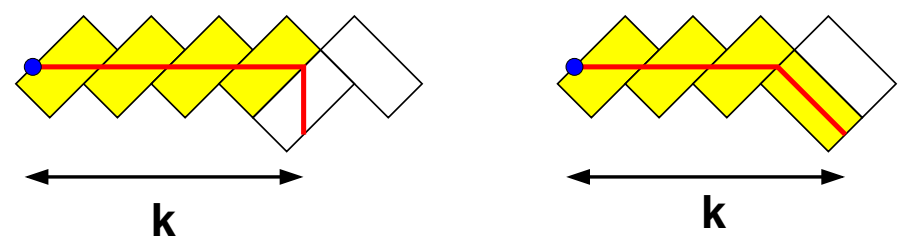

Type 2: $\ell_{2}(\mathcal{P})=k$ iff there are exactly $k$ up-right dominos in the top row of the fundamental domain.

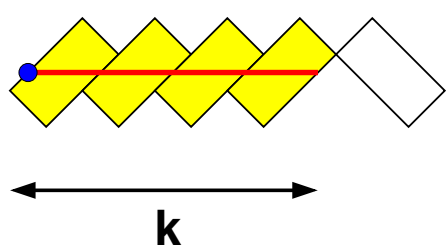

The generating polynomial for the type $m=1,2$ model:

$$
T_{4}^{(m)}\left(\mathcal{A}_{n} ; \tau\right):=\sum_{k=0}^{n-1} \tau^{k} T_{4, k}^{(m)}\left(\mathcal{A}_{n}\right)
$$

is interpreted as the partition functions for non-intersecting restricted Schröder path configurations with some extra weight:

Type 1: $\tau$ per horizontal step taken at vertical position $y=n$ and $\tau$ for a possible diagonal step from $y=n-1$ to $y=n$.

Type 2: $\tau$ per horizontal step taken at vertical position $y=n$.

Theorem 5. The partition functions $T_{4}^{(m)}\left(\mathcal{A}_{n} ; \tau\right)$ for type $m$ refined quarter-turn symmetric domino tilings of the domain $\mathcal{A}_{n}$ are given by:

$$
\begin{aligned}
& T_{4}^{(1)}\left(\mathcal{A}_{n} ; \tau\right)=\operatorname{det}_{0 \leqslant i, j \leqslant n-1}\left(\begin{array}{ll}
\left.\left(\frac{1}{1-r s}+\frac{2 r}{(1-r)(1-r-s-r s)}\right)\right|_{r^{i} s^{j}} & j \leqslant n-2 \\
\left.\left(\frac{1}{1-r s}+\frac{2 \tau r}{(1-\tau r)(1-r-s-r s)}\right)\right|_{r^{i} s^{j}} & j=n-1
\end{array}\right) \\
& =\underset{0 \leqslant i, j \leqslant n-1}{\operatorname{det}}\left(\left(\frac{1}{1-r s}+\frac{2 r}{(1-r)(1-r-s-r s)}\right.\right. \\
& \left.\left.+s^{n-1} r\left\{\frac{2 \tau}{1-\tau r}-\frac{2}{1-r}\right\} \frac{(1+r)^{n-1}}{(1-r)^{n}}\right)\left.\right|_{r^{i} s^{j}}\right) \text {. }
\end{aligned}
$$




$$
\begin{aligned}
& T_{4}^{(2)}\left(\mathcal{A}_{n} ; \tau\right)=\operatorname{det}_{0 \leqslant i, j \leqslant n-1}\left(\begin{array}{ll}
\left.\left(\frac{1}{1-r s}+\frac{2 r}{(1-r)(1-r-s-r s)}\right)\right|_{r^{i} s^{j}} & j \leqslant n-2 \\
\left.\left(\frac{1}{1-r s}+\frac{(1+\tau) r}{(1-\tau r)(1-r-s-r s)}\right)\right|_{r^{i} s^{j}} & j=n-1
\end{array}\right) \\
& =\underset{0 \leqslant i, j \leqslant n-1}{\operatorname{det}}\left(\left(\frac{1}{1-r s}+\frac{2 r}{(1-r)(1-r-s-r s)}\right.\right. \\
& \left.\left.+s^{n-1} r\left\{\frac{1+\tau}{1-\tau r}-\frac{2}{1-r}\right\} \frac{(1+r)^{n-1}}{(1-r)^{n}}\right)\left.\right|_{r^{i} s^{j}}\right) .
\end{aligned}
$$

To prove the theorem, we may evaluate $T_{4}^{(m)}\left(\mathcal{A}_{n} ; \tau\right)$ by use of the LGV formula, by noticing that only the paths ending at $(x, y)=(0, j+1)$ with $j=n-1$ receive a modified weight. More precisely, the partition function for a restricted Schröder path from $(i, 0)$ to $(0, n)$ in types $m=1,2$ correspond to the coefficient of $s^{j}=s^{n-1}$ in the generating functions:

$$
\begin{array}{ll}
\text { Type1 : } & \frac{1}{s}\left\{\left(\frac{\tau r}{1-\tau r} s+\frac{1}{1-\tau r} \tau r s\right) S(r, s)\right\}=\frac{2 \tau r}{1-\tau r} \frac{1}{1-r-s-r s} \\
\text { Type2 : } & \frac{1}{s}\left\{\left(r s+\frac{\tau r}{1-\tau r}(s+r s)\right) S(r, s)\right\}=\frac{(1+\tau) r}{1-\tau r} \frac{1}{1-r-s-r s}
\end{array}
$$

In type 1, we have performed a decomposition of the path according to its last visit at height $n-1$ : it is either followed by an up step (generated by $s$ ) and then by an arbitrary succession of $k$ horizontal steps, with $k \geqslant 1$ since the up step cannot take place at $x=0$ for a restricted Schröder path (generated by $\tau r /(1-\tau r)$ ) or it is followed by a diagonal step from $y=n-1$ to $y=n$ (generated by $\tau r s$ ) and then by an arbitrary succession of $k \geqslant 0$ horizontal steps at $y=n$ (generated by $1 /(1-\tau r)$ ), all of which are preceded by a standard Schröder path, generated by $S(r, s)$. As before, the $1 / s$ global prefactor comes from our choice of extracting the coefficient of $s^{j}=s^{n-1}$ instead of the natural one of $s^{j+1}$.

In type 2, this is again obtained by decomposing the path according its last step(s): it is either a single diagonal step (generated by $r s$ ) or an arbitrary succession of $k \geqslant 1$ left steps (generated by $\tau r /(1-\tau r))$ preceded by either an up or a diagonal step (generated by $s+r s)$ and the preceding part of the path is a generic Schröder path, generated by $S(r, s)$.

The total partition functions $\tilde{S}_{i, n}^{(m)}(\tau)$ for a restricted Schröder path from $(i, 0)$ to $(0, n)$ in type $m=1,2$ read:

$$
\begin{aligned}
& \tilde{S}_{i, n}^{(1)}(\tau)=\left.\frac{2 \tau r}{1-\tau r} \frac{1}{1-r-s-r s}\right|_{r^{i} s^{n-1}}=\left.\frac{2 \tau r}{1-\tau s} \frac{(1+r)^{n-1}}{(1-r)^{n}}\right|_{r^{i}} \\
& \tilde{S}_{i, n}^{(2)}(\tau)=\left.\frac{(1+\tau) r}{1-\tau r} \frac{1}{1-r-s-r s}\right|_{r^{i} s^{n-1}}=\left.\frac{(1+\tau) r}{1-\tau r} \frac{(1+r)^{n-1}}{(1-r)^{n}}\right|_{r^{i}} .
\end{aligned}
$$

Note that the partition functions for all the other paths (ending at vertical coordinate $y \leqslant n-1)$ are the same as before, namely equal to $\tilde{S}_{i, j}$ for paths from $(i, 0)$ to $(0, j)$. 
Applying again the LGV formula, we see that the partition function $T_{4}^{(m)}\left(\mathcal{A}_{n} ; \tau\right)$ is the determinant of a matrix with an analogous form $\mathbb{I}_{n}+M_{n}^{(m)}(\tau)$, where the $n \times n$ matrix $M_{n}^{(m)}(\tau)$ differs from $M_{n}$ only in its last column, in which the entries $\tilde{S}_{i, n}$ are replaced by the new partition functions $\tilde{S}_{i, n}^{(m)}(\tau)$. We deduce the following:

$$
T_{4}^{(m)}\left(\mathcal{A}_{n} ; \tau\right)=\operatorname{det}\left(\mathbb{I}_{n}+M_{n}^{(m)}(\tau)\right), \quad M_{n}^{(m)}(\tau)_{i, j}=\left\{\begin{array}{cc}
\tilde{S}_{i, j+1} & \text { for } j \in[0, n-2] \\
\tilde{S}_{i, n}^{(m)}(\tau) & \text { for } j=n-1
\end{array}\right.
$$

which is nothing but Theorem 5 in its first form.

To put this result in the second and more compact form of Theorem 5 , let us compute the generating function $\left.T_{4}^{(m)}(r, s ; \tau)=\sum_{i, j \geqslant 0} r^{i} s^{j}\left(\mathbb{I}+M^{(m)}(\tau)\right)_{i, j}\right)$ for the new infinite matrix whose $n \times n$ truncations' determinant yields the polynomial $T_{4}^{(m)}\left(\mathcal{A}_{n} ; \tau\right)$. We have

\section{Lemma 6.}

$$
\begin{aligned}
& T_{4}^{(1)}(r, s ; \tau)=\frac{1}{1-r s}+\frac{2 r}{(1-r)(1-r-s-r s)}+s^{n-1} r \frac{2(\tau-1)}{1-\tau r} \frac{(1+r)^{n-1}}{(1-r)^{n+1}} \\
& T_{4}^{(2)}(r, s ; \tau)=\frac{1}{1-r s}+\frac{2 r}{(1-r)(1-r-s-r s)}+s^{n-1} r \frac{\tau-1}{1-\tau r} \frac{(1+r)^{n}}{(1-r)^{n+1}}
\end{aligned}
$$

Proof. To get the new generating function from $T_{4}(r, s)$ in $(4.2)$, we must subtract the contribution of the last column, i.e. $s^{n-1}$ times the coefficient of $s^{n-1}$ in $2 r /((1-r)(1-$ $r-s-r s)$ ) and add the new generating function $s^{n-1} \sum_{i \geqslant 0} \tilde{S}_{i, n}^{(m)}(\tau) r^{i}$. Note that any term of order $\geqslant n$ in $r$ or $s$ is irrelevant and may therefore be chosen arbitrarily, as it does not affect the truncation to size $n$. The net result is the generating function:

$$
\begin{aligned}
& T_{4}^{(1)}(r, s ; \tau)=\frac{1}{1-r s}+\frac{2 r}{(1-r)(1-r-s-r s)}+s^{n-1} r\left\{\frac{2 \tau}{1-\tau r}-\frac{2}{1-r}\right\} \frac{(1+r)^{n-1}}{(1-r)^{n}} \\
& T_{4}^{(2)}(r, s ; \tau)=\frac{1}{1-r s}+\frac{2 r}{(1-r)(1-r-s-r s)}+s^{n-1} r\left\{\frac{1+\tau}{1-\tau r}-\frac{2}{1-r}\right\} \frac{(1+r)^{n-1}}{(1-r)^{n}}
\end{aligned}
$$

and the Lemma follows.

Theorem 5 allows for a very efficient calculation of the partition functions $T_{4}^{(m)}\left(\mathcal{A}_{n} ; \tau\right)$. The first few terms read as follows.

For type 1 , the polynomials $T_{4}^{(1)}\left(\mathcal{A}_{n} ; \tau\right)$ for $n=1, \ldots, 7$ read:

$$
\begin{aligned}
& 1 \\
& 1+2 \tau \\
& 3+14 \tau+6 \tau^{2} \\
& 23+198 \tau+166 \tau^{2}+46 \tau^{3} \\
& 433+6322 \tau+7874 \tau^{2}+4210 \tau^{3}+866 \tau^{4} \\
& 19705+468866 \tau+777258 \tau^{2}+606026 \tau^{3}+240578 \tau^{4}+39410 \tau^{5}
\end{aligned}
$$




$$
\begin{aligned}
2151843+81652574 \tau+169682406 \tau^{2}+172604734 \tau^{3}+99699558 \tau^{4} \\
+31601534 \tau^{5}+4303686 \tau^{6}
\end{aligned}
$$

For type 2 , the polynomials $T_{4}^{(2)}\left(\mathcal{A}_{n} ; \tau\right)$ for $n=1, \ldots, 7$ read:

$$
\begin{aligned}
& 1 \\
& 2+\tau \\
& 10+10 \tau+3 \tau^{2} \\
& 122+182 \tau+106 \tau^{2}+23 \tau^{3} \\
& 3594+7098 \tau+6042 \tau^{2}+2538 \tau^{3}+433 \tau^{4} \\
& 254138+623062 \tau+691642 \tau^{2}+423302 \tau^{3}+139994 \tau^{4}+19705 \tau^{5} \\
& 42978130+125667490 \tau+171143570 \tau^{2}+136152146 \tau^{3}+65650546 \tau^{4} \\
& +17952610 \tau^{5}+2151843 \tau^{6}
\end{aligned}
$$

We note the identities for $n \geqslant 1$

$T_{4}^{(1)}\left(\mathcal{A}_{n} ; 0\right)=T_{4}\left(\mathcal{A}_{n-1}\right),\left.\quad T_{4}^{(1)}\left(\mathcal{A}_{n} ; \tau\right)\right|_{\tau^{n-1}}=2 T_{4}\left(\mathcal{A}_{n-1}\right),\left.\quad T_{4}^{(2)}\left(\mathcal{A}_{n} ; \tau\right)\right|_{\tau^{n-1}}=T_{4}\left(\mathcal{A}_{n-1}\right)$.

All these identities have an easy explanation in terms of paths, which we leave as an exercise for the reader.

\section{Proof of the equivalence between 20V-DWBC1,2 and holey square tilings}

\subsection{From the Izergin-Korepin to the Gessel-Viennot determinant}

The aim of this Section is to prove the identity:

Theorem 7. The number of configurations for the $20 \mathrm{~V}$ model with DWBC1 or DWBC2 on an $n \times n$ grid is equal to that of the quarter-turn symmetric domino tilings of the domain $\mathcal{A}_{n}$, namely:

$$
Z^{20 V}(n)=T_{4}\left(\mathcal{A}_{n}\right)
$$

The proof goes as follows. From Theorem 1, we may get $Z^{20 V}(n)$ from $Z_{[1, \sqrt{2}, 1]}^{6 V}(n)$ whose expression may itself be obtained from the general Izergin-Korepin determinant expression (3.9). Here however, we need to take as spectral parameters the specific values given by (3.8) and, for such homogeneous values, the expression (3.9) cannot be used as such as both the determinant in the expression and the denominator of its prefactor vanish identically, resulting in an indeterminate limit. Some manipulations on the Izergin-Korepin determinant are therefore required before letting $z_{i}$ and $w_{j}$ tend to their homogeneous limiting values $z$ and $w$. Remarkably, the result of these manipulations is a new expression for $Z_{[1, \sqrt{2}, 1]}^{6 V}(n)$ which resembles the Gessel-Viennot determinant encountered in Theorem 4 for the expression of $T_{4}\left(\mathcal{A}_{n}\right)$. The identity $(5.1)$ is then proved by simple rearrangements of the determinant. 
In the limit $z_{i} \rightarrow z$ and $w_{j} \rightarrow w$ for the weights (3.7), the expression (3.9) may be rewritten as:

$$
\begin{aligned}
Z^{6 V}= & (-1)^{\frac{n(n-1)}{2}}\left(\left(q^{2}-q^{-2}\right) \sqrt{z w}\right)^{n}\left((z-w)\left(q^{-2} z-q^{2} w\right)\right)^{n^{2}} \frac{q^{2 n}}{\left(1-q^{4}\right)^{n} w^{n}} \\
& \times \operatorname{det}_{1 \leqslant i, j \leqslant n}\left(\left.\left(\frac{1}{(z+r)-(w+s)}-\frac{1}{(z+r)-q^{4}(w+s)}\right)\right|_{r^{i-1} s^{j-1}}\right) .
\end{aligned}
$$

The passage from (3.9) to (5.2) is explained in [BDFZJ12] and we reproduce the various steps of the computation in Appendix A.

In the particular case where $q, z$ and $w$ take the values (3.8), this leads immediately to

$$
\begin{aligned}
Z_{[1, \sqrt{2}, 1]}^{6 V}(n) & =(-1)^{\frac{n(n-1)}{2}} \frac{\sqrt{2}^{n^{2}}}{\left(\frac{1+\mathrm{i}}{\sqrt{2}}\right)^{n}} \operatorname{det}_{1 \leqslant i, j \leqslant n}\left(\left.\left(\frac{1}{1+r-s}-\frac{1}{1+\mathrm{i}+r-\mathrm{i} s}\right)\right|_{r^{i-1} s^{j-1}}\right) \\
& =\frac{(\mathrm{i} \sqrt{2})^{n(n-1)}}{\left(\frac{1+\mathrm{i}}{2}\right)^{n}} \operatorname{det}_{1 \leqslant i, j \leqslant n}\left(\left.\left(\frac{1}{1+r-s}-\frac{1}{1+\mathrm{i}+r-\mathrm{i} s}\right)\right|_{r^{i-1} s^{j-1}}\right) \\
& =\operatorname{det}_{1 \leqslant i, j \leqslant n}\left(\left.\left(\frac{1-\mathrm{i}}{1+\mathrm{i} \sqrt{2}(r-s)}-\frac{1-\mathrm{i}}{1+\mathrm{i}+\mathrm{i} \sqrt{2}(r-\mathrm{i} s)}\right)\right|_{r^{i-1} s^{j-1}}\right)
\end{aligned}
$$

expressing $Z_{[1, \sqrt{2}, 1]}^{6 V}(n)$ as the determinant of the finite truncation of an infinite matrix with the generating function $f(r, s)$ explicited above. To go from the second to the third line, we used the identity $\left.f(\alpha r, \beta s)\right|_{r^{i-1} s^{j-1}}=\left.\alpha^{i-1} \beta^{j-1} f(r, s)\right|_{r^{i-1} s^{j-1}}$ with $\alpha=\beta=\mathrm{i} \sqrt{2}$ and $\prod_{i, j=1}^{n} \alpha^{i-1} \beta^{j-1}=(\alpha \beta)^{\frac{n(n-1)}{2}}$ to insert the numerator of the prefactor inside the determinant. As for the denominator, we also transferred it inside the determinant via the trivial identity $1 /((1+i) / 2)=1-i$. Performing the transformation

$$
r \rightarrow \frac{1+\mathrm{i}}{\sqrt{2}} \frac{r}{1-\mathrm{i} r}, \quad s \rightarrow \frac{1-\mathrm{i}}{\sqrt{2}} \frac{s}{1+\mathrm{i} s}
$$

in the above infinite matrix generating function leaves the determinant unchanged. Indeed, this amounts to first multiply $r$ by $\alpha=\frac{1+\mathrm{i}}{\sqrt{2}}$ and $s$ by $\beta=\frac{1-\mathrm{i}}{\sqrt{2}}$ changing the determinant by an overall multiplicative factor $\left(\frac{1+\mathrm{i}}{\sqrt{2}} \times \frac{1-\mathrm{i}}{\sqrt{2}}\right)^{\frac{n(n-1)}{2}}=1$ and to then to change $r \rightarrow r /(1-\mathrm{i} r)$ and $s \rightarrow s /(1+\mathrm{i} s)$. Using

$$
\left.f\left(\frac{r}{1-\gamma r}, s\right)\right|_{r^{k} s^{l}}=\left.f(r, s)\right|_{r^{k} s^{l}}+\left.\sum_{m<k} \gamma^{k-m}\left(\begin{array}{c}
k-1 \\
k-m
\end{array}\right) f(r, s)\right|_{r^{m} s^{l}}
$$

the change $r \rightarrow r /(1-\mathrm{i} r)$ amounts to add to each row of the determinant a linear combination of the previous rows while the change $s \rightarrow s /(1+\mathrm{i} s)$ amounts to add to each 
column a linear combination of the previous ones. These operations leave the determinant unchanged. Applying the above substitution (5.3), we get the alternative expression

$$
\begin{aligned}
Z_{[1, \sqrt{2}, 1]}^{6 V}(n) & =\operatorname{det}_{1 \leqslant i, j \leqslant n}\left(\left.(1-\mathrm{i} r)(1+\mathrm{i} s)\left(\frac{1-\mathrm{i}}{1-r-s-r s}+\frac{\mathrm{i}}{1-r s}\right)\right|_{r^{i-1} s^{j-1}}\right) \\
& =\operatorname{det}_{1 \leqslant i, j \leqslant n}\left(\left.\left(\frac{1-\mathrm{i}}{1-r-s-r s}+\frac{\mathrm{i}}{1-r s}\right)\right|_{r^{i-1} s^{j-1}}\right) .
\end{aligned}
$$

Here again the prefactor $(1-\mathrm{i} r)(1+\mathrm{i} s)$ was removed without changing the determinant as the matrices with and without this prefactor are obtained from one another by subtracting from each row (for the $r$-dependent factor) or respectively adding to each column (for the $s$-dependent factor) i times the preceding one.

Using the identity

$$
\begin{aligned}
(1+\mathrm{i} r)(1-s) & \left(\frac{1-\mathrm{i}}{1-r-s-r s}+\frac{\mathrm{i}}{1-r s}\right) \\
= & (1-r)(1-\mathrm{i} s)\left(\frac{1}{1-r s}+\frac{2 r}{(1-r)(1-r-s-r s)}\right),
\end{aligned}
$$

we may play once more the same trick and get the alternative expression

$$
\begin{aligned}
Z_{[1, \sqrt{2}, 1]}^{6 V}(n) & =\operatorname{det}_{1 \leqslant i, j \leqslant n}\left(\left.(1+\mathrm{i} r)(1-s)\left(\frac{1-\mathrm{i}}{1-r-s-r s}+\frac{\mathrm{i}}{1-r s}\right)\right|_{r^{i-1} s^{j-1}}\right) \\
& =\operatorname{det}_{1 \leqslant i, j \leqslant n}\left(\left.(1-r)(1-\mathrm{i} s)\left(\frac{1}{1-r s}+\frac{2 r}{(1-r)(1-r-s-r s)}\right)\right|_{r^{i-1} s^{j-1}}\right) \\
& =\operatorname{det}_{1 \leqslant i, j \leqslant n}\left(\left.\left(\frac{1}{1-r s}+\frac{2 r}{(1-r)(1-r-s-r s)}\right)\right|_{r^{i-1} s^{j-1}}\right) .
\end{aligned}
$$

This latter expression is nothing but that (4.3) for $T_{4}\left(\mathcal{A}_{n}\right)$ up to a trivial shift by 1 of the indices $i$ and $j$. This proves the theorem.

\subsection{Refined equivalence}

We now wish to refine the above result and get the interpretation of $\hat{Z}^{20 V_{B C 1}}(\tau)$ and $\hat{Z}^{20 V_{B C 2}}(\tau)$ in the quarter-turn symmetric tiling language. We have the following:

Theorem 8. The refined partition functions for the $20 \mathrm{~V}$-DWBC1,2 model on an $n \times n$ grid are equal to the refined partition functions for type 1 and 2 quarter-turn symmetric domino tilings of the domain $\mathcal{A}_{n}$, namely

$$
\hat{Z}^{20 V_{B C 1}}(\tau)=T_{4}^{(1)}\left(\mathcal{A}_{n} ; \tau\right), \quad \hat{Z}^{20 V_{B C 2}}(\tau)=T_{4}^{(2)}\left(\mathcal{A}_{n} ; \tau\right) .
$$

The remainder of this section is devoted to the proof of this theorem. From equation, (3.2), we may relate $\hat{Z}^{20 V_{B C 1}}(\tau)$ and $\hat{Z}^{20 V_{B C 2}}(\tau)$ to their analogue $\hat{Z}_{[1, \sqrt{2}, 1]}^{6 V}(\sigma)(3.1)$ for the $6 \mathrm{~V}$ model, with $\sigma=\frac{1+\tau}{2}$. As explained in Section 3.2, this latter partition function may 
be obtained by letting $z_{i}$ and $w_{j}$ tend to their special values $z$ and $w$ of (3.8) except for the spectral parameter $w_{n}$ attached to the last column which tends instead to the value $w u$ for some parameter $u$. We therefore have to evaluate the expression (3.9) of the Izergin-Korepin determinant in this limit. This can be done along the same lines as in the previous section: in the limit $z_{i} \rightarrow z, i=1, \ldots, n, w_{j} \rightarrow w, j=1, \ldots, n-1$ and $w_{n} \rightarrow w u$, the expression (3.9) may be rewritten as:

$$
\begin{aligned}
Z^{6 V}= & (-1)^{\frac{n(n-1)}{2}}\left(\left(q^{2}-q^{-2}\right) \sqrt{z w}\right)^{n-1}\left((z-w)\left(q^{-2} z-q^{2} w\right)\right)^{n(n-1)} \frac{q^{2(n-1)}}{\left(1-q^{4}\right)^{n-1} w^{n-1}} \\
& \times\left(q^{2}-q^{-2}\right) \sqrt{z w u}\left((z-w u)\left(q^{-2} z-q^{2} w u\right)\right)^{n} \frac{q^{2}}{\left(1-q^{4}\right) w u} \frac{1}{(w u-w)^{n-1}} \\
& \times \operatorname{det}_{1 \leqslant i, j \leqslant n}\left(\begin{array}{cc}
\left.\left(\frac{1}{(z+r)-(w+s)}-\frac{1}{(z+r)-q^{4}(w+s)}\right)\right|_{r^{i-1} s^{j-1}} & j \leqslant n-1 \\
\left.\left(\frac{1}{(z+r)-w u}-\frac{1}{(z+r)-q^{4} w u}\right)\right|_{r^{i-1}} & j=n
\end{array}\right) .
\end{aligned}
$$

A derivation of this expression in given in Appendix B. For the specific values (3.8), this yields

$$
\begin{aligned}
& Z_{[1, \sqrt{2}, 1] ;\left[\frac{(u+\mathrm{i})(1-\mathrm{i})}{2}, \sqrt{2} \frac{1+u}{2}, \sqrt{u}\right]}^{6 V}(n) \\
& =(-1)^{\frac{n(n-1)}{2}} \frac{(\sqrt{2})^{n^{2}}}{\left(\frac{1+\mathrm{i}}{\sqrt{2}}\right)^{n}} \frac{1}{\sqrt{u}}\left(\frac{1+u}{2} \frac{(u+\mathrm{i})(1-\mathrm{i})}{2}\right)^{n}\left(\frac{1+\mathrm{i}}{1-u}\right)^{n-1} \\
& \times \operatorname{det}_{1 \leqslant i, j \leqslant n}\left(\begin{array}{cc}
\left.\left(\frac{1}{1+r-s}-\frac{1}{1+\mathrm{i}+r-\mathrm{i} s}\right)\right|_{r^{i-1} s^{j-1}} & j \leqslant n-1 \\
\left.\left(\frac{1}{\frac{1-\mathrm{i} u}{1-\mathrm{i}}+r}-\frac{1}{\frac{1+u}{1-\mathrm{i}}+r}\right)\right|_{r^{i-1}} & j=n
\end{array}\right) \\
& =\frac{1}{\sqrt{u}}(\mathrm{i} \sqrt{2})^{n-1}\left(\frac{1+u}{2} \frac{(u+\mathrm{i})(1-\mathrm{i})}{2}\right)^{n}\left(\frac{1+\mathrm{i}}{1-u}\right)^{n-1} \\
& \times \operatorname{det}_{1 \leqslant i, j \leqslant n}\left(\begin{array}{cc}
\left.\left(\frac{1-\mathrm{i}}{1+\mathrm{i} \sqrt{2}(r-s)}-\frac{1-\mathrm{i}}{1+\mathrm{i}+\mathrm{i} \sqrt{2}(r-\mathrm{i} s)}\right)\right|_{r^{i-1} s^{j-1}} & j \leqslant n-1 \\
\left.\left(\frac{1-\mathrm{i}}{\frac{1-\mathrm{i} u}{1-\mathrm{i}}+\mathrm{i} \sqrt{2} r}-\frac{1-\mathrm{i}}{\frac{1+u}{1-\mathrm{i}}+\mathrm{i} \sqrt{2} r}\right)\right|_{r^{i-1}} & j=n
\end{array}\right) .
\end{aligned}
$$

Here the notation $Z_{[1, \sqrt{2}, 1] ;\left[\frac{(u+\mathrm{i})(1-\mathrm{i})}{2}, \sqrt{2} \frac{1+u}{2}, \sqrt{u}\right]}^{6}(n)$ indicates that the weights in the last column are different from those in the other columns, with the indicated $u$-dependent values. Again we perform the substitution (5.3). Note that, as opposed to what we had before, the change in $s$ does not affect the last column $j=n$. The effect of the substitution on the determinant is compensated by multiplying simultaneously by an overall factor $\left(\frac{1-i}{\sqrt{2}}\right)^{n-1}$. 
This leads to

$$
\begin{aligned}
& Z_{[1, \sqrt{2}, 1] ;\left[\frac{(u+\mathrm{i})(1-\mathrm{i})}{2}, \sqrt{2} \frac{1+u}{2}, \sqrt{u}\right]}^{(n)} \\
& =\frac{1}{\sqrt{u}}(\mathrm{i} \sqrt{2})^{n-1}\left(\frac{1+u}{2} \frac{(u+\mathrm{i})(1-\mathrm{i})}{2}\right)^{n}\left(\frac{1+\mathrm{i}}{1-u}\right)^{n-1}\left(\frac{1-\mathrm{i}}{\sqrt{2}}\right)^{n-1} \\
& \times \operatorname{det}_{1 \leqslant i, j \leqslant n}\left(\begin{array}{cc}
\left.(1-\mathrm{i} r)(1+\mathrm{i} s)\left(\frac{1-\mathrm{i}}{1-r-s-r s}+\frac{\mathrm{i}}{1-r s}\right)\right|_{r^{i-1} s^{j-1}} & j \leqslant n-1 \\
\left.(1-\mathrm{i} r)\left(\frac{-2 \mathrm{i}}{1-\mathrm{i} u+(\mathrm{i}-u) r}-\frac{-2 \mathrm{i}}{1+u+\mathrm{i}(1-u) r}\right)\right|_{r^{i-1}} & j=n
\end{array}\right) \\
& =\frac{1}{\sqrt{u}}\left(\frac{(1+u)(u+\mathrm{i})(1+\mathrm{i})}{2(1-u)}\right)^{n} \\
& \times \operatorname{det}_{1 \leqslant i, j \leqslant n}\left(\begin{array}{cc}
\left.(1-\mathrm{i} r)(1+\mathrm{i} s)\left(\frac{1-\mathrm{i}}{1-r-s-r s}+\frac{\mathrm{i}}{1-r s}\right)\right|_{r^{i-1} s^{j-1}} & j \leqslant n-1 \\
\left.(1-\mathrm{i} r)\left(\frac{u-1}{1-\mathrm{i} u+(\mathrm{i}-u) r}-\frac{u-1}{1+u+\mathrm{i}(1-u) r}\right)\right|_{r^{i-1}} & j=n
\end{array}\right) \\
& =\frac{1}{\sqrt{u}}\left(\frac{(1+u)(u+\mathrm{i})(1+\mathrm{i})}{2(1-u)}\right)^{n} \operatorname{det}_{1 \leqslant i, j \leqslant n}\left(\begin{array}{cc}
\left.\left(\frac{1-\mathrm{i}}{1-r-s-r s}+\frac{\mathrm{i}}{1-r s}\right)\right|_{r^{i-1}} s^{j-1} & j \leqslant n-1 \\
\left.\left(\frac{u-1}{1-\mathrm{i} u+(\mathrm{i}-u) r}-\frac{u-1}{1+u+\mathrm{i}(1-u) r}\right)\right|_{r^{i-1}} & j=n
\end{array}\right)
\end{aligned}
$$

where we again removed the factors $(1-\mathrm{i} r)$ and $(1+\mathrm{i} s)$ without changing the determinant.

We now recall from (3.5) the expression

$$
\begin{aligned}
Z_{[1, \sqrt{2}, 1] ;\left[\frac{(u+\mathrm{i})(1-\mathrm{i})}{2}, \sqrt{2} \frac{1+u}{2}, \sqrt{u}\right]}^{6 V}(n) & =\sum_{\ell=1}^{n} Z_{[1, \sqrt{2}, 1] ; \ell}^{6 V}\left(\frac{1+u}{2}\right)^{\ell-1} \sqrt{u}\left(\frac{(u+\mathrm{i})(1-\mathrm{i})}{2}\right)^{n-\ell} \\
& =\sqrt{u}\left(\frac{(u+\mathrm{i})(1-\mathrm{i})}{2}\right)^{n-1} \hat{Z}_{[1, \sqrt{2}, 1]}^{6 V}(\sigma)
\end{aligned}
$$

where $\sigma=\frac{1+u}{(\mathrm{i}+u)(1-\mathrm{i})}$, or equivalently, $u=\frac{1-(1+\mathrm{i}) \sigma}{(1-\mathrm{i}) \sigma-1}$. Comparing the two expressions above leads to

$$
\begin{aligned}
& \hat{Z}_{[1, \sqrt{2}, 1]}^{6 V}(\sigma)=\frac{1}{u}\left(\frac{(1+u)(u+\mathrm{i})(1+\mathrm{i})}{2(1-u)}\right)\left(\mathrm{i} \frac{1+u}{1-u}\right)^{n-1} \\
& \times \operatorname{det}_{1 \leqslant i, j \leqslant n}\left(\begin{array}{cc}
\left.\left(\frac{1-\mathrm{i}}{1-r-s-r s}+\frac{\mathrm{i}}{1-r s}\right)\right|_{r^{i-1}} s^{j-1} & j \leqslant n-1 \\
\left.\left(\frac{u-1}{1-\mathrm{i} u+(\mathrm{i}-u) r}-\frac{u-1}{1+u+\mathrm{i}(1-u) r}\right)\right|_{r^{i-1}} & j=n
\end{array}\right) \\
& =\frac{\sigma-1}{\sigma(1-\mathrm{i})+\mathrm{i}}\left(\frac{\sigma}{\sigma-1}\right)^{n} \operatorname{det}_{1 \leqslant i, j \leqslant n}\left(\begin{array}{cc}
\left.\left(\frac{1-\mathrm{i}}{1-r-s-r s}+\frac{\mathrm{i}}{1-r s}\right)\right|_{r^{i-1}}{ }^{j-1} & j \leqslant n-1 \\
\left.\left(\frac{1-\mathrm{i}}{1+(1-2 \sigma) r}+\frac{\mathrm{i}}{\sigma+(1-\sigma) r}\right)\right|_{r^{i-1}} & j=n
\end{array}\right) \text {. }
\end{aligned}
$$

Setting $\sigma=\frac{1+\tau}{2}$ and using (3.2), we deduce alternatively

$$
\begin{aligned}
\hat{Z}^{20 V_{B C 2}}(\tau)= & \frac{(1+\mathrm{i})(\tau-1)}{2(\tau+\mathrm{i})}\left(\frac{\tau+1}{\tau-1}\right)^{n} \operatorname{det}_{1 \leqslant i, j \leqslant n}\left(\begin{array}{cc}
\left.\left(\frac{1-\mathrm{i}}{1-r-s-r s}+\frac{\mathrm{i}}{1-r s}\right)\right|_{r^{i-1} s^{j-1}} & j \leqslant n-1 \\
\left.\left(\frac{1-\mathrm{i}}{1-\tau r}+\frac{2 \mathrm{i}}{(\tau+1)-(\tau-1) r}\right)\right|_{r^{i-1}} & j=n
\end{array}\right) \\
& =\frac{1+\mathrm{i}}{\tau+\mathrm{i}}\left(\frac{\tau+1}{\tau-1}\right)^{n-1} \operatorname{det}\left(Q_{n}+P_{n}\right)
\end{aligned}
$$


where

$$
\begin{aligned}
& \left(P_{n}\right)_{i, j}=\left\{\begin{array}{cc}
\left.\left(\frac{\mathrm{i}}{1-r s}\right)\right|_{r^{i-1} s^{j-1}}=\mathrm{i} \delta_{i, j} & j \leqslant n-1 \\
\left.\left(\frac{\mathrm{i}}{1-\frac{\tau-1}{\tau+1} r}\right)\right|_{r^{i-1}}=\mathrm{i}\left(\frac{\tau-1}{\tau+1}\right)^{i-1} & j=n
\end{array}\right. \\
& \left(Q_{n}\right)_{i, j}=\left\{\begin{array}{cc}
\left.\left(\frac{1-\mathrm{i}}{1-r-s-r s}\right)\right|_{r^{i-1} s^{j-1}} & j \leqslant n-1 \\
\left.\left(\frac{\frac{1-\mathrm{i}}{2}(\tau+1)}{1-\tau r}\right)\right|_{r^{i-1}} & j=n
\end{array}\right.
\end{aligned}
$$

Now the matrix $P_{n}$ differs from the matrix $i \mathbb{I}_{n}$ only in its last column and its determinant is therefore easily obtained as

$$
\operatorname{det}\left(P_{n}\right)=\mathrm{i}^{n-1}\left(P_{n}\right)_{n, n}=\mathrm{i}^{n}\left(\frac{\tau-1}{\tau+1}\right)^{n-1}
$$

and we deduce

$$
\hat{Z}^{20 V_{B C 2}}(\tau)=\frac{1+\mathrm{i}}{\tau+\mathrm{i}} \operatorname{det}\left(\mathrm{i} \mathbb{I}_{n}+\mathrm{i} Q_{n} P_{n}^{-1}\right)
$$

Using

$$
\mathrm{i}\left(P_{n}^{-1}\right)_{i, j}=\left\{\begin{array}{cc}
\delta_{i, j} & j \leqslant n-1 \\
-\left(\frac{\tau+1}{\tau-1}\right)^{n-i} & i \leqslant n-1, j=n \\
\left(\frac{\tau+1}{\tau-1}\right)^{n-1} & i=n, j=n
\end{array},\right.
$$

we get i $\left(Q_{n} P_{n}^{-1}\right)_{i, j}=\left(Q_{n}\right)_{i, j}$ for $j<n$, while

$$
\begin{aligned}
& \mathrm{i}\left(Q_{n} P_{n}^{-1}\right)_{i, n}=\left.\left(\frac{\frac{1-\mathrm{i}}{2}(\tau+1)}{1-\tau r}\right)\right|_{r^{i-1}}\left(\frac{\tau+1}{\tau-1}\right)^{n-1}-\left.\sum_{k=1}^{n-1}\left(\frac{1-\mathrm{i}}{1-r-s-r s}\right)\right|_{r^{i-1} s^{k-1}}\left(\frac{\tau+1}{\tau-1}\right)^{n-k} \\
& =\left.\left(\frac{\tau+1}{\tau-1}\right)^{n-1} \underbrace{\left(\frac{\frac{1-\mathrm{i}}{2}(\tau+1)}{1-\tau r}-\frac{1-\mathrm{i}}{1-r-\frac{\tau-1}{\tau+1}-r \frac{\tau-1}{\tau+1}}\right)}_{=0}\right|_{r^{i-1}} \\
& +\left.\sum_{k=n}^{\infty}\left(\frac{\tau+1}{\tau-1}\right)^{n-k}\left(\frac{1-\mathrm{i}}{1-r-s-r s}\right)\right|_{r^{i-1} s^{k-1}} \\
& =\left.(1-\mathrm{i})\left(\sum_{k=n}^{\infty}\left(\frac{\tau+1}{\tau-1}\right)^{n-k} \frac{(1+r)^{k-1}}{(1-r)^{k}}\right)\right|_{r^{i-1}}=\left.\frac{1-\mathrm{i}}{2}\left(\frac{1+r}{1-r}\right)^{n-1}\left(\frac{1+\tau}{1-\tau r}\right)\right|_{r^{i-1}} .
\end{aligned}
$$

We end up with the expression

$$
\begin{aligned}
& \hat{Z}^{20 V_{B C 2}}(\tau)=\frac{1+\mathrm{i}}{\tau+\mathrm{i}} \\
& \times \operatorname{det}_{1 \leqslant i, j \leqslant n}\left(\left.\left(\frac{\mathrm{i}}{1-r s}+\frac{1-\mathrm{i}}{1-r-s-r s}+s^{n-1}\left(\frac{1+r}{1-r}\right)^{n-1}\left\{\frac{1-\mathrm{i}}{2} \frac{1+\tau}{1-\tau r}-\frac{1-\mathrm{i}}{1-r}\right\}\right)\right|_{r^{i-1} s^{j-1}}\right)
\end{aligned}
$$

where the last term corrects the wrong value $\left.\frac{1-\mathrm{i}}{1-r-s-r s}\right|_{r^{i-1} s^{n-1}}=\left.\left(\frac{1+r}{1-r}\right)^{n-1} \frac{1-\mathrm{i}}{1-r}\right|_{r^{i-1}}$ coming from the second term to the correct value above. As before, we may multiply the function 
inside the determinant by $\frac{(1+\mathrm{i} r)(1-s)}{(1-r)(1-\mathrm{i} s)}$ without changing the value of the determinant. Using again the identity (5.4), we obtain

$$
\begin{aligned}
& \hat{Z}^{20 V_{B C 2}}(\tau)= \frac{1+\mathrm{i}}{\tau+\mathrm{i}} \operatorname{det}_{1 \leqslant i, j \leqslant n}\left(\left(\frac{1}{1-r s}+\frac{2 r}{(1-r)(1-r-s-r s)}\right.\right. \\
&\left.\left.+s^{n-1}\left(\frac{1+r}{1-r}\right)^{n-1} \frac{(1+\mathrm{i} r)(1-\not)}{(1-r)(1-\mathrm{i} \not)}\left(\frac{1-\mathrm{i}}{2} \frac{1+\tau}{1-\tau r}-\frac{1-\mathrm{i}}{1-r}\right)\right)\left.\right|_{r^{i-1} s^{j-1}}\right) \\
&= \frac{1+\mathrm{i}}{\tau+\mathrm{i}} \operatorname{det}_{1 \leqslant i, j \leqslant n}\left(K_{i, j}\right) \\
& K_{i, j}:=\left.\left(\frac{1}{1-r s}+\frac{2 r}{(1-r)(1-r-s-r s)}+s^{n-1} \frac{(1+r)^{n-1}}{(1-r)^{n}} \frac{1+\mathrm{i} r}{1+\mathrm{i}}\left\{\frac{1+\tau}{1-\tau r}-\frac{2}{1-r}\right\}\right)\right|_{r^{i-1} s^{j-1}}
\end{aligned}
$$

where the crossed out $\$$ play no role and were thus removed. In this form, the expression is now very close to that of Theorem 5 for $T_{4,2}\left(\mathcal{A}_{n} ; \tau\right)$, namely (with a trivial shift by 1 of the indices)

$$
\begin{aligned}
& T_{4,2}\left(\mathcal{A}_{n} ; \tau\right)=\operatorname{det}_{1 \leqslant i, j \leqslant n}\left(L_{i, j}\right) \\
& L_{i, j}:=\left.\left(\frac{1}{1-r s}+\frac{2 r}{(1-r)(1-r-s-r s)}+s^{n-1} \frac{(1+r)^{n-1}}{(1-r)^{n}} r\left\{\frac{(1+\tau)}{1-\tau r}-\frac{2}{1-r}\right\}\right)\right|_{r^{i-1} s^{j-1}} .
\end{aligned}
$$

The identification of the two formulas follows from the following simple remark:

Lemma 9. We have the identities

$$
\begin{array}{ll}
K_{i, j}=L_{i, j} & j<n \\
K_{i, n}=\frac{\tau+\mathrm{i}}{1+\mathrm{i}} L_{i, n}+\frac{\tau-1}{1+\mathrm{i}} \sum_{j=1}^{n-1} L_{i, j} & j=n .
\end{array}
$$

Proof. The first statement for $j<n$ is by definition. For $j=n$, we use

$$
L_{i, j}=\left\{\begin{array}{cc}
\left.\left(r^{j-1}+\frac{(1+r)^{j-1}}{(1-r)^{j}} \frac{2 r}{1-r}\right)\right|_{r^{i-1}} & j \leqslant n-1 \\
\left.\left(r^{n-1}+\frac{(1+r)^{n-1}}{(1-r)^{n}} \frac{(1+\tau) r}{1-\tau r}\right)\right|_{r^{i-1}} & j=n
\end{array}\right.
$$


so that

$$
\begin{aligned}
\frac{\tau+\mathrm{i}}{1+\mathrm{i}} L_{i, n}+\frac{\tau-1}{1+\mathrm{i}} \sum_{j=1}^{n-1} L_{i, j}= & \left(\frac{\tau+\mathrm{i}}{1+\mathrm{i}} r^{n-1}+\frac{\tau-1}{1+\mathrm{i}} \frac{1-r^{n-1}}{1-r}\right. \\
& \left.+\frac{\tau+\mathrm{i}}{1+\mathrm{i}} \frac{(1+r)^{n-1}}{(1-r)^{n}} \frac{(1+\tau) r}{1-\tau r}-\frac{\tau-1}{1+\mathrm{i}} \frac{1-\left(\frac{1+r}{1-r}\right)^{n-1}}{1-r}\right)\left.\right|_{r^{i-1}} \\
= & \left.\left(\left(\frac{\tau+\mathrm{i}}{1+\mathrm{i}}-\frac{\tau-1}{1+\mathrm{i}} \frac{1}{1-\not r}\right) r^{n-1}+\left(\frac{\tau+\mathrm{i}}{1+\mathrm{i}} \frac{(1+\tau) r}{1-\tau r}+\frac{\tau-1}{1+\mathrm{i}}\right) \frac{(1+r)^{n-1}}{(1-r)^{n}}\right)\right|_{r^{i-1}} \\
= & \left.\left(r^{n-1}+\left(\frac{\tau+\mathrm{i}}{1+\mathrm{i}} \frac{(1+\tau) r}{1-\tau r}+\frac{\tau-1}{1+\mathrm{i}}\right) \frac{(1+r)^{n-1}}{(1-r)^{n}}\right)\right|_{r^{i-1}} \\
= & \left.\left(r^{n-1}+\left(\frac{1+\mathrm{i} r}{1+\mathrm{i}}\left(\frac{1+\tau}{1+\tau r}-\frac{2}{1-r}\right)+\frac{2 r}{1-r}\right) \frac{(1+r)^{n-1}}{(1-r)^{n}}\right)\right|_{r^{i-1}}=K_{i, n} .
\end{aligned}
$$

Here again, we removed the crossed out $\not r$ as it plays no role for $i \leqslant n$ and we used the easily checked identity

$$
\frac{\tau+\mathrm{i}}{1+\mathrm{i}} \frac{(1+\tau) r}{1-\tau r}+\frac{\tau-1}{1+\mathrm{i}}=\frac{1+\mathrm{i} r}{1+\mathrm{i}}\left(\frac{1+\tau}{1+\tau r}-\frac{2}{1-r}\right)+\frac{2 r}{1-r} .
$$

The lemma follows.

With the identities (5.8), the expression (5.6) is transformed into (5.7) by a simple expansion of the determinant with respect to the last column. This completes the proof that the expression (5.6) for $\hat{Z}^{20 V_{B C 2}}(\tau)$ matches that $(5.7)$ for $T_{4}^{(2)}\left(\mathcal{A}_{n} ; \tau\right)$. This amounts precisely to the second statement of Theorem 8 .

To compute $\hat{Z}^{20 V_{B C 1}}(\tau)$, we first note that the expression (5.7) for $\hat{Z}^{20 V_{B C 2}}(\tau)$ can be substituted in (3.2) to get

$$
\hat{Z}_{[1, \sqrt{2}, 1]}^{6 V}(\sigma)=\operatorname{det}_{1 \leqslant i, j \leqslant n}\left(\begin{array}{cc}
\left.\left(\frac{1}{1-r s}+\frac{2 r}{(1-r)(1-r-s-r s)}\right)\right|_{r^{i-1} s^{j-1}} & j \leqslant n-1 \\
\left.\left(\frac{1}{1-r s}+\frac{2 \sigma r}{(1-(2 \sigma-1) r)(1-r-s-r s)}\right)\right|_{r^{i-1} s^{j-1}} & j=n
\end{array}\right)
$$

for $\sigma=\frac{1+\tau}{2}$. The partition function $\hat{Z}^{20 V_{B C 1}}(\tau)$ is then obtained via (3.3), which yields

$$
\begin{aligned}
\hat{Z}^{20 V_{B C 1}}(\tau)= & \frac{2 \tau}{1+\tau} \operatorname{det}_{1 \leqslant i, j \leqslant n}\left(\begin{array}{cc}
\left.\left(\frac{1}{1-r s}+\frac{2 r}{(1-r)(1-r-s-r s)}\right)\right|_{r^{i-1} s^{j-1}} & j \leqslant n-1 \\
\left.\left(\frac{1}{1-r s}+\frac{(1+\tau) r}{(1-\tau r)(1-r-s-r s)}\right)\right|_{r^{i-1} s^{j-1}} & j=n
\end{array}\right) \\
& +\frac{1-\tau}{1+\tau} \operatorname{det}_{1 \leqslant i, j \leqslant n}\left(\begin{array}{cc}
\left.\left(\frac{1}{1-r s}+\frac{2 r}{(1-r)(1-r-s-r s)}\right)\right|_{r^{i-1} s^{j-1}} & j \leqslant n-1 \\
\left.\left(\frac{1}{1-r s}\right)\right|_{r^{i-1} s^{j-1}} & j=n
\end{array}\right) \\
= & \operatorname{det}_{1 \leqslant i, j \leqslant n}\left(\begin{array}{cc}
\left.\left(\frac{1}{1-r s}+\frac{2 r}{(1-r)(1-r-s-r s)}\right)\right|_{r^{i-1} s^{j-1}} & j \leqslant n-1 \\
\left.\left(\frac{2 \tau}{1+\tau}\left(\frac{1}{1-r s}+\frac{(1+\tau) r}{(1-\tau r)(1-r-s-r s)}\right)+\frac{1-\tau}{1+\tau} \frac{1}{1-r s}\right)\right|_{r^{i-1} s^{j-1}} & j=n
\end{array}\right)
\end{aligned}
$$



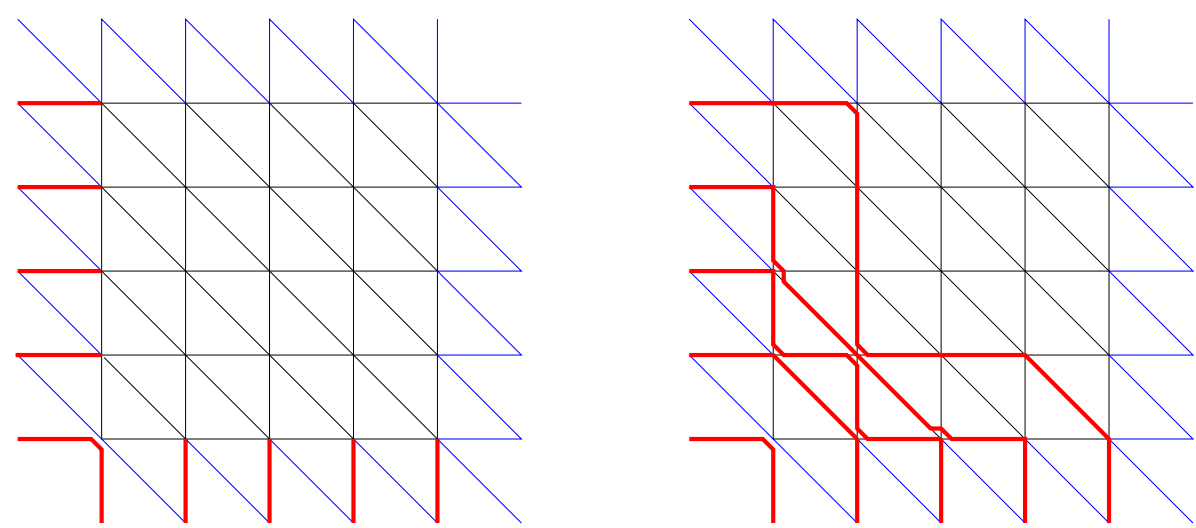

Figure 17: Left: DWBC3 boundary conditions for an $n \times n$ grid for $n=5$. All outer horizontal edges along the West boundary, as well as all vertical edges along the South boundary are occupied by paths. All other outer edges are empty. Right: This gives rise to configurations of $n=5$ non-intersecting osculating Schröder paths, such as that depicted.

$$
=\operatorname{det}_{1 \leqslant i, j \leqslant n}\left(\begin{array}{cc}
\left.\left(\frac{1}{1-r s}+\frac{2 r}{(1-r)(1-r-s-r s)}\right)\right|_{r^{i-1} s^{j-1}} & j \leqslant n-1 \\
\left.\left(\frac{1}{1-r s}+\frac{2 \tau r}{(1-\tau r)(1-r-s-r s)}\right)\right|_{r^{i-1} s^{j-1}} & j=n
\end{array}\right) .
$$

We recognize the expression of Theorem 5 for $T_{4}^{(1)}\left(\mathcal{A}_{n} ; \tau\right)$ (up to a trivial shift in the indices) so that the first statement of Theorem 8 follows.

\section{Other boundary conditions}

In this section, we explore other possible DWBC-like boundary conditions. One of them, which we call DWBC3, leads to a striking conjecture.

\subsection{The 20V model with DWBC3}

We consider the following variant of the DWBC1,2 of Section 2 for the $20 \mathrm{~V}$ model. We still consider a square grid of size $n \times n$ in the square lattice with the second diagonal edge on each face. The boundary conditions on the external edge orientations are now as follows: (i) all horizontal external edges point towards the square domain (ii) all vertical external edges point away from the square domain (iii) all diagonal external edges points towards the NW.

In the osculating Schröder path formulation, we have paths entering the grid on each horizontal external edge on the West boundary, and exiting the grid on each vertical external edge along the South boundary (see Fig. 17 for an illustration).

The 20V-DWBC3 configurations are easily counted by use of transfer matrices, giving rise to the sequence $B_{n}$ of (1.2). Remarkably, these numbers appear in the context of yet 
another enumeration problem of domino tilings, which we describe now.

\subsection{Domino tilings of a triangle and the DWBC3 conjecture}

\subsubsection{Domino tilings of a square}

Let us consider the number of tilings of a $(2 n) \times(2 n)$ square domain $\mathcal{S}_{n}$ of the square lattice by means of rectangular dominos of size $2 \times 1$ and $1 \times 2$. This is part of the archetypical dimer problems solved by Kasteleyn and Temperley and Fisher [Kas63, TF61]. If $T\left(\mathcal{S}_{n}\right)$ denotes this number, we have:

$$
T\left(\mathcal{S}_{n}\right)=\prod_{i=1}^{n} \prod_{j=1}^{n}\left\{4 \cos ^{2}\left(\frac{i}{2 n+1}\right)+4 \cos ^{2}\left(\frac{j}{2 n+1}\right)\right\} .
$$

It was later observed that:

$$
T\left(\mathcal{S}_{n}\right)=2^{n} b_{n}^{2}
$$

with

$$
b_{n}=\prod_{1 \leqslant i<j \leqslant n}\left\{4 \cos ^{2}\left(\frac{i}{2 n+1}\right)+4 \cos ^{2}\left(\frac{j}{2 n+1}\right)\right\}=1,3,29,901, \ldots
$$

where we recognize the first terms of the sequence $B_{n}(1.2)$ above. The asymptotics of the numbers $b_{n}$ for large $n$ read:

$$
\lim _{n \rightarrow \infty} \frac{1}{n^{2}} \log \left(b_{n}\right)=\frac{2}{\pi} G=.583121808 \ldots,
$$

where $G$ is the Catalan constant, $G=1-\frac{1}{3^{2}}+\frac{1}{5^{2}}-\frac{1}{7^{2}}+\cdots$.

\subsubsection{Domino tilings of a triangle}

A combinatorial proof of the integrality of $b_{n}$ due to Pachter [Pac97] shows in fact that the sequence $b_{n}$ enumerates domino tilings of a "triangle" $\mathcal{T}_{n}$ (half of the square $\mathcal{S}_{n}$ ), with the following shape of an inverted staircase with the first step of size 1 , and all other steps of size 2 :

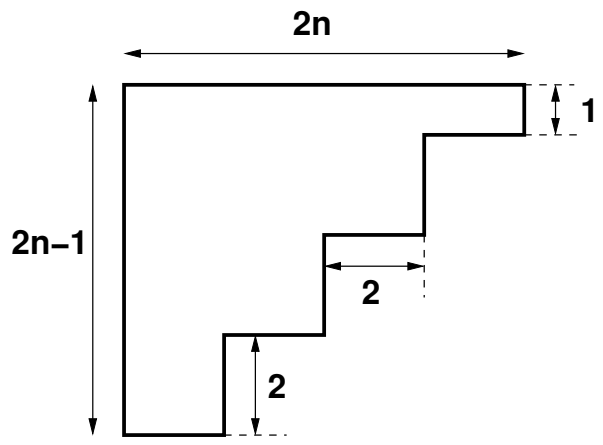


In our notations, we write ${ }^{6}$ :

$$
b_{n}=T\left(\mathcal{T}_{n}\right)
$$

From a practical point of view, it is interesting to recover the first terms of the sequence $b_{n}=T\left(\mathcal{T}_{n}\right)$ from standard combinatorial techniques easily amenable to generalizations. As before, the enumeration of domino tilings of $\mathcal{T}_{n}$ is easily performed by means of nonintersecting Schröder paths, now with steps $(1,1),(1,-1)$ and $(2,0)$ as shown below:

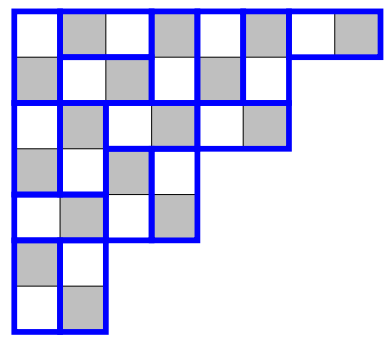

(a)

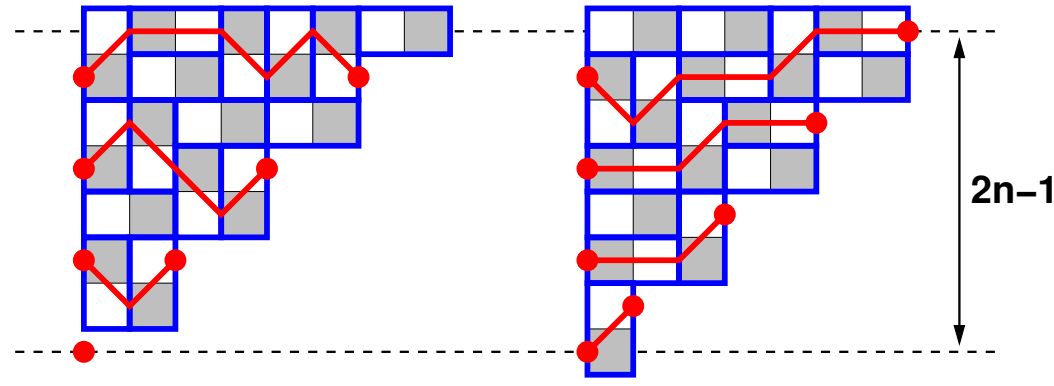

(c)

Here we have first bi-colored the underlying square lattice, considered a tiling configuration (a), and used the dictionary (4.1) mapping each domino to a path step. We have displayed two equivalent path formulations (b) and (c) of the same domain (upon reflection and rotation by $90^{\circ}$ ). In both cases, the paths are non-intersecting Schröder paths with fixed ends as shown, and constrained to remain within a strip of height $2 n-1$ (we have added a trivial path of length 0 in the case (b) for simplicity).

The path configurations are best enumerated by means of the LGV formula. Let $S_{a, b}^{(L)}(M)$ denote the partition function of a single Schröder path (with steps $(1,1),(1,-1)$ and $(2,0))$, starting at point $(0, a)$, ending at point $(M, b)$ and constrained to remain in the strip $0 \leqslant y \leqslant L$. Then the partition function for domino tilings of $\mathcal{T}_{n}$ is:

$$
T\left(\mathcal{T}_{n}\right)=\operatorname{det}_{0 \leqslant i, j \leqslant n-1}\left(S_{2 i, 2 j}^{(2 n-1)}(2 j)\right)=\operatorname{det}_{0 \leqslant i, j \leqslant n-1}\left(S_{2 i, 2 j+1}^{(2 n-1)}(2 j+1)\right)
$$

corresponding respectively to situations (b) and (c).

The single path partition function $S_{a, b}^{(L)}(M)$ may easily be generated from the following recursion relation:

$$
S_{a, b}^{(L)}(M)=\left\{\begin{array}{c}
0 \text { if } a<0 \text { or } a>L \text { or } b<0 \text { or } b>L \\
S_{a, b}^{(L)}(M-2)+S_{a-1, b}^{(L)}(M-1)+S_{a+1, b}^{(L)}(M-1) \text { otherwise }
\end{array}\right.
$$

together with the initial conditions $S_{a, b}^{(L)}(-1)=0$ and $S_{a, b}^{(L)}(0)=\delta_{a, b}$ when $a, b \in[0, L]$ and 0 otherwise. This allows to recover the first terms of the sequence $b_{n}$.

\footnotetext{
${ }^{6}$ This formula can also be obtained by using Ciucu's factorization theorem (Theorem 1.2 in [Ciu97] - see more particularly Theorem 2.1 and Figure 2.2 in this paper).
} 

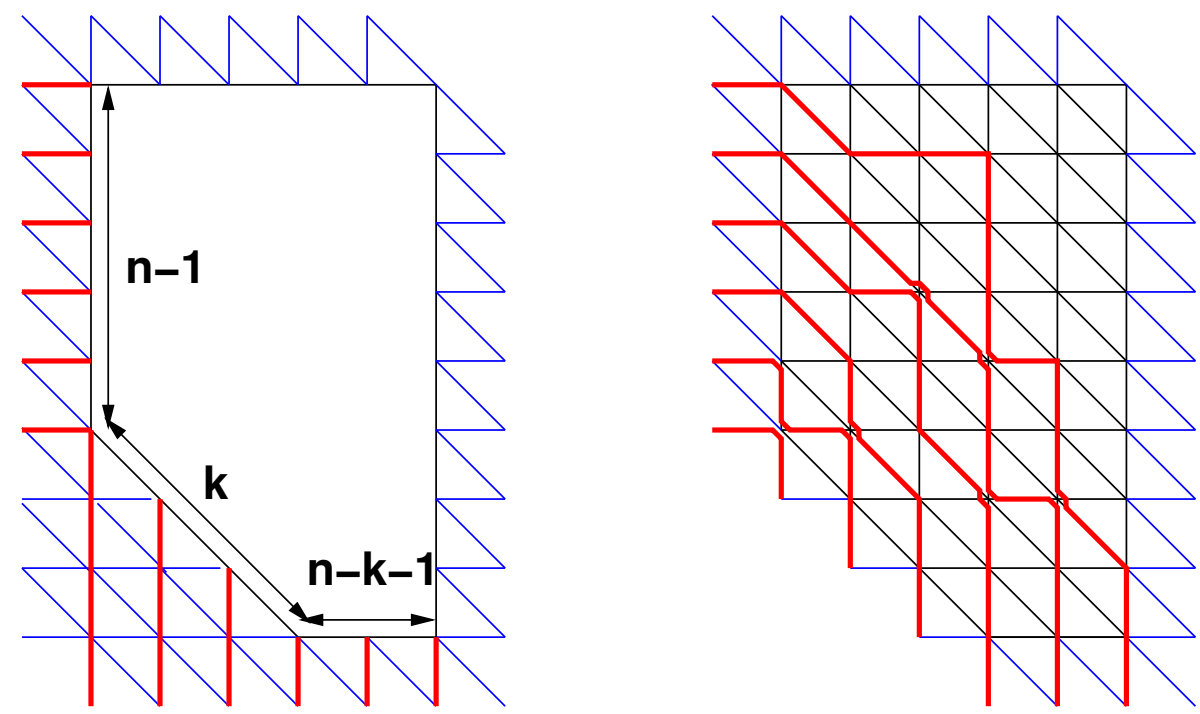

Figure 18: Left: Extension of the DWB3 boundary on a $(n+k) \times n$ rectangular grid in osculating Schröder path representation: due to non-intersection constraints the effective domain is a pentagon of shape $(n-1) \times k \times(n-k-1) \times(n+k-1) \times(n-1)$. Right: A sample path configuration.

\subsubsection{The DWBC3 conjecture}

In view of the matching of the first values of $B_{n}$ with the sequence $b_{n}$, we are led to the following:

Conjecture 10. We conjecture that the number of configurations of the 20V model with DWBC3 boundary conditions on an $n \times n$ square grid is the same as the number of domino tilings of the triangle $\mathcal{T}_{n}$.

We have checked this conjecture numerically up to size $n=6$.

\subsection{Pentagonal extensions of the DWBC3 conjecture}

\subsubsection{The 20V-DWBC3 model on a pentagon}

We now consider a variant of the model 20V-DWBC3 on a "pentagon" $P_{n, k}$ of the original triangular lattice. Starting from a rectangular grid of shape $(n+k) \times n$, we impose that the $n$ top external horizontal edges along the West boundary be occupied by paths, while the $k$ bottom ones be empty (vacancies), and impose the same condition as for DWBC3 on vertical external edges along the South boundary, while all other external edges are unoccupied (see Fig. 18 for an illustration with $n=6$ and $k=3$ ). Due to the nonintersection constraint which freezes some portions of the paths, the effective domain reduces to a pentagon of $\operatorname{shape}^{7}(n-1) \times k \times(n-k-1) \times(n+k-1) \times(n-1)$ (see

\footnotetext{
${ }^{7}$ Note the distinction between the notion of grid and that of shape: the size of a shape is measured in actual length of its sides, which is one less than the measure on a grid.
} 
Fig. 18). This holds for $k<n-1$. For $k \geqslant n-1$, the effective domain degenerates into a tetragon of shape $(n-1) \times(n-1) \times(2 n-2) \times(n-1)$ independent of $k$, which can be viewed as half of a regular hexagon on the original triangular lattice.

The number $p_{n, k}$ of osculating Scröder path configurations on $P_{n, k}$ is easily computed by transfer matrix techniques. The first few are listed below for $n=1,2, \ldots, 6$

$$
\begin{aligned}
& p_{n, 0}=1,3,29,901,89893,28793575 \ldots \\
& p_{n, 1}=1,4,56,2640,411840,210613312 \ldots \\
& p_{n, 2}=1,4,60,3268,628420,417062340 \ldots \\
& p_{n, 3}=1,4,60,3328,675584,495222784 \ldots \\
& p_{n, 4}=1,4,60,3328,678912,507356160 \ldots \\
& p_{n, 5}=1,4,60,3328,678912,508035072 \ldots
\end{aligned}
$$

As expected, we note a saturation property of $p_{n, k}$ which becomes independent of $k$ for $k \geqslant n-1$.

\subsubsection{Tilings of extended triangles}

We start from the triangular domain $\mathcal{T}_{n}$ of Sect. 6.2.2 above, and consider the following extensions $\mathcal{T}_{n, k}, k=0,1, \ldots, n$. Focussing on the non-intersecting Schröder path description of the tiling configurations given by the example (c) above, $\mathcal{T}_{n, k}$ corresponds to raising by $k$ vertical steps the top border of the domain accessible to the paths, while keeping identical starting and endpoints. In practice, for $k \leqslant n-1$, the new effective domain $\mathcal{T}_{n, k}$ accessible to the Schröder paths takes the following shape (represented here for $n=4$ ) which can be viewed as Aztec-like extensions of $\mathcal{T}_{n}$ :

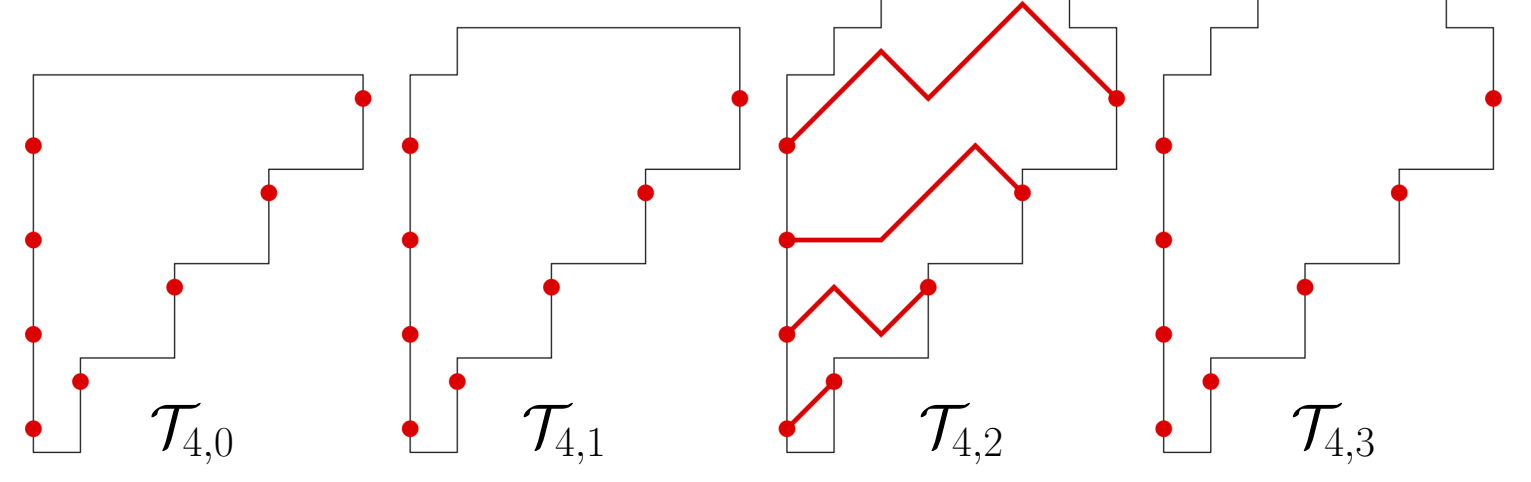

As a result, the number of configurations augments, until it reaches a threshold at $k=$ $n-1$, since, for $k \geqslant n-1$, raising the top border further no longer affects the number of configurations as the paths never reach this height.

The counting of tiling configurations is readily performed by use of the LGV formula for the corresponding Schröder paths:

$$
T\left(\mathcal{T}_{n, k}\right)=\operatorname{det}_{0 \leqslant i, j \leqslant n-1}\left(S_{2 i, 2 j+1}^{(2 n-1+k)}(2 j+1)\right)
$$



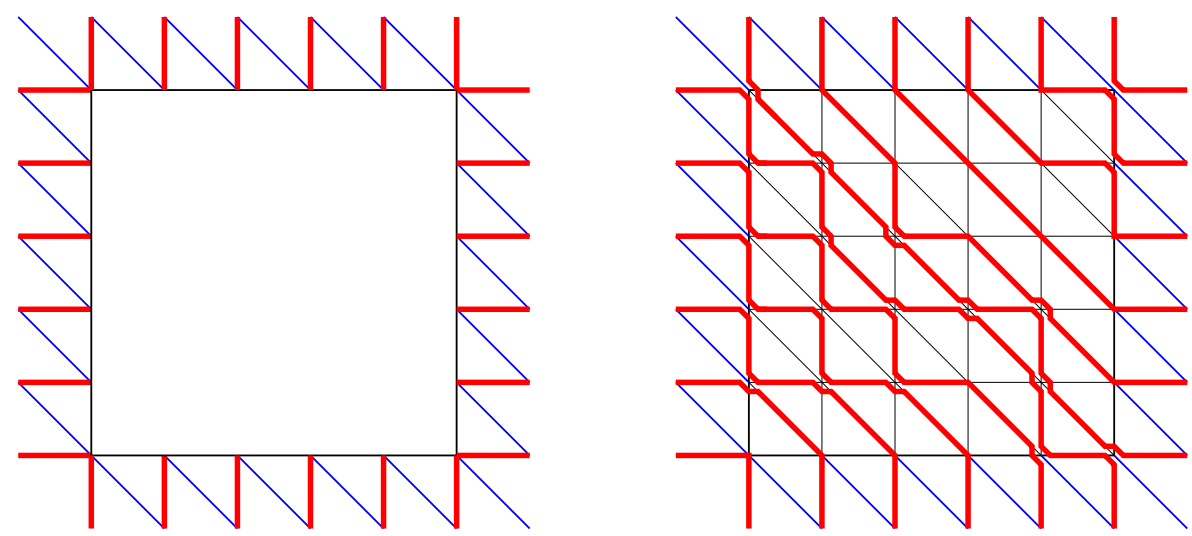

Figure 19: Left: 20V-DWBC4 on an $n \times n$ square grid: all horizontal and vertical external edges are occupied by paths, while diagonals are empty. Right: A sample osculating Schröder path configuration.

where we have simply raised the top boundary by $k$. As a result, we find perfect agreement between the numbers $p_{n, k}$ and $T\left(\mathcal{T}_{n, k}\right)$.

\subsubsection{The extended DWBC3 conjecture}

The remarkable coincidence between the numbers in Sections 6.3.1 and 6.3.2 leads to the following:

Conjecture 11. We conjecture that the number of configurations of the $20 \mathrm{~V}$ model with extended DWBC3 boundary conditions on a pentagon $P_{n, k}$ is equal to the number of domino tilings of the extended triangle $\mathcal{T}_{n, k}$ for all $n, k$.

The conjecture has been checked numerically for $n$ up to 6 and arbitrary $k$.

\subsection{More Domain Wall Boundary Conditions with no conjecture}

We have considered some other variants of the DWBC boundary conditions, but found no conjecture for those. First we studied the 20V with the DWBC4 illustrated in Fig. 19 for the osculating Schröder path formulation, in which all horizontal and vertical external edges of a square $n \times n$ grid are occupied by paths, all the other external edges being unoccupied $^{8}$. The transfer matrix calculation leads to the following sequence:

$$
1,3,59,7813,6953685,41634316343 \ldots
$$

for which we have found no other interpretation.

\footnotetext{
${ }^{8}$ We use here the denomination DWBC4 for simplicity although the boundary conditions do not infer the creation of domain walls in general, as exemplified by the trivial configuration where all horizontal (resp. vertical, diagonal) arrows point East (resp. South, Northwest).
} 


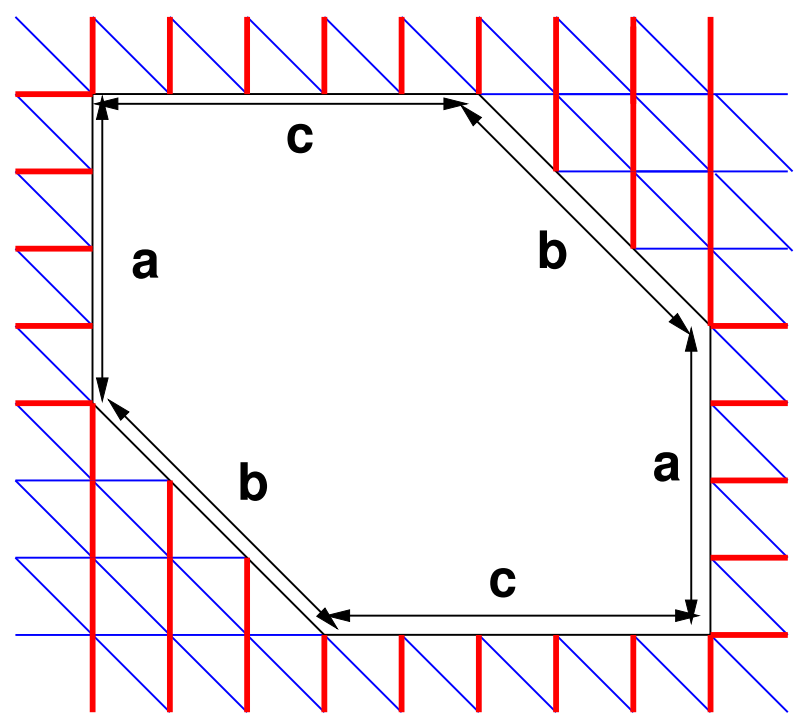

Figure 20: The hexagonal extension of 20V-DWBC4 obtained on a rectangular $(a+b+1) \times$ $(b+c+1)$ grid by imposing $b$ vacancies on the bottom West and top East boundaries.

\begin{tabular}{|l|l|l|l|l|l|l|l|}
\hline $\mathrm{b}, \mathrm{c}$ & $\mathrm{a}=\mathbf{0}$ & $\mathbf{1}$ & $\mathbf{2}$ & $\mathbf{3}$ & $\mathbf{4}$ & $\mathbf{5}$ & $\mathbf{6}$ \\
\hline $\mathbf{0 , 1}$ & 1 & 3 & 8 & 21 & 55 & 144 & 377 \\
\hline $\mathbf{0 , 2}$ & 1 & 8 & 59 & 415 & 2874 & 19810 & 136358 \\
$\mathbf{1 , 1}$ & 3 & 11 & 41 & 153 & 571 & 2131 & 7953 \\
\hline $\mathbf{0 , 3}$ & 1 & 21 & 415 & 7813 & 143336 & 2598735 & 46881130 \\
$\mathbf{1 , 2}$ & 8 & 85 & 959 & 10934 & 124869 & 1426389 & 16294360 \\
$\mathbf{2 , 1}$ & 5 & 23 & 103 & 456 & 2009 & 8833 & 38803 \\
\hline $\mathbf{0 , 4}$ & 1 & 55 & 2874 & 143336 & 6953685 & 331859360 & 15697347566 \\
$\mathbf{1 , 3}$ & 21 & 604 & 19018 & 615405 & 20055060 & 654666505 & 21378877706 \\
$\mathbf{2 , 2}$ & 20 & 333 & 5331 & 83821 & 1305844 & 20250090 & 313317426 \\
$\mathbf{3 , 1}$ & 7 & 39 & 201 & 1000 & 4888 & 23673 & 114087 \\
\hline $\mathbf{0 , 5}$ & 1 & 144 & 19810 & 2598735 & 331859360 & 41634316343 & 5164420164680 \\
$\mathbf{1 , 4}$ & 55 & 4194 & 355234 & 31391724 & 2816672309 & 254000932538 & 22940968768675 \\
$\mathbf{2 , 3}$ & 76 & 4151 & 213173 & 10696445 & 530068706 & 26081095911 & 1278122145554 \\
$\mathbf{3 , 2}$ & 36 & 881 & 18859 & 379449 & 7391755 & 141473217 & 2681264915 \\
$\mathbf{4 , 1}$ & 9 & 59 & 343 & 1880 & 9976 & 51944 & 267385 \\
\hline
\end{tabular}

Table 1: The first numbers $N_{a, b, c}$ for $a \in[0,6], b \geqslant 0, c>0$ and $b+c \leqslant 5$. 

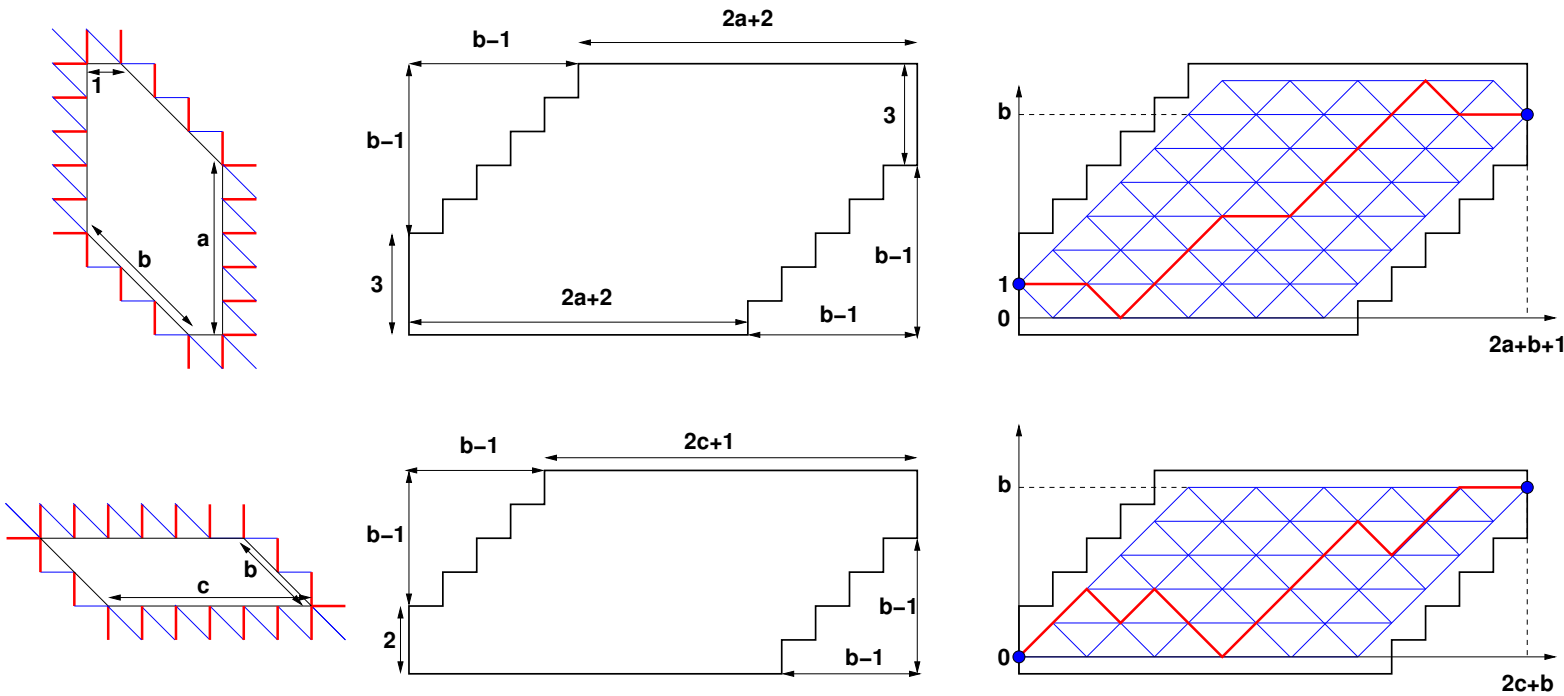

Figure 21: Top: the number of 20V-DWBC4 configurations on the hexagon $(a, b, c=1)$ (left) is conjecturally identified with the number of domino tilings of a domain (center), in bijection with configurations of a single Schröder path (right) from $(0,1)$ to $(2 a+b+1, b)$, constrained to stay on a strip $0 \leqslant y \leqslant b+1$. Bottom: the number of 20V-DWBC4 configurations on the hexagon $(a=0, b, c)$ (left) is conjecturally identified with the number of domino tilings of a domain (center), in bijection with configurations of a single Schröder path (right) from $(0,0)$ to $(2 c+b, b)$, constrained to stay on a strip $0 \leqslant y \leqslant b$.

An easy generalization consists in considering arbitrary rectangular grids of size $(a+$ $b+1) \times(b+c+1)$, and imposing that all vertical external edges be occupied while only the top $a+1$ horizontal ones on the West boundary, and bottom $a+1$ horizontal ones on the East boundary be occupied (see Fig. 20). Equivalently the bottom $b$ horizontal external edges on the West boundary and the top $b$ ones on the East boundary are empty. This leads to numbers $N_{a, b, c}$ of configurations.

The case $b=0$ corresponds to rectangular grids of arbitrary size $(a+1) \times(c+1)$, for which the boundary condition DWBC4 still makes sense. In particular, $N(n-1,0, n-1)$ reproduces the above sequence (6.3). Note also that $N_{a, b, 0}=1$, as there is a unique, fully osculating, configuration in that case, as illustrated for $(a, b, c)=(2,3,0)$ below:

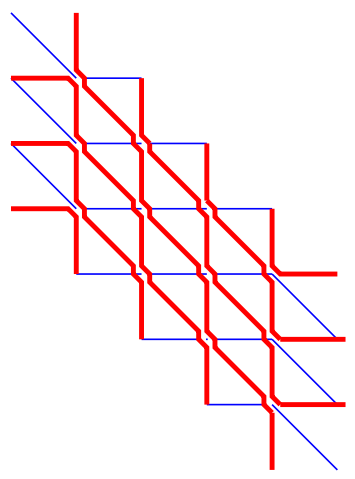


We have listed some of the non-trivial numbers $N_{a, b, c}$ in Table 6.4. So far we have identified conjecturally only the numbers $N_{a, b, 1}$ and $N_{0, b, c}$ as also counting the domino tilings of plane domains depicted in Fig. 21. The latter are easily enumerated by the configurations of a single Schröder path with fixed ends, and constrained to remain within a strip, as shown on the right. More precisely, we have, with the notation of Section 6.2.2:

\section{Conjecture 12 .}

$$
N_{a, b, 1}=S_{1, b}^{(b+1)}(2 a+b+1), \quad N_{0, b, c}=S_{0, b}^{(b)}(2 c+b) .
$$

\section{Alternating Phase Matrices}

We may reformulate the $20 \mathrm{~V}$ models with DWBC1,2,3 in terms of Alternating Phase Matrices (APM), which generalize the Alternating Sign Matrices (ASM), with entries among 0 and the sixth roots of unity, and with specific alternating conditions.

\subsection{From 20V configurations to APM}

In the case of the $6 \mathrm{~V}$ model with DWBC, one possible construction of ASM is by viewing the six possible vertex configurations as "transmitters" or "reflectors" of the orientation of the arrows when going say from left to right and from top to bottom. A vertex either reflects or transmits both directions as a consequence of the ice rule. Starting from a $6 \mathrm{~V}-\mathrm{DWBC}$ configuration, we map vertices to entries of the ASM built according to the following rules:

1. If the vertex is a transmitter, the entry of the ASM is 0 ;

2. If the vertex is a reflector, the entry is +1 if the horizontal arrows point inwards, and -1 otherwise.

In the case of the $20 \mathrm{~V}$ model, each vertex is now viewed as a triple of reflectors or transmitters along the horizontal, vertical and diagonal directions, say going from NW to SE. To each vertex of a 20V-DWBC1,2 or 3 configuration, we may assign a triple $(h, v, d)$ of elements of $\{0,1,-1\}$ where $h, v$ and $d$ indicate the transmitter of reflector state of the horizontal, vertical and diagonal directions respectively, with the following rules:

1. If the vertex is a transmitter along a direction, the corresponding entry of the triple is 0 ;

2. If the vertex is a reflector along a direction, the corresponding entry is +1 if the arrows point inwards, and -1 otherwise.

For each triple at a vertex, we have the condition $h+d+v=0$ as a consequence of the $i c e$ rule, which imposes that either the vertex is a transmitter in all three directions (and then $h=d=v=0$ ), or it is a transmitter in only one direction and a reflector in the other two, and in this latter case, one reflected pair points inwards and the other outwards, 
so that the corresponding entries of the triple have zero sum. This gives rise to seven possible triples: the triple $(0,0,0)$ encountered for 8 of the 20 vertices and six non-zero possible triples, according to the dictionary below in terms of osculating Schröder paths:
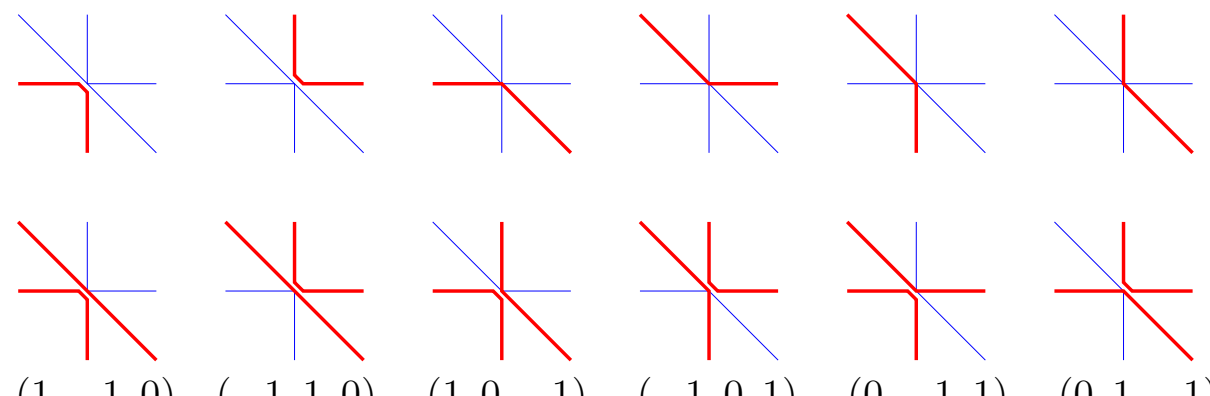

$$
(1,-1,0) \quad(-1,1,0)
$$

1

$$
(1,0,-1)
$$$$
-\omega
$$

$(-1,0,1)$

$\omega$
$(0,-1,1)$
$-\omega^{2}$

We have also indicated an alternative bijective coding using the sixth roots of unity with $\omega=e^{2 i \pi / 3}$, the weight of a triple $(h, v, d)$ being bijectively mapped onto the complex number $-\omega h+\omega^{2} v$ (which includes the coding $\left.(0,0,0) \mapsto 0\right)$. This allows to assign to each $20 \mathrm{~V}$ configuration of size $n$ with DWBC1,2 or 3 an $n \times n$ matrix with elements either 0 or in the set of sixth roots of unity: we shall call such matrices Alternating Phase Matrices (APM) of type 1,2 or 3 respectively. Note that all the ASMs are realized as APMs (with only \pm 1 non-zero entries), in all three types. To see why, start from the osculating path formulation of ASM on the square grid, and then superimpose diagonal entirely empty or entirely occupied lines, according to the diagonal external edge states pertaining to the chosen DWBC. This generates APMs with entries 0, 1 and -1 only (corresponding to the first two columns in the figure above) equal to those of the corresponding ASMs.

Alternatively, we may understand the sixth root of unity weights as a weighting of the turns taken by individual paths, with the rule that the total weight at a vertex is the sum of the turning weights of all the paths visiting it. More precisely, the expression $-\omega h+\omega^{2} v$ for vertex weights may be understood as the sum over all two-step paths $p=\left(p_{\text {in }}, p_{\text {out }}\right)$ visiting the vertex of turning weights equal to the variation $\operatorname{turn}(p):=\eta\left(p_{\text {out }}\right)-\eta\left(p_{\text {in }}\right)$ of an edge variable $\eta$ with the following dictionary:

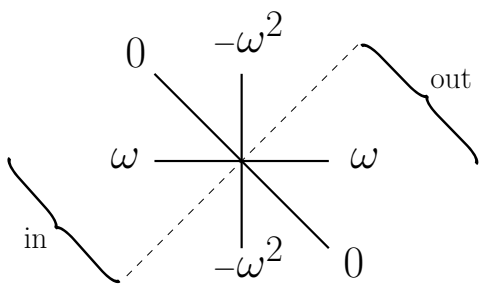

where we have indicated the value of $\eta$ for each in/out edge. With this dictionary, a path which does not turn (transmitter direction) receives the turning weight 0 while we have the following assignment of turning weight for respectively horizontal, diagonal and vertical incoming paths (we have indicated the direction of travel of each path by a straight 
arrow):

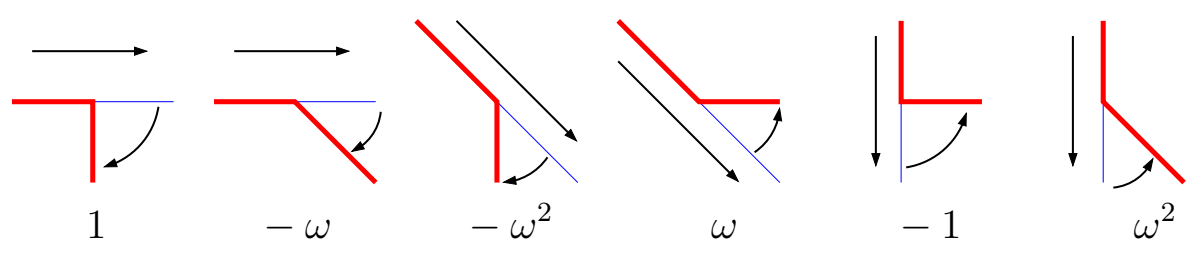

It is a straightforward exercise to check that all the weights of (7.2) are indeed the sums of turning weights of all their two-step paths (this is indeed guaranteed by the mapping $\left.(h, v, d) \mapsto-\omega h+\omega^{2} v\right)$. For instance, the fifth weight from left on the bottom line is $1+\omega=-\omega^{2}$, namely equal to the sum of the first and fourth weights on (7.3). Moreover, a useful consequence of the definition is that the turning weights of a given path $p=$ $\left(p_{0}, p_{1}, \ldots, p_{k}\right)$ add up along the path into a telescopic sum equal to

$$
\operatorname{turn}(p):=\sum_{\ell=1}^{k} \operatorname{turn}\left(\left(p_{\ell-1}, p_{\ell}\right)\right)=\eta\left(p_{k}\right)-\eta\left(p_{0}\right)=\operatorname{turn}\left(\left(p_{0}, p_{k}\right)\right)
$$

depending only on the orientations of its first and last edges.

\subsection{APM of type 1, 2 and 3: definitions and properties}

The matrix triple entries $(h, v, d)$ coming from $20 \mathrm{~V}$ configurations are further constrained by reflection/transmission properties along the horizontal, vertical and diagonal directions. In all three cases of DWBC1,2,3 all the external horizontal arrows point towards the grid and all the external vertical arrows point away from it. If we follow any horizontal line from left to right, the first arrow on the West side points to the right, and must be reflected at least once as it points left when it exits on the East side. It must in fact be reflected an odd number of times. The corresponding entries $h_{j}$ of the triples associated to the vertices $j=1,2, \ldots, n$ visited from left to right must therefore alternate between $1,-1,1, . ., 1$ whenever they are non-zero. The same reasoning for the vertical directions leads, from top to bottom, to an alternation of the entries $v_{i}$ in each column between $-1,1,-1, \ldots,-1$ whenever non-zero.

Finally a last alternance condition holds along the diagonal directions, but is different for DWBC1,2 and 3. For DWBC3, as all external diagonal edges are empty, the entries $d_{i}$ along each diagonal visited from top to bottom, must alternate between $-1,1,-1, \ldots, 1$ when they are non-zero: note that there is indeed always an even, possibly zero number of reflectors as the arrows at both ends point towards Northwest. For DWBC1, recall that the external diagonal edges are occupied in the lower triangular part and empty in the strictly upper triangular part of the $n \times n$ grid. As a consequence, the entries $d_{i}$ (labeled by, say the row index $i$ in increasing order) along each diagonal in the strictly upper triangular part obey the same rule as in DWBC3, namely alternate between $-1,1,-1, \ldots, 1$ when they are non-zero, but the entries $d_{i}$ along each diagonal in the lower triangular part obey the opposite rule, namely alternate between $1,-1,1, \ldots,-1$ when they are nonzero. Finally, for DWBC2 the entries $d_{i}$ along each diagonal in the upper triangular part 
alternate between $-1,1,-1, \ldots, 1$ when they are non-zero, and the entries $d_{i}$ along each diagonal in the strictly lower triangular part obey the opposite rule, namely alternate between $1,-1,1, \ldots,-1$ when they are non-zero.

These rules determine entirely the sets of APM of type 1,2,3 whose definition is summarized below.

Definition 13. We define the sets of $n \times n$ Alternating Phase Matrices of types $1,2,3$ as $n \times n$ arrays of triples of the form $\left(h_{i, j}, v_{i, j}, d_{i, j}\right)$ for $1 \leqslant i, j \leqslant n$, where $h_{i, j}, v_{i, j}, d_{i, j} \in$ $\{0,1,-1\}$ satisfy $h_{i, j}+v_{i, j}+d_{i, j}=0$ and are moreover subject to the following conditions for all types:

1. There is at least one non-zero variable $h_{i, j}$ in each row $i=1,2, \ldots, n$, and at least one non-zero variable $v_{i, j}$ in each column $j=1,2, \ldots, n$,

2. Along each row $i=1,2, \ldots, n$, the non-zero variables $h_{i, j}$ must alternate between $1,-1,1, \ldots, 1$ when $j$ ranges from 1 to $n$,

3. Along each column $j=1,2, \ldots, n$, the non-zero variables $v_{i, j}$ must alternate between $-1,1,-1, \ldots,-1$ when $i$ ranges from 1 to $n$,

and to three different conditions (4.1), (4.2), (4.3) corresponding to each type 1,2,3:

(4.1) Along each diagonal ${ }^{9} \ell \in[1-n, n-1]$, the non-zero variables $d_{i, i+\ell}$ must alternate between $-1,1,-1, \ldots, 1$ if $\ell>0$ and $1,-1,1, \ldots,-1$ if $\ell \leqslant 0$ when $i$ ranges from $\operatorname{Max}(1,1-\ell)$ to $\operatorname{Min}(n, n-\ell)$.

(4.2) Along each diagonal $\ell \in[1-n, n-1]$, the non-zero variables $d_{i, i+\ell}$ must alternate between $-1,1,-1, \ldots, 1$ if $\ell \geqslant 0$ and $1,-1,1, \ldots,-1$ if $\ell<0$ when $i$ ranges from $\operatorname{Max}(1,1-\ell)$ to $\operatorname{Min}(n, n-\ell)$.

(4.3) Along each diagonal $\ell \in[1-n, n-1]$, the non-zero variables $d_{i, i+\ell}$ must alternate between $-1,1,-1, \ldots, 1$ when $i$ ranges from $\operatorname{Max}(1,1-\ell)$ to $\operatorname{Min}(n, n-\ell)$.

APM entries are expressible ubiquitously in terms of either the above defining triples, or equivalently zero or sixth roots of unity according to the dictionaries (7.1-7.2).

Proposition 14. The $n \times n$ APM of types 1,2,3 are in bijection with respectively the $20 \mathrm{~V}-D W B C 1,2,3$ on an $n \times n$ grid.

Example 15. As a illustration the $5 \times 5$ APM of types 1,2 and 3 corresponding to the

\footnotetext{
${ }^{9}$ Here we label the diagonal from $1-n$ to $n-1$ from top to bottom, as opposed to the spectral parameter labelling $t_{k}, k=1,2, \ldots, 2 n-1$ from bottom to top. The correspondence is $k=n-\ell$.
} 
configurations depicted respectively in Figs. 1, 2 and 17 read:

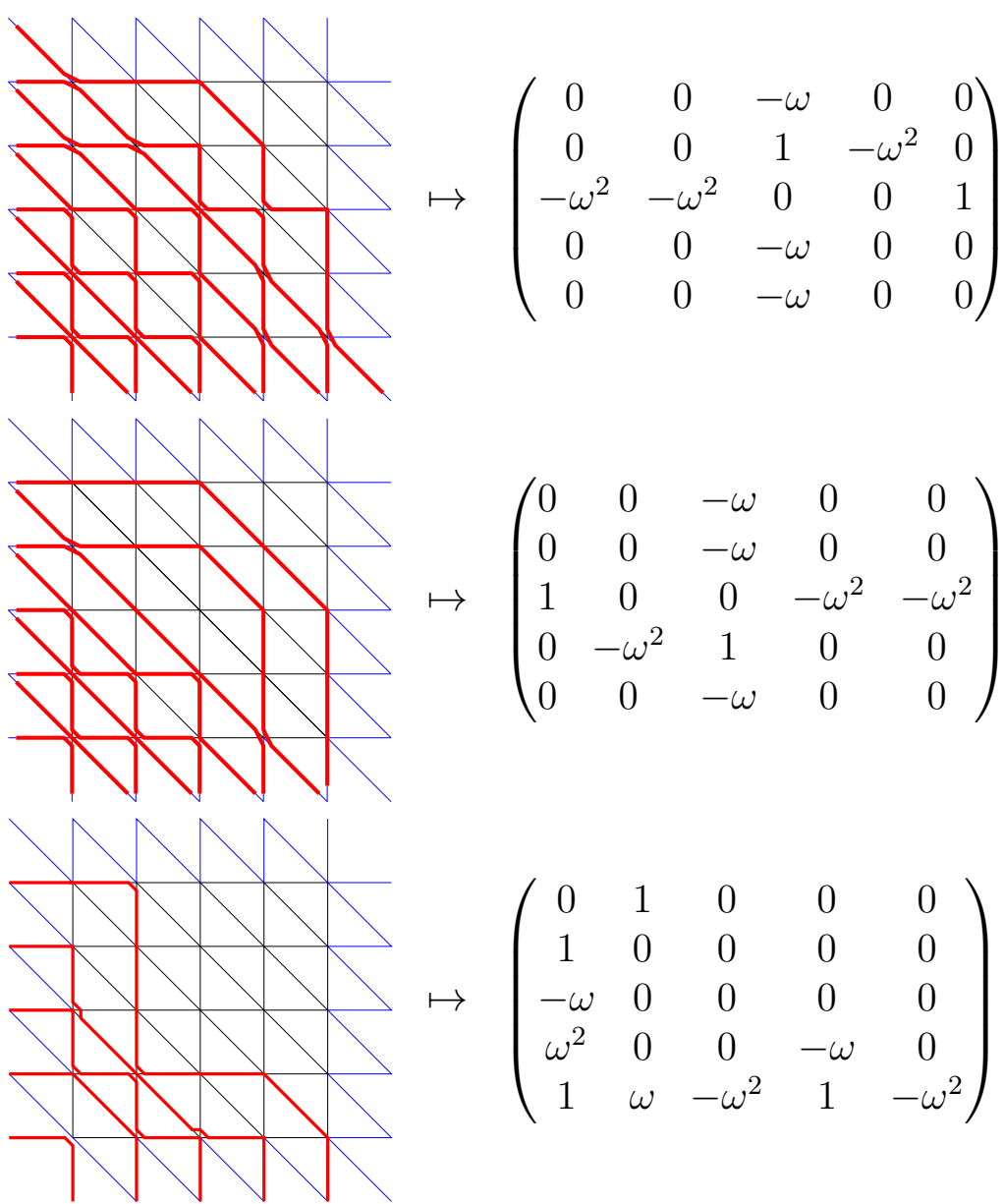

We note that the first and second APMs of respective types 1 and 2 are exchanged under a rotation by $180^{\circ}$, which matches the fact that the corresponding $20 \mathrm{~V}$ configurations are also interchanged under the same transformation.

We conclude with a simple property satisfied by all APMs introduced so far.

Proposition 16. APMs $A=\left(a_{i, j}\right)_{1 \leqslant i, j \leqslant n}$ of any type 1, 2 or 3, when expressed in terms of sixth roots of unity, have the following property:

$$
\sum_{i, j=1}^{n} a_{i, j}=n .
$$

Proof. We use the interpretation of weights in terms of turning weights for the paths (7.3). For each path $p$ in the osculating Schröder path configuration, recall the quantity $\operatorname{turn}(p)(7.4)$ defined as the sum over its turning weights. Then it is clear that the desired quantity is $\sum_{i, j=1}^{n} a_{i, j}=\sum_{p} \operatorname{turn}(p)$, where the sum extends over all the paths in the configuration. Next, we note that irrespectively of the type of DWBC, all paths have the following property: 
1. each path starting with a horizontal external edge on the West boundary ends with a vertical edge on the South boundary (call these HV paths). From (7.4), these paths have a turning weight $\operatorname{turn}(p)=-\omega^{2}-\omega=1$.

2. each path starting with a diagonal external edge on the West boundary ends with a diagonal edge on the South boundary. From (7.4), these paths have a turning weight $\operatorname{turn}(p)=0-0=0$.

The Proposition follows from the fact that there are always exactly $n \mathrm{HV}$ paths in all configurations.

\subsection{Other APMs}

Using the same dictionaries as in the previous sections, we now define APM of type 4 corresponding to 20V-DWBC4 of Section 6.4.

Definition 17. We define APM of type 4 as $n \times n$ arrays of triples of the form $\left(h_{i, j}, v_{i, j}, d_{i, j}\right)$ for $1 \leqslant i, j \leqslant n$, where $h_{i, j}, v_{i, j}, d_{i, j} \in\{0,1,-1\}$ and $h_{i, j}+v_{i, j}+d_{i, j}=0$, moreover subject to the following conditions:

1. Along each row $i=1,2, \ldots, n$, the non-zero variables $h_{i, j}$ must alternate between $1,-1,1, \ldots,-1$ when $j$ ranges from 1 to $n$,

2. Along each column $j=1,2, \ldots, n$, the non-zero variables $v_{i, j}$ must alternate between $1,-1,1, \ldots,-1$ when $i$ ranges from 1 to $n$,

3. Along each diagonal $\ell \in[1-n, n-1]$, the non-zero variables $d_{i, j}$ must alternate between $-1,1,-1, \ldots, 1$ when $i$ ranges from $\operatorname{Max}(1,1-\ell)$ to $\operatorname{Min}(n, n-\ell)$.

Proposition 18. The $n \times n$ APM of type 4 are in bijection with the configurations of the $20 \mathrm{~V}-\mathrm{DWBC} 4$ model on an $n \times n$ grid.

In particular, the zero matrix is an APM of type 4, which corresponds to all vertices being transmitters. The APM of type 4 are indeed very different from those of types 1,2,3: we note in particular that ASM do not form a subset of APM of type 4, as the DWBC4 is incompatible with the DWBC of the underlying square lattice leading to $\mathrm{ASM}^{10}$. Moreover, Proposition 16 no longer holds, and is replaced by

Proposition 19. For Any APM $A=\left(a_{i, j}\right)_{1 \leqslant i, j \leqslant n}$ of type 4, when expressed in terms of sixth roots of unity, we have the following property:

$$
\sum_{i, j=1}^{n} a_{i, j}=0
$$

\footnotetext{
${ }^{10}$ This echoes the previous remark that DWBC4 are not really domain wall inducing.
} 
Proof. We use the same argument as in the proof of Proposition 16. All $n$ paths entering from the top must exit on the right. Each of them has a turning weight $\omega-\left(-\omega^{2}\right)=-1$. All $n$ paths entering from the left must exit on the bottom. Each of them has turning weight $-\omega^{2}-\omega=+1$. The total turning weight is therefore $n-n=0$.

We also have the following refined sum rules:

Proposition 20. For Any APM $A=\left(a_{i, j}\right)_{1 \leqslant i, j \leqslant n}$ of type 4, when expressed in terms of sixth roots of unity, we have the following properties:

$$
\sum_{j=1}^{n} a_{i, j} \in \omega^{2} \mathbb{Z}, \quad \sum_{i=1}^{n} a_{i, j} \in \omega \mathbb{Z}, \quad \sum_{i=\operatorname{Max}(1,1-\ell)}^{\operatorname{Min}(n, n-\ell)} a_{i, i+\ell} \in \mathbb{Z} .
$$

Proof. We use the fomulation of the matrix entries $a_{i, j}$ as the sum of turning weights of all paths visiting the vertex $i, j$. Focussing on a given row $i$ of the matrix, the horizontal occupied edges form a union of segments of edges belonging each to a different path: $\left[a_{0}, a_{1}\right],\left[a_{2}, a_{3}\right], \ldots,\left[a_{2 k-2}, a_{2 k-1}\right]$ with $k>1, a_{0}=0 \leqslant a_{1} \leqslant \cdots \leqslant a_{2 k-2} \leqslant a_{2 k-1}=n+1$. Inside each segment all vertices are transmitters, with turning weights 0 . All the junctures $a_{1}, a_{2}, \ldots, a_{2 k-2}$ are turning points. Inspecting the possibilities of local configurations of paths through these points, there are two possibilities of exiting the row at each $a_{j}$ for odd $j<2 k-1$ :

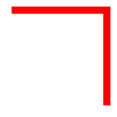

1

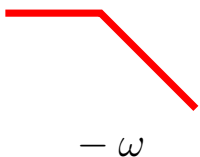

and two possibilities to enter the row at each $a_{j}$ for even $j>0$ :
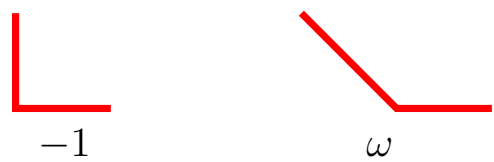

Let $\theta_{j}$ denote the corresponding turning weight, with $\theta_{j} \in\{1,-\omega\}$ for odd $j$ and $\theta_{j} \in$ $\{-1, \omega\}$ for even $j$. Summing over all turning weights along the row $i$ gives $\sum_{j=1}^{n} a_{i, j}=$ $\sum_{j=1}^{k-1}\left(\theta_{2 j-1}+\theta_{2 j}\right)$ : this includes double and also triple osculations, as the latter must have a transmitter diagonal which does not affect the turning weight. The quantity $\theta_{2 j-1}+\theta_{2 j}$ may take only the values: $1-1=0,1+\omega=-\omega^{2},-\omega-1=\omega^{2}$ and $-\omega+\omega=0$, all in $\omega^{2} \mathbb{Z}$, and the first assertion of the Proposition follows. The second and third ones follow from a similar argument.

Example 21. As a illustration, the $6 \times 6$ APM of type 4 corresponding to the configuration 
on the left in Fig. 19 reads:

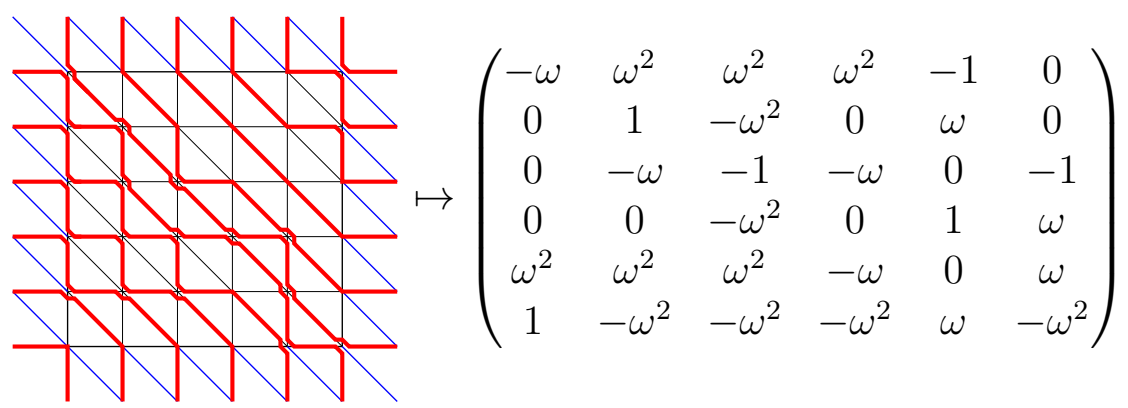

The sum rules of Props.19 and 20 are easily checked. We find row sums: $4 \omega^{2},-2 \omega^{2}, 2 \omega^{2}$, $-2 \omega^{2}, 3 \omega^{2},-5 \omega^{2}$, column sums: $-2 \omega,-2 \omega, \omega,-2 \omega, 2 \omega, 3 \omega$, and diagonal sums: $1,0,0$, $0,1,1,1,-1,-2,-1,0$, each adding up to 0 .

\section{Discussion/Conclusion}

In this paper we have considered the two-dimensional ice model of statistical physics on the triangular lattice, the $20 \mathrm{~V}$ model, from a combinatorial point of view. In particular, we have defined analogs of the known DWBC for the $6 \mathrm{~V}$ model, and investigated their possible combinatorial content, using as much as possible the underlying integrable structure of the models.

\subsection{DWBC1,2: summary and perspectives}

The first class of boundary conditions DWBC1,2 have displayed a remarkably rich combinatorial content. We have shown in particular that the configurations of the models are equinumerous to the domino tilings of a quasi-square Aztec-like domain with a central cross-shaped hole and with quarter-turn symmetry. We also presented a refined version, in the same spirit as the Mills-Robins-Rumsey conjecture [MRR86] for DPP, fully proved in [BDFZJ12]. Note that this coincidence of (refined) partition functions is still lacking a direct bijective interpretation. However, such a canonical bijection is not known even for the ASM-DPP correspondence.

The $20 \mathrm{~V}$ model presents interesting new features compared to the $6 \mathrm{~V}$ model. Its osculating path version involves Schröder paths with three different kinds of steps (horizontal, vertical, diagonal). Note that the same paths, but with a stronger non-intersecting condition are involved in the description of domino tilings of the holey square. It is easy to keep track say of the diagonal steps when dealing with a single path (with a weight $\gamma$ per diagonal step), as well as when dealing with families of such non-intersecting paths (see Ref. [DFG19] for the problem of tiling Aztec rectangles with defects). The corresponding decoration is easy to implement in the holey square tiling problem (where $\gamma$ is simply a weight for one kind of domino). Unfortunately, it is easily checked that the partition functions of the 20V-DWBC1,2 and that of domino tilings of the holey square no longer 
match for $\gamma \neq 1$. Besides, it turns out that weights incorporating a factor $\gamma$ per diagonal step of path in the $20 \mathrm{~V}$ model are non-longer integrable in general, namely cannot be obtained by special choices of spectral parameters. On the other hand, it would be interesting to find a weighting of the $20 \mathrm{~V}$ model equivalent to the $\gamma$-deformation of the tiling.

Another easily implementable natural weight in the particular context of the quarterturn symmetric domino tilings of the holey Aztec square is a weight $\theta$ per path in the formulation as non-intersecting paths on a cone of Section 4. The suitably modified partition function reads $\operatorname{det}\left(\mathbb{I}_{n}+\theta M_{n}\right)$ in the notations of Theorem 4: when evaluating the determinant via the Cauchy-Binet formula, we indeed pick a factor $\theta$ per row of the chosen submatrix of $M_{n}$. It would also be interesting to find a weighting of the $20 \mathrm{~V}$ model that corresponds to this.

Finally, like in the DPP case [BDFZJ13], further refinements could in principle be derived, by use of the Desnanot-Jacobi relation. We hope to return to these questions in future work.

\subsection{The DWBC3 conjecture}

Proving the DWBC3 conjecture 10 is the next challenge. Let us mention that the partition function $Z^{20 V_{B C 3}}(n)$ of the 20V-DWBC3 model on an $n \times n$ grid, due to the integrable nature of its weights, can be related to a partition function of the $6 \mathrm{~V}$ model on a $2 n \times 2 n$ grid of square lattice, by unraveling the diagonal lines in a way similar to that of Section 3.1 .

More precisely, we start from the general DWBC3 partition function, with arbitrary Kagome spectral parameters $z_{i}, w_{j}, i, j=1,2, \ldots, n$ and homogeneous $t_{k}=t$, $k=1,2, \ldots, 2 n-1$. First, we note that the $(n-1)$ bottom diagonal lines are "imprisoned" due to the alternating orientations of external arrows on the West and South boundaries, and the corresponding lines cannot be expelled like in the case of Section 3.1. The top $n$ on the other hand could in principle be disposed of in the same way as before. However, it proves more interesting to keep them and to deform the lines in the manner illustrated in Fig. 22. The idea is to place the former nodes of the sublattices 2 and 3 of the Kagome on square (sub-)lattices, still denoted by 2,3, and to form artificial new nodes at the kissing between pairs of deformed diagonal lines, and positioned on a square sublattice denoted by 4 (see Fig. $22(\mathrm{~b})$ ). At these nodes, the kissing condition is guaranteed by choosing vertex weights $\left(a_{4}, b_{4}, c_{4}\right)=(1,0,1)$, where the vanishing of the weight $b_{4}$ ensures the transmission of the arrow orientation from top to right and from left to bottom for these artificially created vertices. By careful inspection of the vertex weights whenever some $t_{k}=t$ is involved, we find that the weights for sublattices 2 and 3 may be expressed (up to a global multiplicative factor $q$ for sublattice 2 and $q^{-1}$ for sublattice 3) with the same definition as that used for the sublattice 1 of the Kagome lattice (or equivalently given by (3.7)) provided we change $t \rightarrow q^{2} t$ in the definition of horizontal spectral parameters and $t \rightarrow q^{-2} t$ in the definition of the vertical ones (see Fig. 22 (c)). Remarkably, with these modified spectral parameters and this very same 

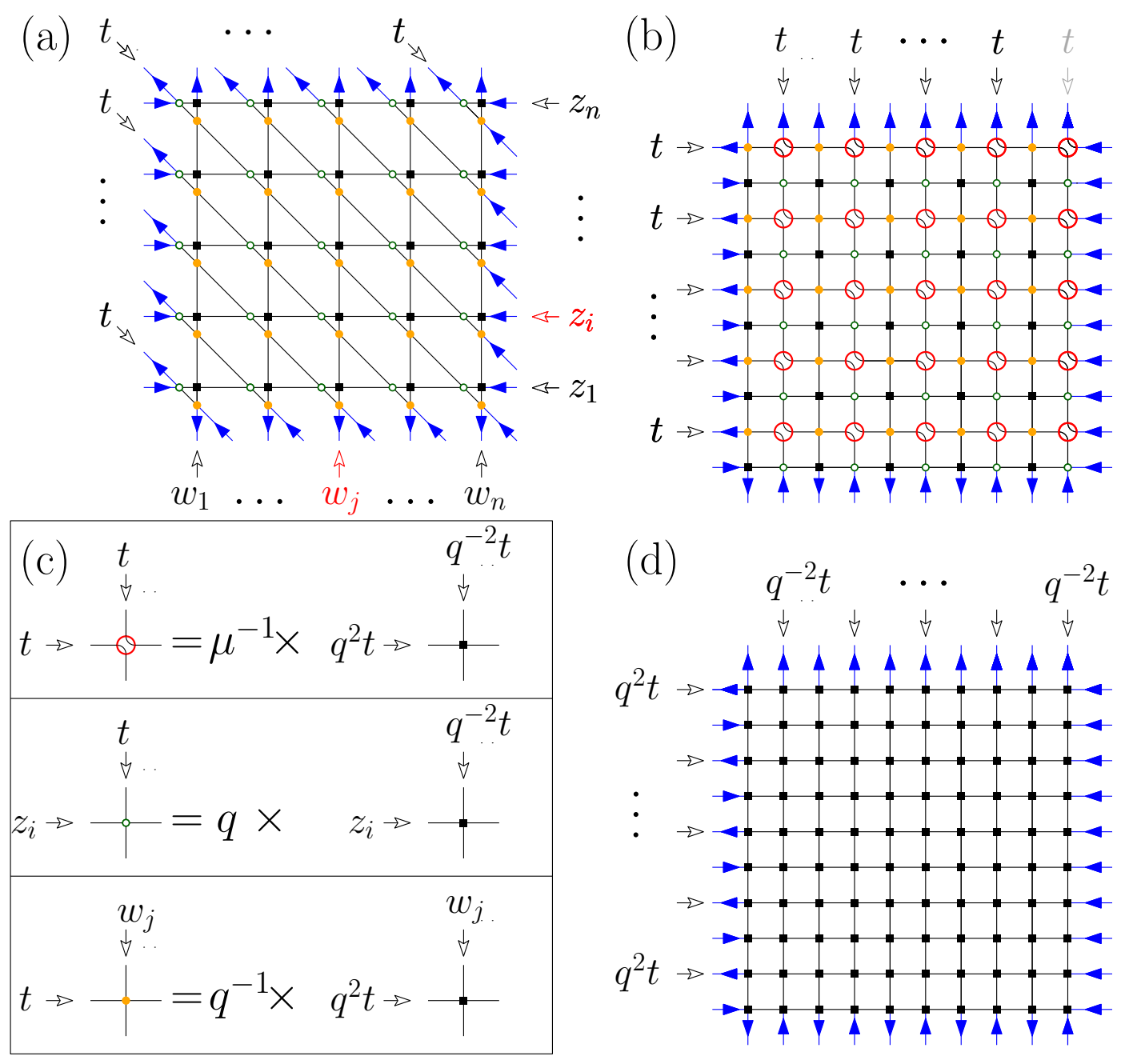

Figure 22: Starting from the 20V-DWBC3 model on an $n \times n$ grid in its equivalent Kagome lattice formulation (a), we deform the diagonal lines so as to form an intermediate square grid of size $2 n \times 2 n$ (b) with four sublattices corresponding to the 3 original sublattices 1,2,3 of the Kagome lattice indicated respectively with black squares (1), empty circles (2), filled circles (3) plus an extra sublattice 4 of kissing points (circled), including the top right kissing point with an added trivial corner oriented line. Thanks to the equivalence to $6 \mathrm{~V}$ weights indicated in (c), the model is finally reexpressed as a staggered $6 \mathrm{~V}$ model with appropriate spectral parameters $(\mathrm{d})$. 
unified definition, the $6 \mathrm{~V}$ weights on the sublattice 4 would be

$$
\left(A\left(q^{2} t, q^{-2} t\right), B\left(q^{2} t, q^{-2} t\right), C\left(q^{2} t, q^{-2} t\right)\right)=\left(q^{2}-q^{-2}\right) t \times(1,0,1)
$$

which reproduce precisely the desired weights $\left(a_{4}, b_{4}, c_{4}\right)$ up to the proportionality factor $\mu=\left(q^{2}-q^{-2}\right) t$. Otherwise stated, the weights on the four sublattices correspond (up to global factors $1, q, q^{-1}$ and $\mu^{-1}$ for sublattices $1,2,3,4$ respectively) to $6 \mathrm{~V}$ weights for a consistent set of spectral parameters on the horizontal and vertical lines of a $2 n \times 2 n$ grid.

The other spectral parameters $z_{i}$ and $w_{j}$ have remained unchanged in the process. Taking them to the homoegeous $20 \mathrm{~V}$ values $z_{i}=q^{6} t$ and $w_{j}=q^{-6} t$, we obtain a new $6 \mathrm{~V}$ model now on a $2 n \times 2 n$ grid, but with staggered boundary conditions and weights:

$$
\begin{aligned}
& \left(a_{1}, b_{1}, c_{1}\right)=\left(A\left(q^{6} t, q^{-6} t\right), B\left(q^{6} t, q^{-6} t\right), C\left(q^{6} t, q^{-6} t\right)\right)=\mu(1, \sqrt{2}, 1) \\
& \left(a_{2}, b_{2}, c_{2}\right)=q\left(A\left(q^{6} t, q^{-2} t\right), B\left(q^{6} t, q^{-2} t\right), C\left(q^{6} t, q^{-2} t\right)\right)=q^{3} \mu(\sqrt{2}, 1,1) \\
& \left(a_{3}, b_{3}, c_{3}\right)=q^{-1}\left(A\left(q^{2} t, q^{-6} t\right), B\left(q^{2} t, q^{-6} t\right), C\left(q^{2} t, q^{-6} t\right)\right)=q^{-3} \mu(\sqrt{2}, 1,1) \\
& \left(a_{4}, b_{4}, c_{4}\right)=(1,0,1)
\end{aligned}
$$

respectively on the sublattices $1,2,3$ (as in (2.7)) and 4. Recalling the choice of spectral parameter $t(2.8)$, leading to $\mu^{3}=1 / 2$, to ensure that the original $20 \mathrm{~V}$ weights are all 1 , the net result is a re-expression of the total number of configurations of the 20V-DWBC3 as:

$$
Z^{20 V_{B C 3}}(n)=\mu^{n^{2}}\left(q^{3} \mu\right)^{n^{2}}\left(q^{-3} \mu\right)^{n^{2}} Z_{W S}^{6 V_{\text {staggered }}}(2 n)=\frac{1}{2^{n^{2}}} Z_{W S}^{6 V_{\text {staggered }}}(2 n),
$$

where $Z_{W S}^{6 V_{\text {staggered }}}(2 n)$ denotes the partition function of the staggered $6 \mathrm{~V}$ model on a $2 n \times 2 n$ grid, with weights $(a, b, c)$ respectively equal to $(1, \sqrt{2}, 1),(\sqrt{2}, 1,1),(\sqrt{2}, 1,1)$ and $(1,0,1)$ on the sublattices $1,2,3,4$, and with alternating external arrow orientations on the West and South boundaries (indicated by the index $W S$ ), entering arrows on the East and outgoing arrows on the North (as in Fig. 22(d)).

In fact, the same transformation could be performed on the $20 \mathrm{~V}$ model on an $n \times n$ grid with arbitrary boundary conditions. In particular, this allows to re-express the partition function for the $20 \mathrm{~V}$-DWBC4 model as a staggered $6 \mathrm{~V}$ model with the same definition of weights as above, but with alternating external arrow orientations on all (West, South, East, North) boundaries:

$$
Z^{20 V_{B C 4}}(n)=\frac{1}{2^{n^{2}}} Z_{W S E N}^{6 V_{\text {staggered }}}(2 n) .
$$

The same transformation for the DWBC1 20V model leads to an alternative re-expression as:

$$
Z^{20 V_{B C 1}}(n)=\frac{1}{2^{n^{2}}} Z^{6 V_{\text {staggered }}}(2 n),
$$

where the staggered $6 \mathrm{~V}$ model has domain wall boundary conditions, i.e. all horizontal external arrows pointing towards the domain, and all vertical external arrows pointing away from the domain. 
There are no known general determinantal formulas for the partition functions of the staggered $6 \mathrm{~V}$ model. However, the latter can be solved by use of algebraic Bethe Ansatz, so there is some hope of transforming the relevant partition functions into some simpler objects. We also leave this investigation to some future work.

\subsection{Symmetry classes}

Among the many questions that remain, an interesting direction which is similar to that in the case of $6 \mathrm{~V} / \mathrm{ASM} /$ Rhombus Tilings correspondences is the introduction of symmetry classes, obtained by restricting to configurations with some specified symmetry.

Our problem allows for less possible symmetries than the original $6 \mathrm{~V}$ one, as we have broken the natural symmetries of the square domain in our choices of DWBC. Nevertheless, we have identified two interesting symmetry classes for the DWBC1,2 models, one for DWBC3 and two for DWBC4.

In osculating Schröder path language, the first symmetry, common to all DWBC1,2,3 and 4 , is simply the symmetry under reflection w.r.t. the first diagonal, which clearly respects all four choices of boundary conditions. In the APM language (with 0 and sixth root of unity entries), this symmetry amounts, for the APM $A$, to the condition:

$$
A_{n+1-j, n+1-i}^{*}=A_{i, j} \quad(i, j=1,2, \ldots, n),
$$

where the complex conjugation interchanges $\omega$ and $\omega^{2}$. We denote the APMs having this symmetry by Symmetric Alternating Phase Matrices (SAPM), which come in four types.

The second symmetry is more subtle and applies only to DWBC1 and 2. It is the composition of a reflection w.r.t. the second diagonal and the complementation which interchanges occupied and empty edges, while the central diagonal line remains entirely occupied (DWBC1) or entirely empty (DWBC2). Note that in the cases of DWBC3 and 4 this transformation would be incompatible with the boundary conditions. In the APM language, this amounts to the condition:

$$
A_{j, i}^{*}=A_{i, j} \quad(i, j=1,2, \ldots, n)
$$

namely that the corresponding APMs be Hermitian. We denote the APMs having this symmetry by Transpose Conjugate Alternating Phase Matrices (TCAPM).

The last symmetry occurs only for DWBC4: it is the rotation of the grid by $180^{\circ}$. In the APM language, this amounts to the condition:

$$
A_{n+1-i, n+1-j}=-A_{i, j} \quad(i, j=1,2, \ldots, n) .
$$

We denote the APM having this symmetry by Half-Turn (symmetric) Alternating Phase Matrices (HTAPM).

Using transfer matrix techniques, we found the following sequences for the various symmetry classes of APM:

$$
\text { Type } 1,2: \quad \text { SAPM : } \quad 1,3,13,85,861
$$


TCAPM : $\quad 1,2,6,28,204$

Type 3: SAPM : $1,3,15,135,2223$

Type $4: \quad$ SAPM : $1,3,27,639$

HTAPM : 1, 1, 7, 53

We remark that the first 5 TCAPMs of type 1,2 are enumerated by the $q=2$ q-Bell numbers $B_{n}(q)$ defined recursively by $B_{0}(q)=1$ and $B_{n+1}(q)=\sum_{k=0}^{n}\left(\begin{array}{l}n \\ k\end{array}\right)_{q} B_{k}(q)$ where $\left(\begin{array}{l}n \\ k\end{array}\right)_{q}=\prod_{i=1}^{k}\left(1-q^{n+1-i}\right) /\left(1-q^{i}\right)$ is the q-binomial coefficient. It is tempting to make a conjecture, but clearly some extra numerical effort is needed here.

\subsection{Arctic phenomenon}

To conclude, we expect, at least for the case of $20 \mathrm{~V}$ with $\mathrm{DWBC} 1,2$, the existence of an "arctic phenomenon" similar to that observed for ASMs [CP10, CPS19, CS16] as well as the more general 6V-DWBC [CNP11, CPZJ10]. This was our original motivation for considering the $20 \mathrm{~V}$ model with DWBC, and will be the subject of future work.

\section{A The passage from Eq. (3.9) to Eq. (5.2) in the homogeneous case}

We wish to get an expression for (3.9) when $z_{i} \rightarrow z$ and $w_{j} \rightarrow w$ for all $i$ and $j$. Following [BDFZJ12], we start with the following identity, valid for any power series $f(z, w)$ :

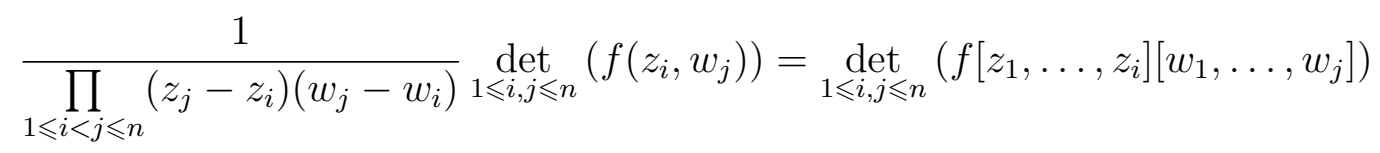

where

$$
f\left[z_{1}, \ldots, z_{i}\right]\left[w_{1}, \ldots, w_{j}\right]:=\sum_{k=1}^{i} \sum_{l=1}^{j} \frac{f\left(z_{k}, w_{l}\right)}{\prod_{\substack{k^{\prime}=1 \\ k^{\prime} \neq k}}^{i}\left(z_{k}-z_{k^{\prime}}\right) \prod_{\substack{l^{\prime}=1 \\ l^{\prime} \neq l}}^{j}\left(w_{l}-w_{l^{\prime}}\right)} .
$$

Indeed, introducing the lower triangular matrix

$$
L\left[z_{1}, \ldots, z_{n}\right]_{i, j}=\left\{\begin{array}{cc}
\prod_{\substack{k=1 \\
k \neq j}}^{i} \frac{1}{z_{j}-z_{k}} & \text { if } i \geqslant j \\
0 & \text { if } i<j
\end{array}\right.
$$

and the matrix $H$ with elements $H_{i, j}=f\left(z_{i}, w_{j}\right)$, the left hand side of (A.1) is nothing but the product $\operatorname{det}\left(L\left[z_{1}, \ldots, z_{n}\right]\right) \operatorname{det}\left(L\left[w_{1}, \ldots, w_{n}\right]^{t}\right) \operatorname{det}(H)$ while the right hand side is nothing but $\left.\operatorname{det}\left(L\left[z_{1}, \ldots, z_{n}\right]\right) \cdot H \cdot L\left[w_{1}, \ldots, w_{n}\right]^{t}\right)$ so that the identity follows immediately. 
We may now evaluate

$$
\begin{aligned}
f\left[z_{1}, \ldots, z_{i}\right]\left[w_{1}, \ldots, w_{j}\right] & =\left(\frac{1}{2 \mathrm{i} \pi}\right)^{2} \oint d t \oint d t^{\prime} \frac{1}{\prod_{k=1}^{i}\left(t-z_{k}\right)} \frac{1}{\prod_{l=1}^{j}\left(t^{\prime}-z_{l}\right)} f\left(t, t^{\prime}\right) \\
& =\sum_{m, m^{\prime}=0}^{\infty} f_{m, m^{\prime}}\left(\frac{1}{2 \mathrm{i} \pi}\right)^{2} \oint d t \oint d t^{\prime} \frac{1}{\prod_{k=1}^{i}\left(t-z_{k}\right)} \frac{1}{\prod_{l=1}^{j}\left(t^{\prime}-z_{l}\right)} t^{m} t^{\prime m^{\prime}} \\
& =\sum_{m, m^{\prime}=0}^{\infty} f_{m, m^{\prime}} \operatorname{Res}_{\infty} \frac{t^{m}}{\prod_{k=1}^{i}\left(t-z_{k}\right)} \operatorname{Res}_{\infty} \frac{t^{m^{\prime}}}{\prod_{l=1}^{j}\left(t-w_{l}\right)}
\end{aligned}
$$

where we introduced the coefficient $f_{m, m^{\prime}}$ of the series expansion $f(z, w)=\sum_{m, m^{\prime}=0}^{\infty} f_{m, m^{\prime}} z^{m} w^{m^{\prime}}$ and where the contours in the integrals were deformed so as to pick the residue at infinity. Clearly this residue is non-zero only if $m \geqslant i-1$ (respectively $m^{\prime} \geqslant j-1$ ) with the result

$$
\operatorname{Res}_{\infty} \frac{t^{m}}{\prod_{k=1}^{i}\left(t-z_{k}\right)}=\sum_{\substack{p_{1}, p_{2}, \ldots, p_{i} \geqslant 0 \\ \sum_{k=1}^{i} p_{k}=m+1-i}} \prod_{k=1}^{i} z_{k}^{p_{k}}=h_{m+1-i}\left(z_{1}, \ldots, z_{i}\right)
$$

in terms of the complete symmetric polynomial $h_{m}$. This yields

$$
f\left[z_{1}, \ldots, z_{i}\right]\left[w_{1}, \ldots, w_{j}\right]=\sum_{\substack{m \geqslant i-1 \\ m^{\prime} \geqslant j-1}} f_{m, m^{\prime}} h_{m+1-i}\left(z_{1}, \ldots, z_{i}\right) h_{m^{\prime}+1-j}\left(w_{1}, \ldots, w_{j}\right)
$$

and in particular

$$
\begin{aligned}
f[\underbrace{z, \ldots, z}_{i}][\underbrace{w, \ldots, w}_{j}]= & \sum_{\substack{m \geqslant i-1 \\
m^{\prime} \geqslant j-1}} f_{m, m^{\prime}} h_{m+1-i}(\underbrace{z, \ldots, z}_{i}) h_{m^{\prime}+1-j}(\underbrace{w, \ldots, w}_{j}) \\
& =\sum_{\substack{m \geqslant i-1 \\
m^{\prime} \geqslant j-1}} f_{m, m^{\prime}}\left(\begin{array}{c}
m \\
i-1
\end{array}\right) z^{m+1-i}\left(\begin{array}{c}
m^{\prime} \\
j-1
\end{array}\right) w^{m^{\prime}+1-j} \\
& =\left.f(z+r, w+s)\right|_{r^{i-1} s^{j-1}} .
\end{aligned}
$$

This leads to the desired limit

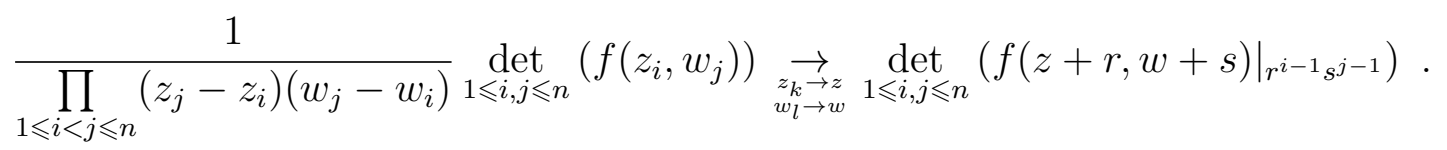

Using

$$
\frac{1}{a(i, j) b(i, j)}=\frac{1}{z_{i}-w_{j}} \frac{1}{q^{-2} z_{i}-q^{2} w_{j}}=\frac{q^{2}}{\left(1-q^{4}\right) w_{j}}\left(\frac{1}{z_{i}-w_{j}}-\frac{1}{z_{i}-q^{4} w_{j}}\right),
$$


we deduce

$$
\begin{aligned}
& \frac{\prod_{i=1}^{n} c(i, i) \prod_{i, j=1}^{n}(a(i, j) b(i, j))}{\prod_{1 \leqslant i<j \leqslant n}\left(z_{i}-z_{j}\right)\left(w_{j}-w_{i}\right)} \operatorname{det}_{1 \leqslant i, j \leqslant n}\left(\frac{1}{a(i, j) b(i, j)}\right) \\
& =\frac{\prod_{i=1}^{n} c(i, i) \prod_{i, j=1}^{n}(a(i, j) b(i, j)) \prod_{j=1}^{n}\left(\frac{q^{2}}{\left(1-q^{4}\right) w_{j}}\right)}{\prod_{1 \leqslant i<j \leqslant n}\left(z_{i}-z_{j}\right)\left(w_{j}-w_{i}\right)} \operatorname{det}_{1 \leqslant i, j \leqslant n}\left(\frac{1}{z_{i}-w_{j}}-\frac{1}{z_{i}-q^{4} w_{j}}\right) \\
& \underset{\substack{z_{k} \rightarrow z \\
w_{l} \rightarrow w}}{\rightarrow}(-1)^{\frac{n(n-1)}{2}} \prod_{i=1}^{n}\left(\left(q^{2}-q^{-2}\right) \sqrt{z w}\right) \prod_{i, j=1}^{n}\left((z-w)\left(q^{-2} z-q^{2} w\right)\right) \prod_{j=1}^{n} \frac{q^{2}}{\left(1-q^{4}\right) w} \\
& \times \operatorname{det}_{1 \leqslant i, j \leqslant n}\left(\left.\left(\frac{1}{(z+r)-(w+s)}-\frac{1}{(z+r)-q^{4}(w+s)}\right)\right|_{r^{i-1} s^{j-1}}\right) .
\end{aligned}
$$

This is nothing but (5.2).

\section{B A proof of Eq. (5.5)}

We now wish to get an expression for (3.9) when $z_{i} \rightarrow z, i=1, \ldots, n, w_{j} \rightarrow w, j=$ $1, \ldots n-1$ and $w_{n} \rightarrow w u$. Following again [BDFZJ12], we have the following general identity:

$$
\frac{1}{\prod_{1 \leqslant i<j \leqslant n}\left(z_{j}-z_{i}\right) \prod_{1 \leqslant i<j \leqslant n-1}\left(w_{j}-w_{i}\right)} \operatorname{det}_{1 \leqslant i, j \leqslant n}\left(f\left(z_{i}, w_{j}\right)\right)=\operatorname{det}_{1 \leqslant i, j \leqslant n}\left(\begin{array}{cc}
f\left[z_{1}, \ldots, z_{i}\right]\left[w_{1}, \ldots, w_{j}\right] & j \leqslant n-1 \\
f\left[z_{1}, \ldots, z_{i}\right]\left[w_{n}\right] & j=n
\end{array}\right)
$$

where $f\left[z_{1}, \ldots, z_{i}\right]\left[w_{1}, \ldots, w_{j}\right]$ is as in (A.2). This identity is obtained as in Appendix A by multiplying the matrix $H$ with elements $H_{i, j}=f\left(z_{i}, w_{j}\right)$ to the left by the matrix $L\left[z_{1}, \ldots, z_{n}\right]$ of (A.3) and to the right by the transpose of the matrix $L^{(n)}\left[w_{1}, \ldots, w_{n-1}\right]$ with elements

$$
L^{(n)}\left[w_{1}, \ldots, w_{n-1}\right]_{i, j}=\left\{\begin{array}{cc}
\prod_{k=1}^{i} \frac{1}{w_{j}-w_{k}} & \text { if } n-1 \geqslant i \geqslant j \\
k \neq j & \text { if } i=n \\
\delta_{n, j} & \text { if } i<j \\
0 &
\end{array}\right.
$$

then taking the determinant. We have in particular in the desired limit

$$
\begin{aligned}
\prod_{1 \leqslant i<j \leqslant n}\left(z_{j}-z_{i}\right) \prod_{1 \leqslant i<j \leqslant n-1}\left(w_{j}-w_{i}\right) & \operatorname{det}_{1 \leqslant i, j \leqslant n}\left(f\left(z_{i}, w_{j}\right)\right) \\
& \operatorname{det}_{\substack{z_{k} \rightarrow z, w_{l<n} \rightarrow w \\
w_{n \rightarrow w u} \rightarrow w_{1 \leqslant i, j \leqslant n}}}\left(\begin{array}{cc}
\left.f(z+r, w+s)\right|_{r^{i-1} s^{j-1}} & j \leqslant n-1 \\
\left.f(z+r, w u)\right|_{r^{i-1}} & j=n
\end{array}\right)
\end{aligned}
$$


We now deduce

$$
\begin{aligned}
& \frac{\prod_{i=1}^{n} c(i, i) \prod_{i, j=1}^{n}(a(i, j) b(i, j))}{\prod_{1 \leqslant i<j \leqslant n}\left(z_{i}-z_{j}\right)\left(w_{j}-w_{i}\right)} \operatorname{det}_{1 \leqslant i, j \leqslant n}\left(\frac{1}{a(i, j) b(i, j)}\right) \\
& =\prod_{i=1}^{n-1} c(i, i) \prod_{i=1}^{n} \prod_{j=1}^{n-1}(a(i, j) b(i, j)) \prod_{j=1}^{n-1}\left(\frac{q^{2}}{\left(1-q^{4}\right) w_{j}}\right) \\
& \times c(n, n) \prod_{i=1}^{n}(a(i, n) b(i, n))\left(\frac{q^{2}}{\left(1-q^{4}\right) w_{n}}\right) \frac{1}{\prod_{i=1}^{n-1}\left(w_{n}-w_{i}\right)} \\
& \times \frac{1}{\prod_{1 \leqslant i<j \leqslant n}\left(z_{i}-z_{j}\right) \prod_{1 \leqslant i<j \leqslant n-1}\left(w_{j}-w_{i}\right)} \operatorname{det}_{1 \leqslant i, j \leqslant n}\left(\frac{1}{z_{i}-w_{j}}-\frac{1}{z_{i}-q^{4} w_{j}}\right) \\
& \underset{\substack { z_{k} \rightarrow z \\
\begin{subarray}{c}{w_{n} \rightarrow w u \\
w_{n} \rightarrow \infty{ z _ { k } \rightarrow z \\
\begin{subarray} { c } { w _ { n } \rightarrow w u \\
w _ { n } \rightarrow \infty } }\end{subarray}}{\rightarrow}(-1)^{\frac{n(n-1)}{2}} \prod_{i=1}^{n-1}\left(\left(q^{2}-q^{-2}\right) \sqrt{z w}\right) \prod_{i=1}^{n} \prod_{j=1}^{n-1}\left((z-w)\left(q^{-2} z-q^{2} w\right)\right) \prod_{j=1}^{n-1} \frac{q^{2}}{\left(1-q^{4}\right) w} \\
& \times\left(q^{2}-q^{-2}\right) \sqrt{z w u} \prod_{i=1}^{n}\left((z-w u)\left(q^{-2} z-q^{2} w u\right)\right) \frac{q^{2}}{\left(1-q^{4}\right) w u} \frac{1}{\prod_{i=1}^{n-1}(w u-w)} \\
& \times \operatorname{det}_{1 \leqslant i, j \leqslant n}\left(\begin{array}{cc}
\left.\left(\frac{1}{(z+r)-(w+s)}-\frac{1}{(z+r)-q^{4}(w+s)}\right)\right|_{r^{i-1} s^{j-1}} & j \leqslant n-1 \\
\left.\left(\frac{1}{(z+r)-w u}-\frac{1}{(z+r)-q^{4} w u}\right)\right|_{r^{i-1}} & j=n
\end{array}\right) .
\end{aligned}
$$

This is nothing but (5.5).

\section{References}

[And80] George E. Andrews, Macdonald's conjecture and descending plane partitions, Combinatorics, representation theory and statistical methods in groups, Lecture Notes in Pure and Appl. Math., vol. 57, Dekker, New York, 1980, pp. 91106. MR 588196

[Bax89] Rodney J. Baxter, Exactly solved models in statistical mechanics, Academic Press, Inc. [Harcourt Brace Jovanovich, Publishers], London, 1989, Reprint of the 1982 original. MR 998375

[BDFZJ12] Roger E. Behrend, Philippe Di Francesco, and Paul Zinn-Justin, On the weighted enumeration of alternating sign matrices and descending plane partitions, J. Combin. Theory Ser. A 119 (2012), no. 2, 331-363. MR 2860598

[BDFZJ13] _ A doubly-refined enumeration of alternating sign matrices and descending plane partitions, J. Combin. Theory Ser. A 120 (2013), no. 2, 409432. MR 2995049 
[BF06] Pavel M. Bleher and Vladimir V. Fokin, Exact solution of the six-vertex model with domain wall boundary conditions. disordered phase, Communications in Mathematical Physics 268 (2006), no. 1, 223-284

[BK18] Alexey Bufetov and Alisa Knizel, Asymptotics of random domino tilings of rectangular aztec diamonds, Ann. Inst. H. Poincaré Probab. Statist. 54 (2018), no. 3, 1250-1290

[Bre99] David M. Bressoud, Proofs and confirmations, MAA Spectrum, Mathematical Association of America, Washington, DC; Cambridge University Press, Cambridge, 1999, The story of the alternating sign matrix conjecture. MR 1718370

[CEP96] Henry Cohn, Noam Elkies, and James Propp, Local statistics for random domino tilings of the aztec diamond, Duke Math. J. 85 (1996), no. 1, arXiv:math/0008243 [math.CO].

[Che92] Ivan Cherednik, Quantum Knizhnik-Zamolodchikov equations and affine root systems, Comm. Math. Phys. 150 (1992), no. 1, 109-136. MR 1188499

[Ciu97] Mihai Ciucu, Enumeration of perfect matchings in graphs with reflective symmetry, J. Combin. Theory Ser. A 77 (1997), no. 1, 67-97. MR 1426739

[CNP11] Filippo Colomo, Vanni Noferini, and Andrei G. Pronko, Algebraic arctic curves in the domain-wall six-vertex model, J. Phys. A: Math. Theor. 44 (2011), no. 19, 195201, arXiv:1012. 2555 [math-ph].

[CP10] Filippo Colomo and Andrei G. Pronko, The limit shape of large alternating sign matrices, SIAM J. Discrete Math. 24 (2010), no. 4, 1558-1571, arXiv:0803.2697 [math-ph].

[CPS19] Filippo Colomo, Andrei G. Pronko, and Andrea Sportiello, Arctic curve of the free-fermion six-vertex model in an L-shaped domain, Journal of Statistical Physics 174 (2019), no. 1, 1-27

[CPZJ10] Filippo Colomo, Andrei G. Pronko, and Paul Zinn-Justin, The arctic curve of the domain wall six-vertex model in its antiferroelectric regime, J. Stat. Mech. Theory Exp. (2010), no. 3, L03002, 11. MR 2629602

[CS11] Luigi Cantini and Andrea Sportiello, Proof of the Razumov-Stroganov conjecture, J. Combin. Theory Ser. A 118 (2011), no. 5, 1549-1574. MR 2771600

[CS16] Filippo Colomo and Andrea Sportiello, Arctic curves of the six-vertex model on generic domains: the tangent method, J. Stat. Phys. 164 (2016), no. 6, 1488-1523, arXiv: 1605.01388 [math-ph].

[DFG19] Philippe Di Francesco and Emmanuel Guitter, The arctic curve for aztec rectangles with defects via the tangent method, J. Stat. Phys. (2019), https://doi.org/10.1007/s10955-019-02315-2, arXiv:1902.06478 [math-ph]. 
[DFZJ05a] Philippe Di Francesco and Paul Zinn-Justin, Around the Razumov-Stroganov conjecture: proof of a multi-parameter sum rule, Electron. J. Combin. 12 (2005), Research Paper 6, 27. MR 2134169

[DFZJ05b] _ The quantum Knizhnik-Zamolodchikov equation, generalized Razumov-Stroganov sum rules and extended Joseph polynomials, J. Phys. A 38 (2005), no. 48, L815-L822. MR 2185933

$\left[\mathrm{FMP}^{+} 15\right]$ Laura Florescu, Daniela Morar, David Perkinson, Nick Salter, and Tianyuan $\mathrm{Xu}$, Sandpiles and dominos, Electron. J. Combin. 22 (2015), no. 1, Paper 1.66, 35. MR 3336580

[GAL72] M. Lauwrence Glasser, Douglas B. Abraham, and Eliott H. Lieb, Analytic properties of the free energy for the "ice" models, J. Mathematical Phys. 13 (1972), 887-900. MR 0434259

[GV85] Ira Gessel and Gérard Viennot, Binomial determinants, paths, and hook length formulae, Adv. Math. 58 (1985), no. 3, 300-321

[ICK92] Anatoli G. Izergin, David A. Coker, and Vladimir E. Korepin, Determinant formula for the six-vertex model, J. Phys. A 25 (1992), no. 16, 4315-4334. MR 1181591

[Ize87] Anatoli G. Izergin, Partition function of a six-vertex model in a finite volume, Dokl. Akad. Nauk SSSR 297 (1987), no. 2, 331-333. MR 919260

[Kas63] Pieter W. Kasteleyn, Dimer statistics and phase transitions, J. Mathematical Phys. 4 (1963), 287-293. MR 0153427

[Kel74] Stewart B. Kelland, Twenty-vertex model on a triangular lattice, Australian Journal of Physics 27 (1974), 813

[Kor82] Vladimir E. Korepin, Calculation of norms of bethe wave functions, Commun. Math. Phys. 86 (1982), no. 3, 391-418

[Kra06] Christian Krattenthaler, Descending plane partitions and rhombus tilings of a hexagon with a triangular hole, European J. Combin. 27 (2006), no. 7, 1138-1146. MR 2259946

[Kup96] Greg Kuperberg, Another proof of the alternative-sign matrix conjecture, Int. Math. Res. Not., 1996 (1996), no. 3, 139-150, https://doi.org/10.1155/S1073792896000128.

[Kup02] _ Symmetry classes of alternating-sign matrices under one roof, Ann. Math. (2002), 835-866, arXiv:math/0008184 [math.CO].

[Lal03] Pierre Lalonde, Lattice paths and the antiautomorphism of the poset of descending plane partitions, Discrete Math. 271 (2003), no. 1-3, 311-319. MR 1999553

[Lin73] Bernt Lindström, On the vector representations of induced matroids, Bull. London Math. Soc. 5 (1973), no. 1, 85-90 
[MRR83] William H. Mills, David P. Robbins, and Howard Rumsey, Alternating sign matrices and descending plane partitions, J. Combin. Theory Ser. A 34 (1983), no. 3, 340-359

[MRR86] William H. Mills, David P. Robbins, and Howard Rumsey, Jr., Selfcomplementary totally symmetric plane partitions, J. Combin. Theory Ser. A 42 (1986), no. 2, 277-292. MR 847558

[Pac97] Lior Pachter, Combinatorial approaches and conjectures for 2-divisibility problems concerning domino tilings of polyominoes, Electron. J. Combin. 4 (1997), no. 1, Research Paper 29, 10. MR 1479755

[RS04] Aleksandr Vital'evich Razumov and Yu G. Stroganov, Refined enumerations of some symmetry classes of alternating-sign matrices, Theor. Math. Phys. 141 (2004), no. 3, 1609-1630, arXiv:math-ph/0312071

[TF61] Harold N. V. Temperley and Michael E. Fisher, Dimer problem in statistical mechanics - an exact result, Philos. Mag. (8) 6 (1961), 1061-1063. MR 0136398

[Zei96a] Doron Zeilberger, Proof of the alternating sign matrix conjecture, Electron. J. Combin. 3 (1996), no. 2, R13, arXiv:math/9407211 [math.CO].

[Zei96b] _ Proof of the refined alternating sign matrix conjecture, New York J. Math. 2 (1996), 59-68, arXiv:math/9606224 [math.CO].

[ZJ00] Paul Zinn-Justin, Six-vertex model with domain wall boundary conditions and one-matrix model, Phys. Rev. E 62 (2000), 3411-3418 